\title{
Bond order densities in real space Supplementary Information.
}

J. L. Casals-Sainz, A. Fernández-Alarcón, E. Francisco, A. Costales and A. Martín Pendás*

Departamento de Química Física y Analítica.

Universidad de Oviedo. 33006 Oviedo. Spain. 


\section{Contents}

1 Cartesian coordinates of $\mathrm{HCN}$ and $\mathrm{CH}_{2} \mathrm{~N}_{2} \quad 2$

2 BOD maps. $\quad 2$

\section{Cartesian coordinates of $\mathrm{HCN}$ and $\mathrm{CH}_{2} \mathrm{~N}_{2}$}

All data in au.

$\mathrm{HCN}$

$\begin{array}{lrrr}\text { N } & 0.0193803074 & -0.0053429553 & 0.0000000000 \\ \text { C } & 1.1907432864 & 0.0064899042 & 0.0000000000 \\ \text { H } & 0.9487500774 & 1.1446866928 & 0.0000000000\end{array}$

$\mathrm{CH}_{2} \mathrm{~N}_{2}$

$\begin{array}{lrrr}\mathrm{C} & 0.0000000000 & 0.0000000000 & -1.2145020810 \\ \mathrm{~N} & 0.0000000000 & 0.0000000000 & 0.0729989454 \\ \mathrm{H} & 0.9526303374 & -0.0000000000 & -1.7167134007 \\ \mathrm{H} & -0.9526303374 & 0.0000000000 & -1.7167134007 \\ \mathrm{~N} & -0.0000000000 & 0.0000000000 & 1.2019699370\end{array}$

\section{BOD maps.}

In this Section, a set of Tables containing the maps of the BOD for all the bonding channels together with the occupation numbers of their corresponding natural orbitals are presented. All data are reported in atomic units. In the case of degenerate $\pi$ orbitals, only one component is shown. Heteroatomic, followed by homodiatomic molecules, in alphabetical order. 
Table S 1: Bonding channels and the corresponding occupation numbers as well as maps of bonding densities for AlO.

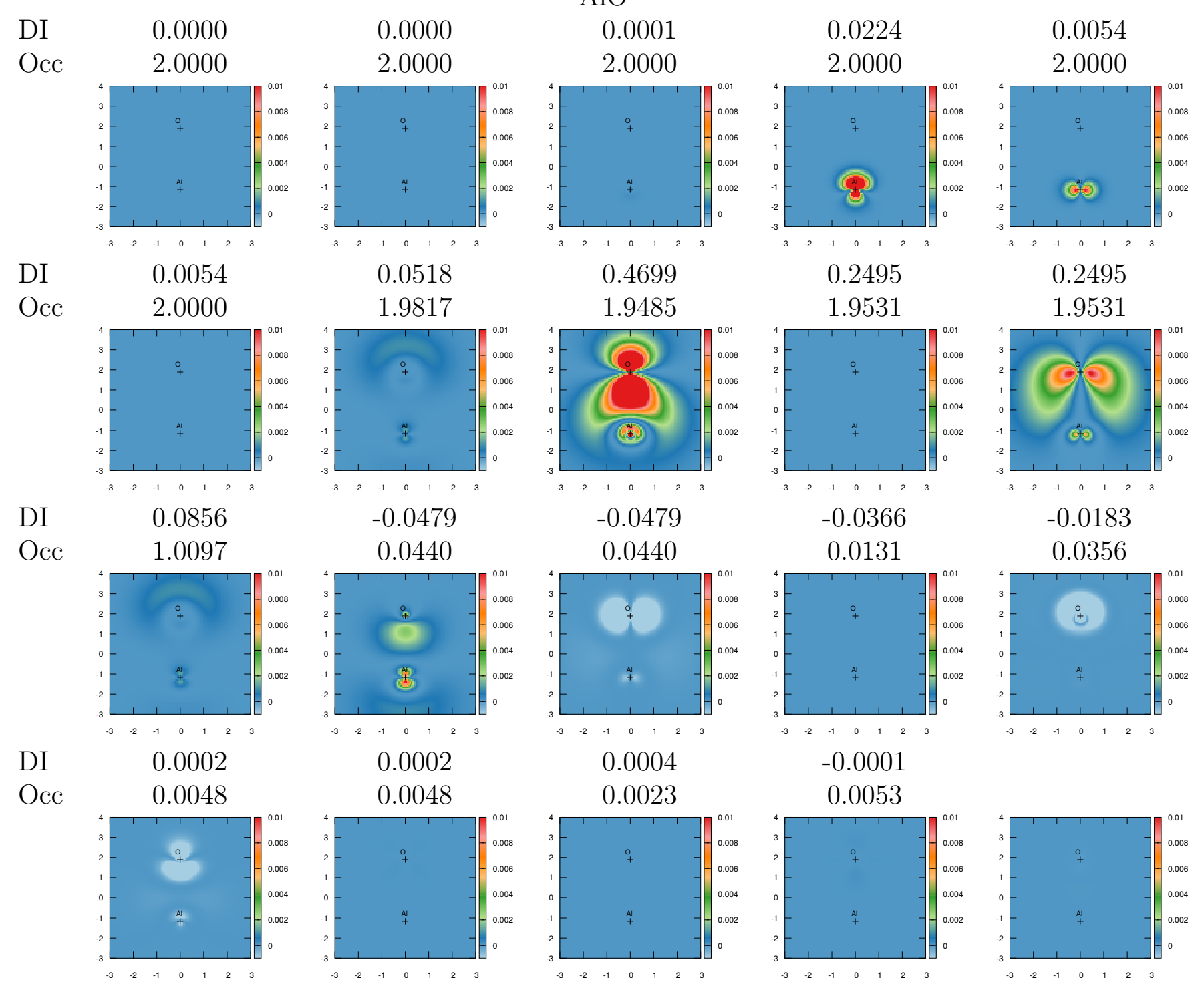


Table S 2: Bonding channels and the corresponding occupation numbers as well as maps of bonding densities for AlS.

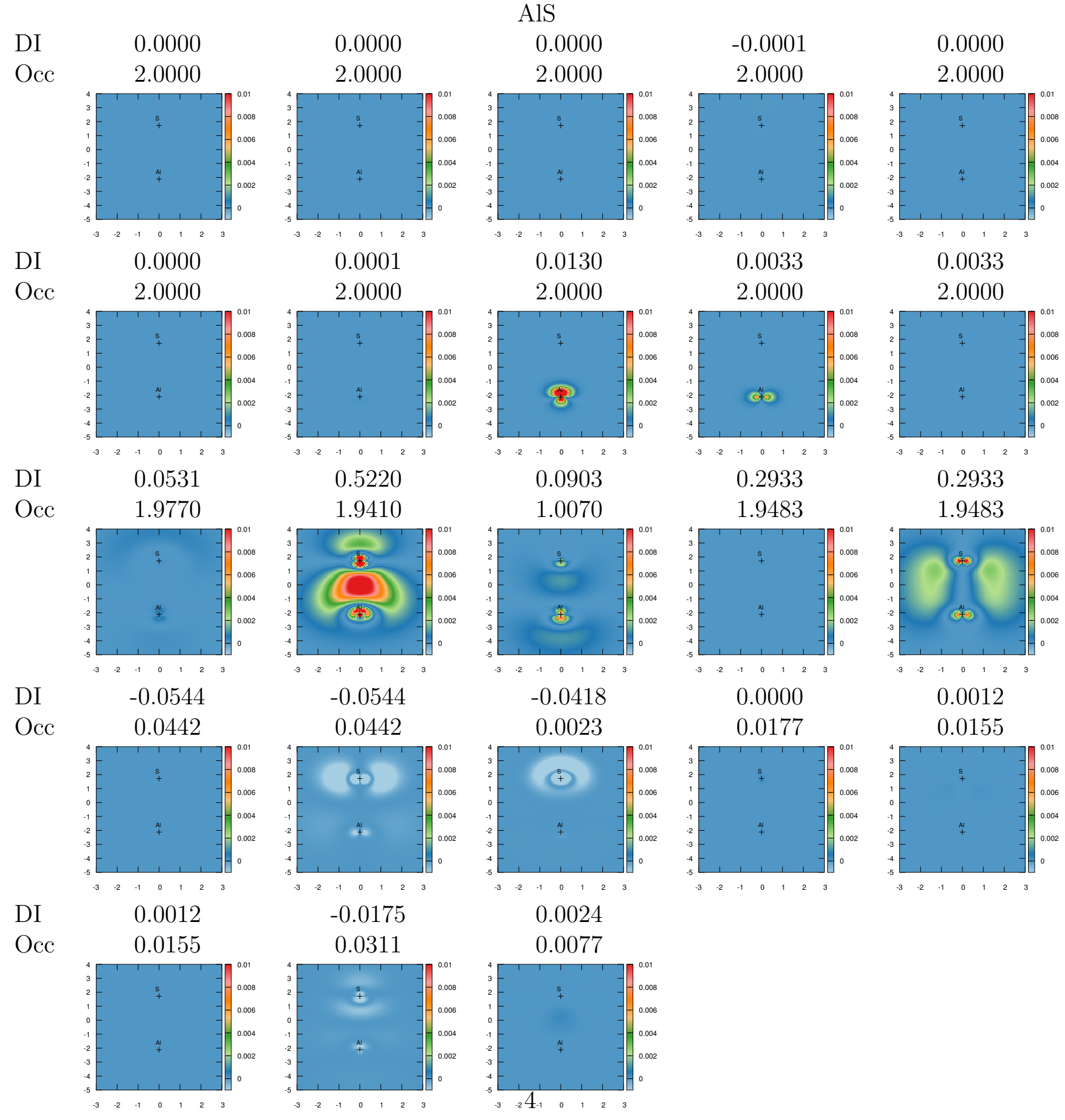


Table S 3: Bonding channels and the corresponding occupation numbers as well as maps of bonding densities for $\mathrm{BCl}$.

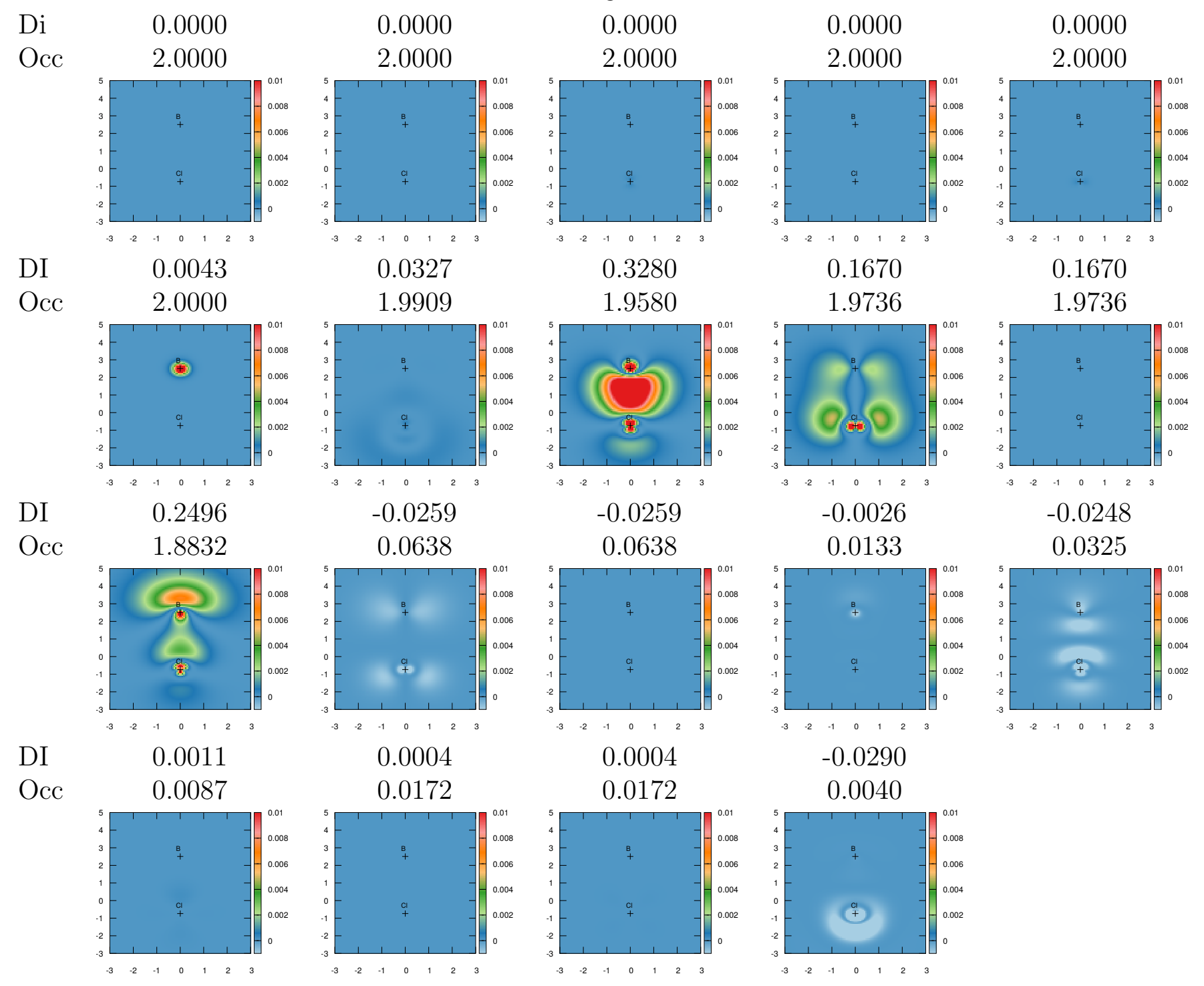


Table S 4: Bonding channels and the corresponding occupation numbers as well as maps of bonding densities for BC.

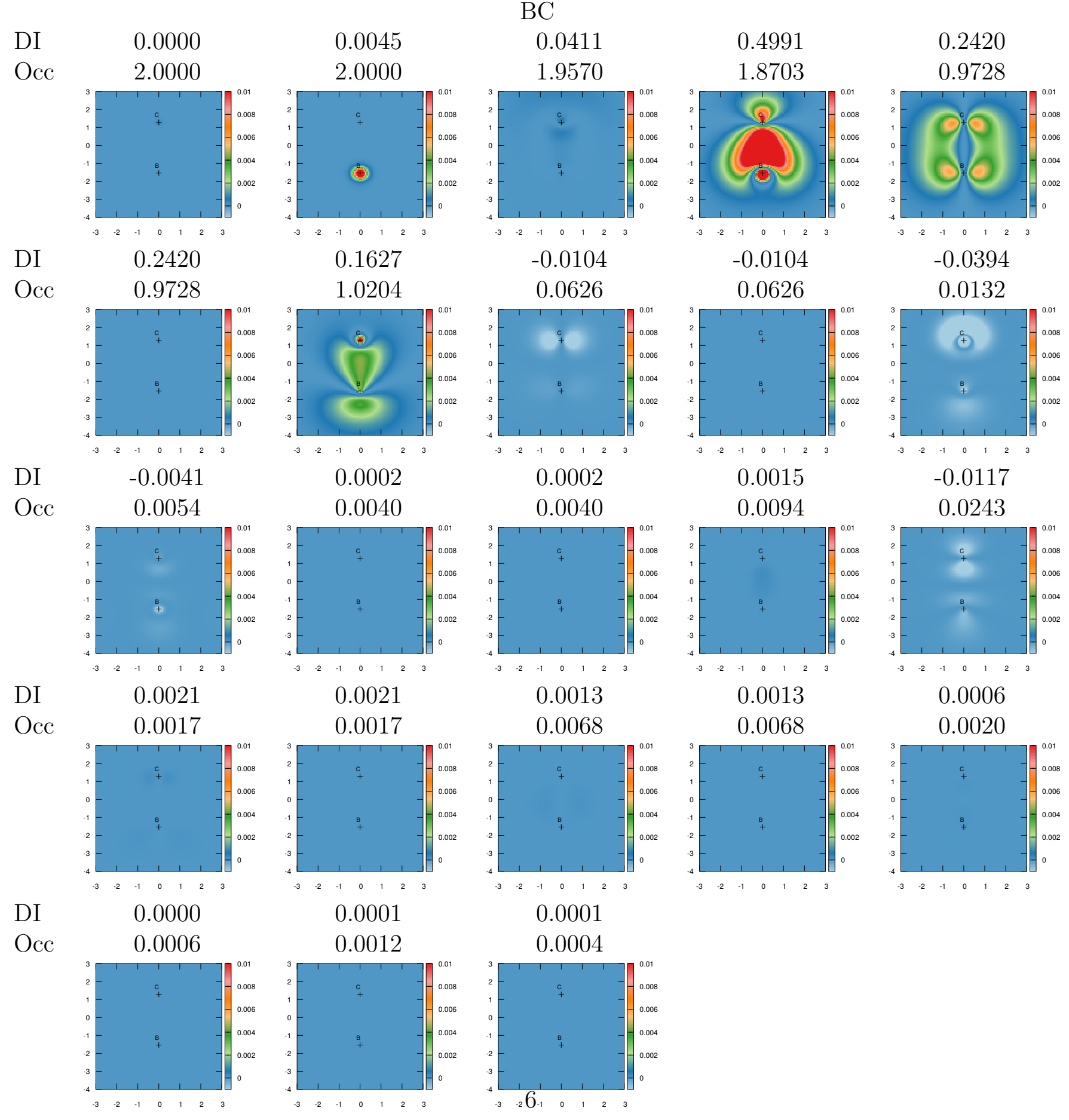


Table S 5: Bonding channels and the corresponding occupation numbers as well as maps of bonding densities for $\mathrm{BeCl}$.

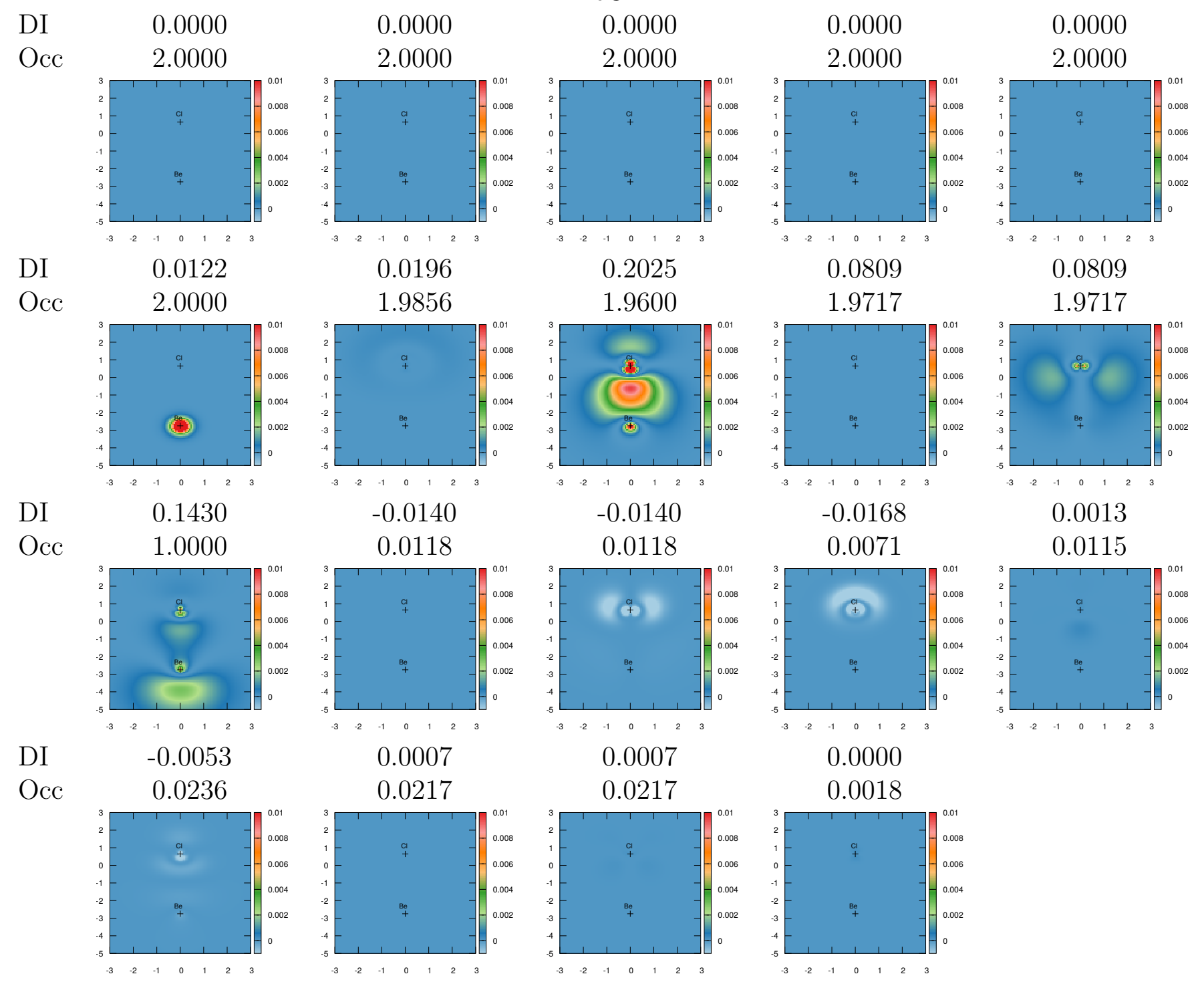


Table S 6: Bonding channels and the corresponding occupation numbers as well as maps of bonding densities for BeF.

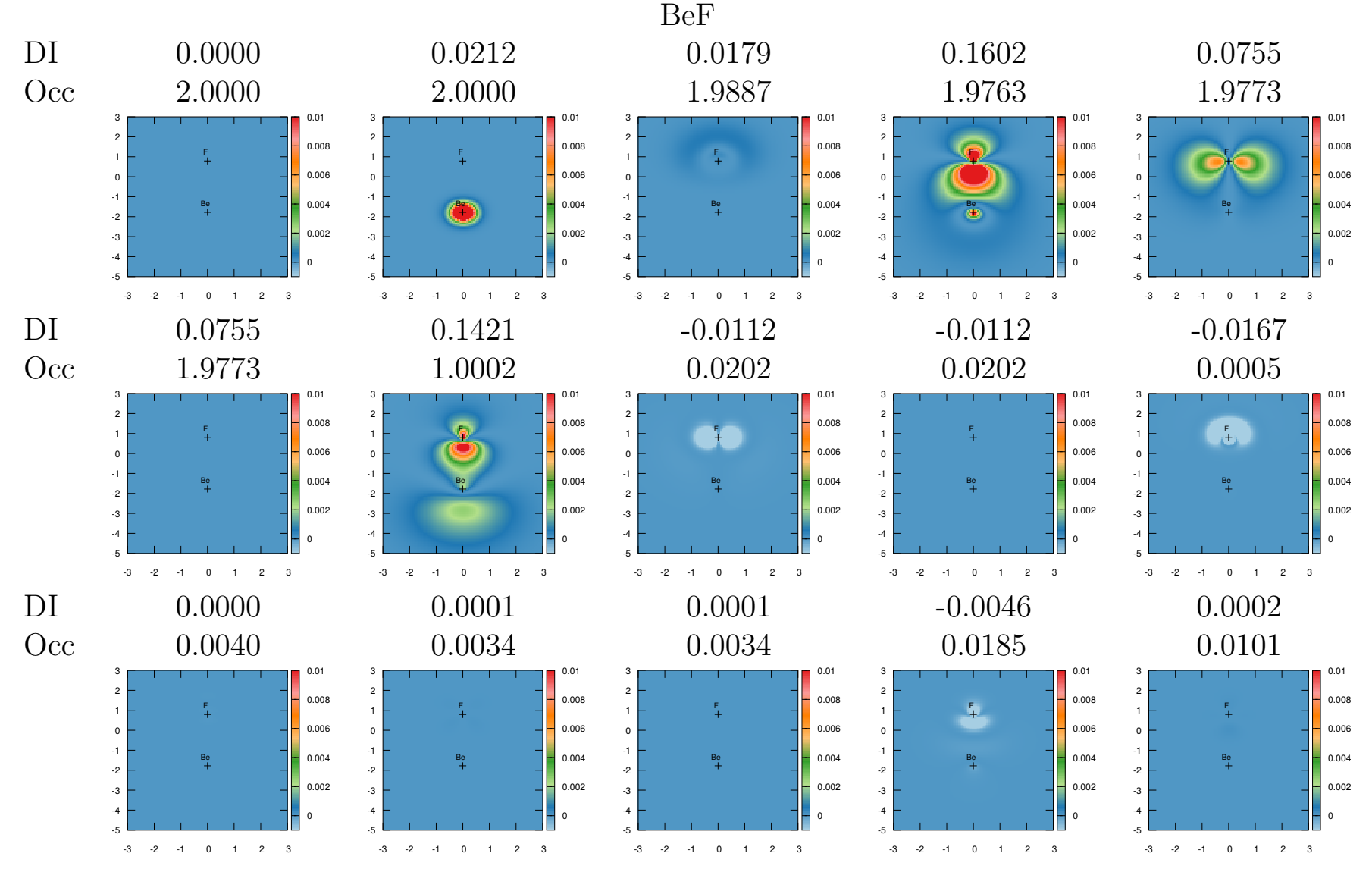


Table S 7: Bonding channels and the corresponding occupation numbers as well as maps of bonding densities for $\mathrm{BeO}$.

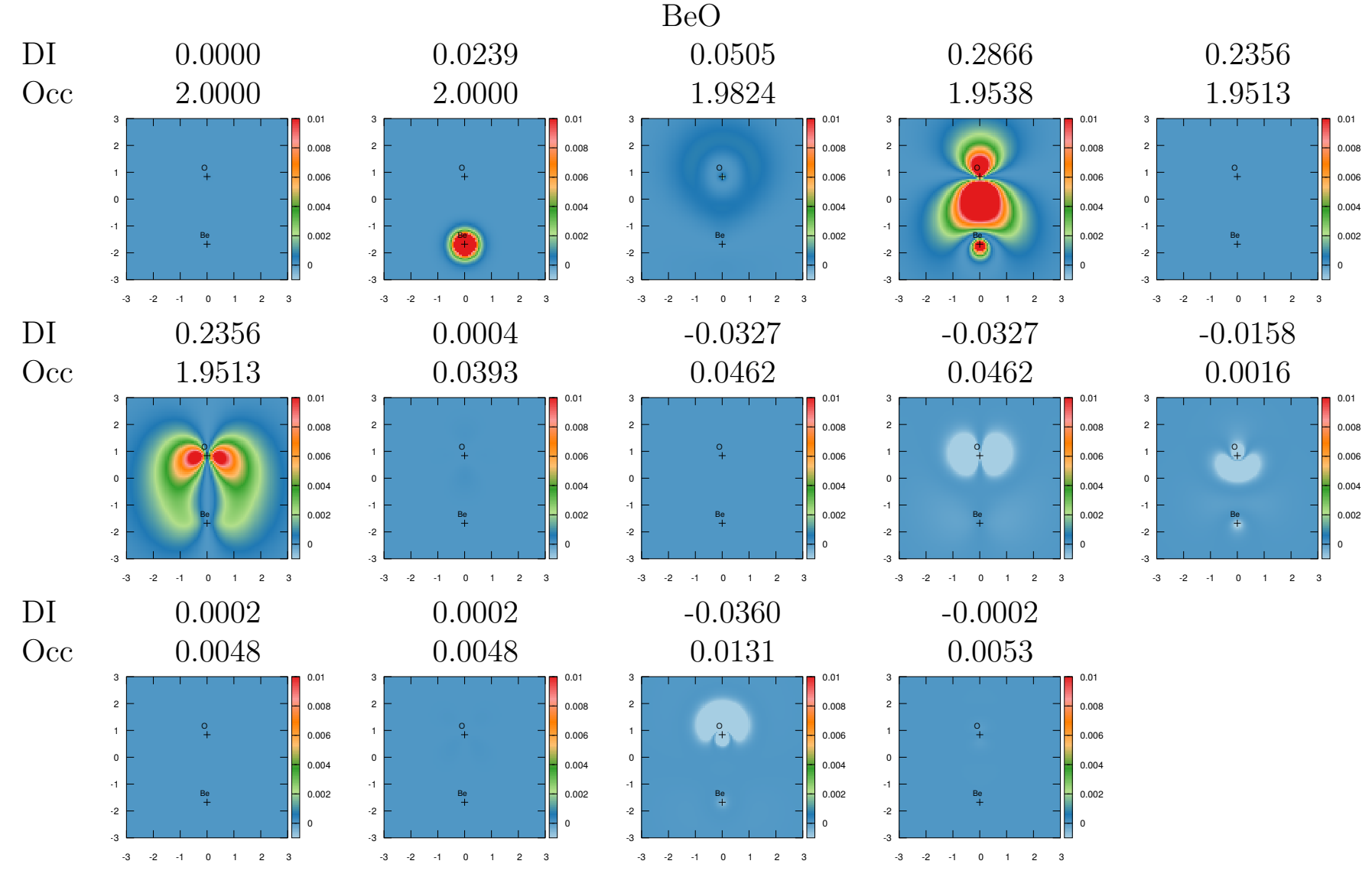


Table S 8: Bonding channels and the corresponding occupation numbers as well as maps of bonding densities for BeS.

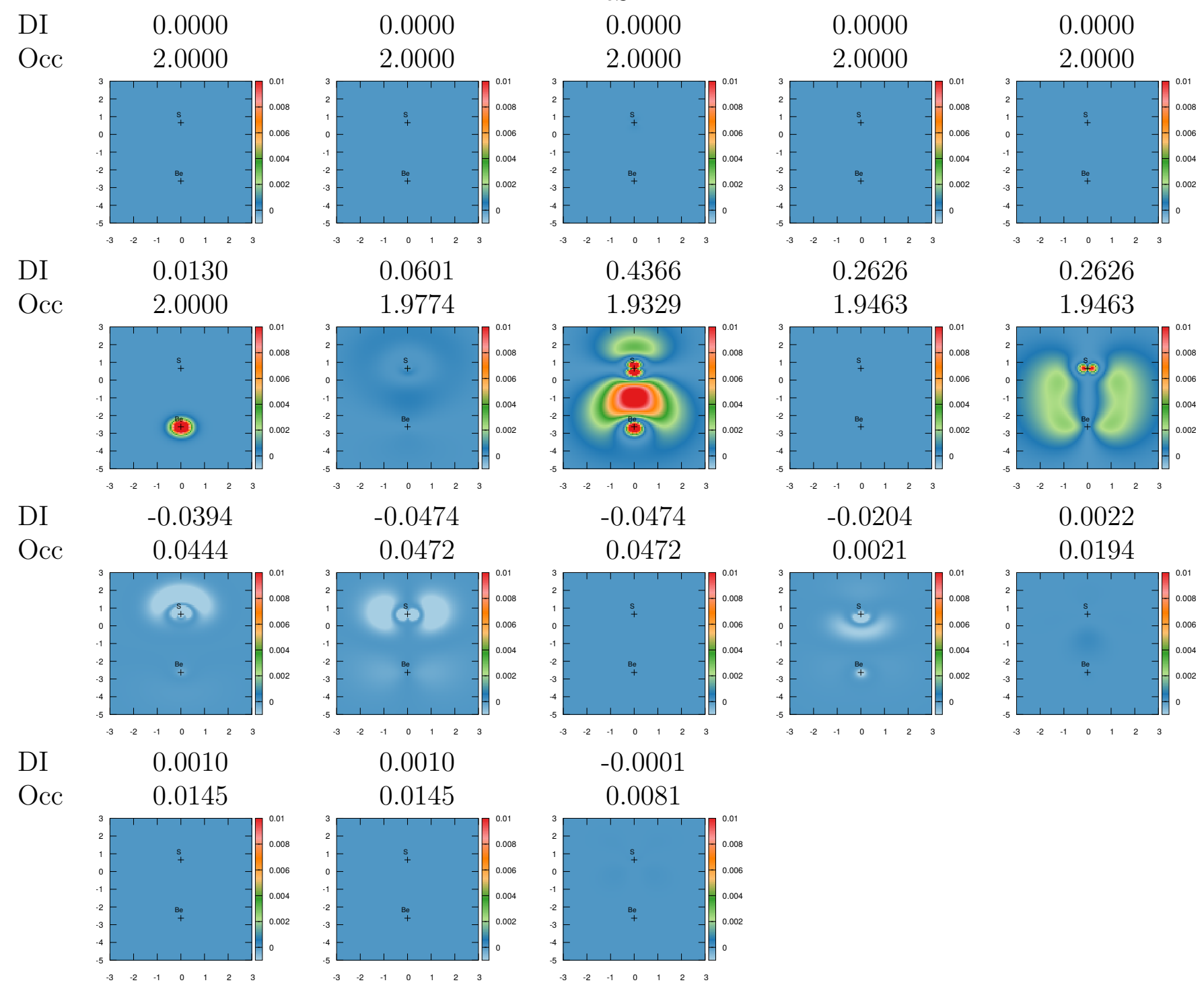


Table S 9: Bonding channels and the corresponding occupation numbers as well as maps of bonding densities for BF.

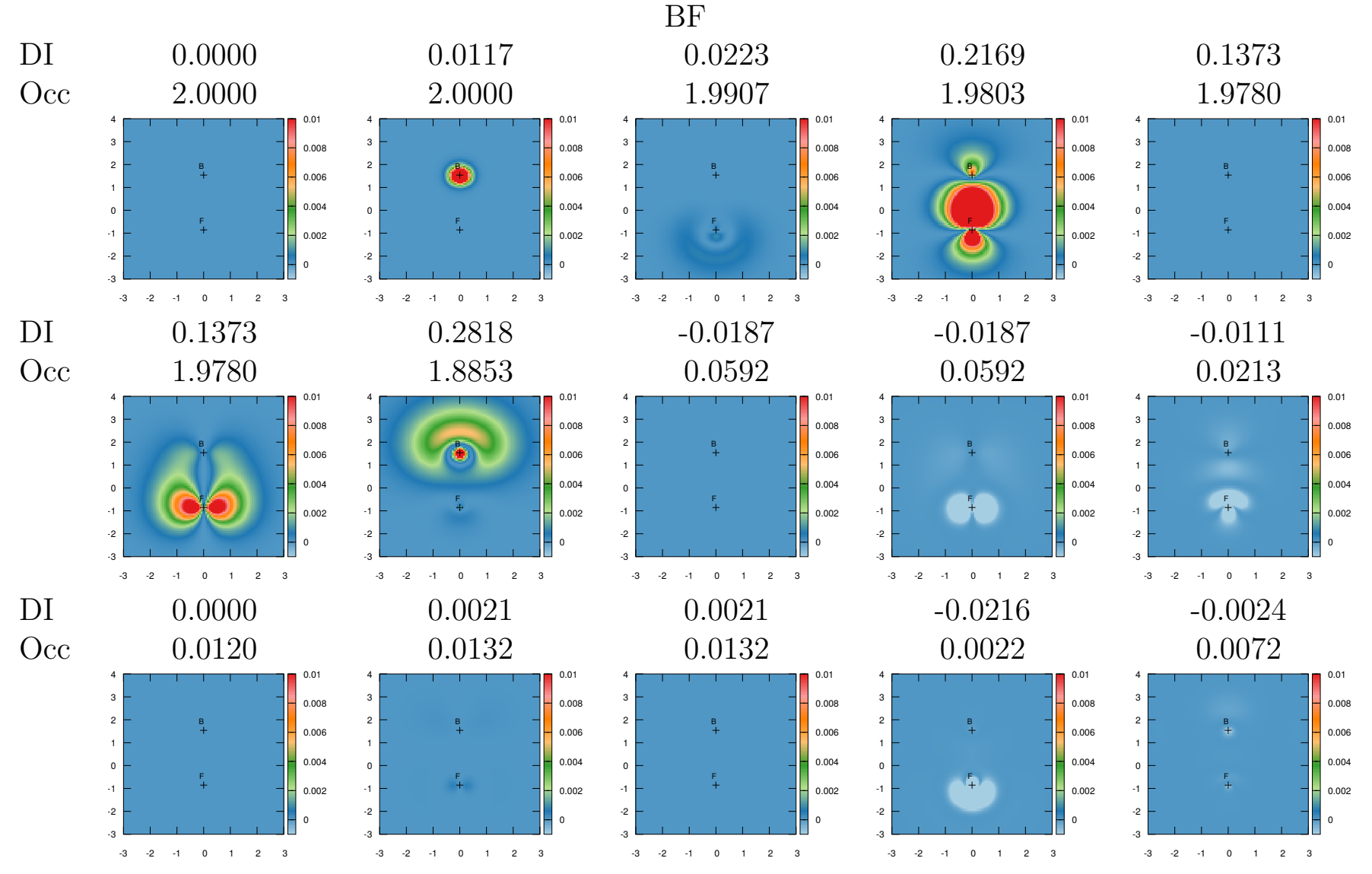


Table S 10: Bonding channels and the corresponding occupation numbers as well as maps of bonding densities for BN.

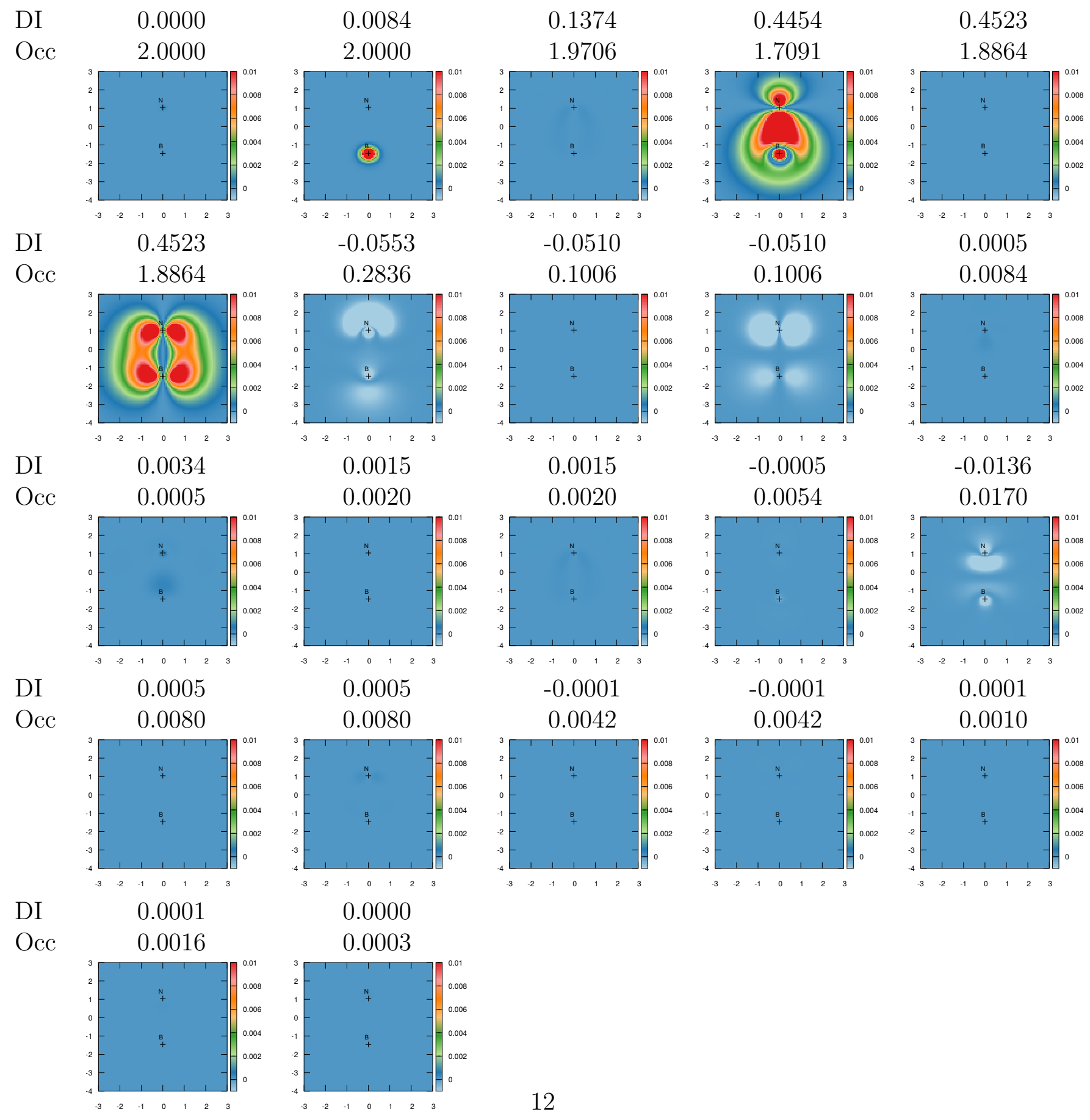


Table S 11: Bonding channels and the corresponding occupation numbers as well as maps of bonding densities for $\mathrm{BO}^{-}$.

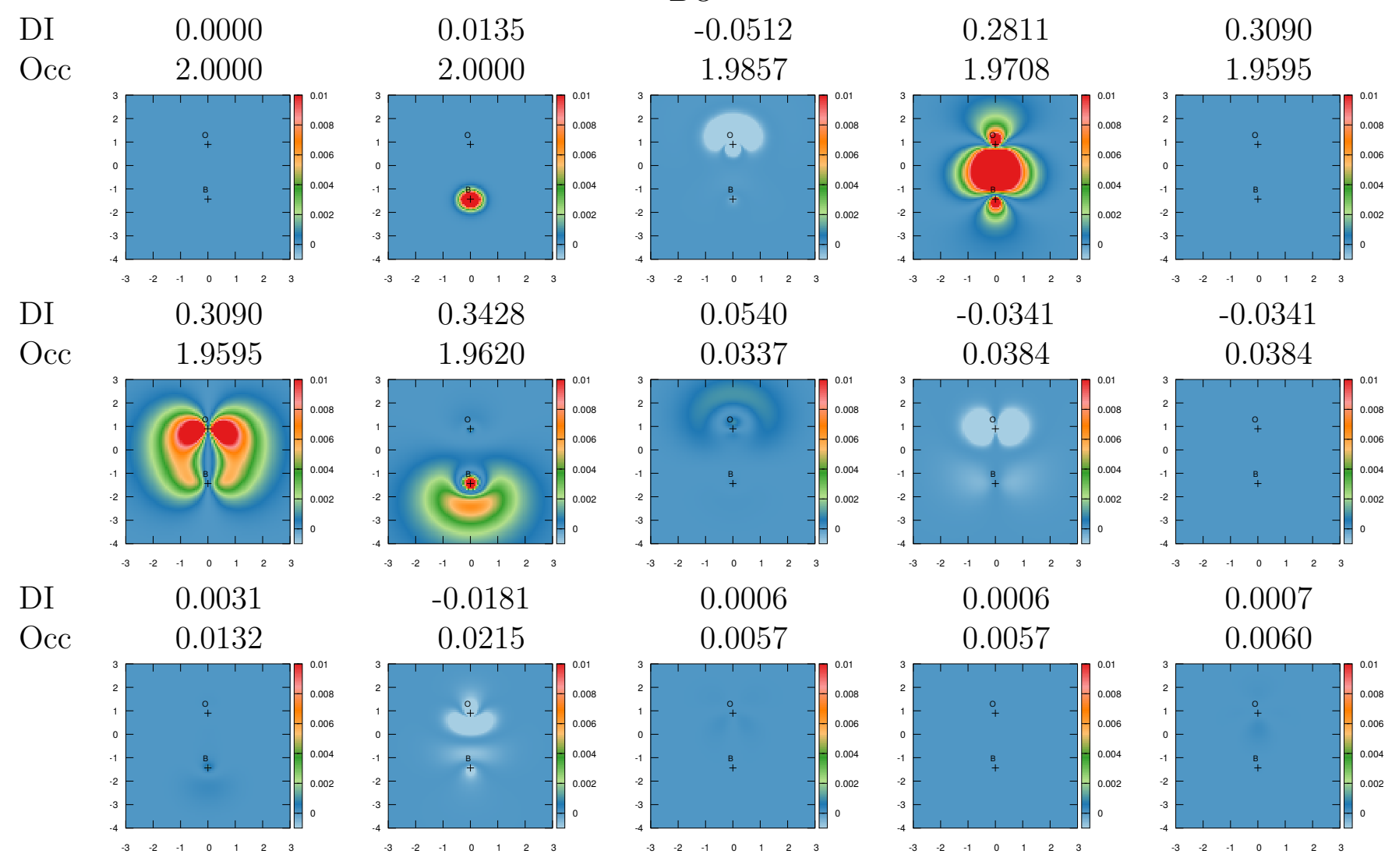


Table S 12: Bonding channels and the corresponding occupation numbers as well as maps of bonding densities for $\mathrm{BO}$.

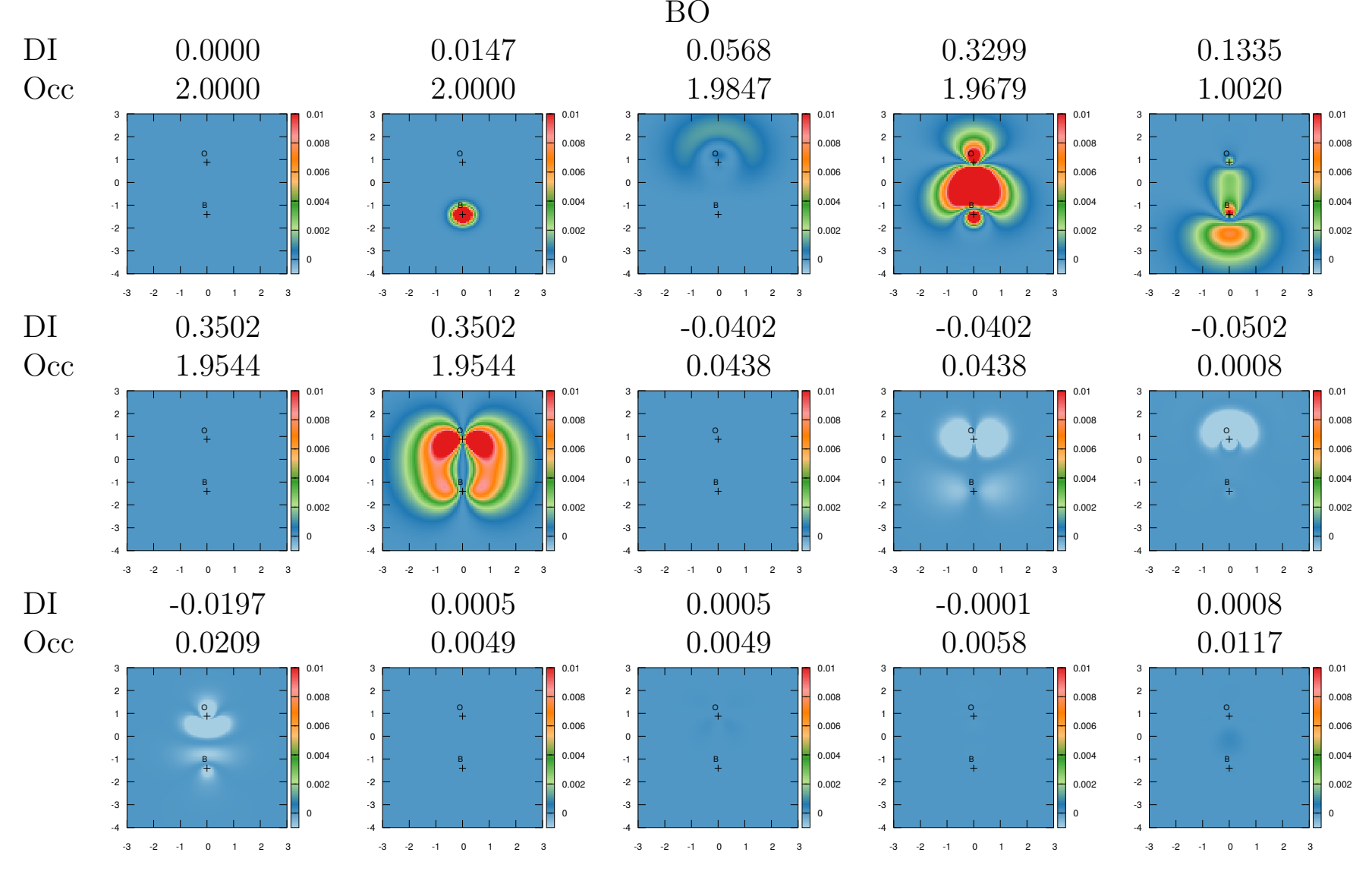


Table S 13: Bonding channels and the corresponding occupation numbers as well as maps of bonding densities for $\mathrm{BO}^{+}$.

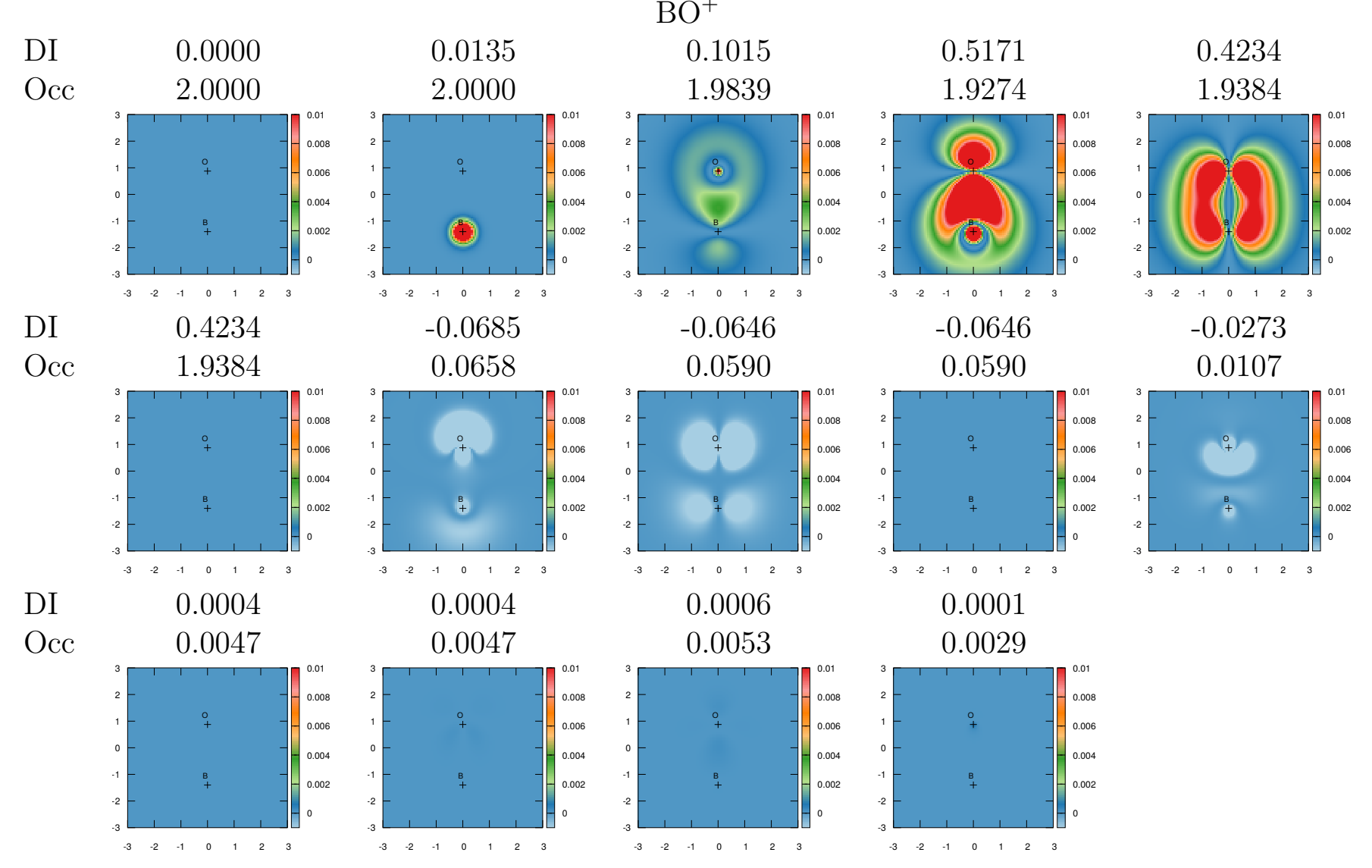


Table S 14: Bonding channels and the corresponding occupation numbers as well as maps of bonding densities for BS.

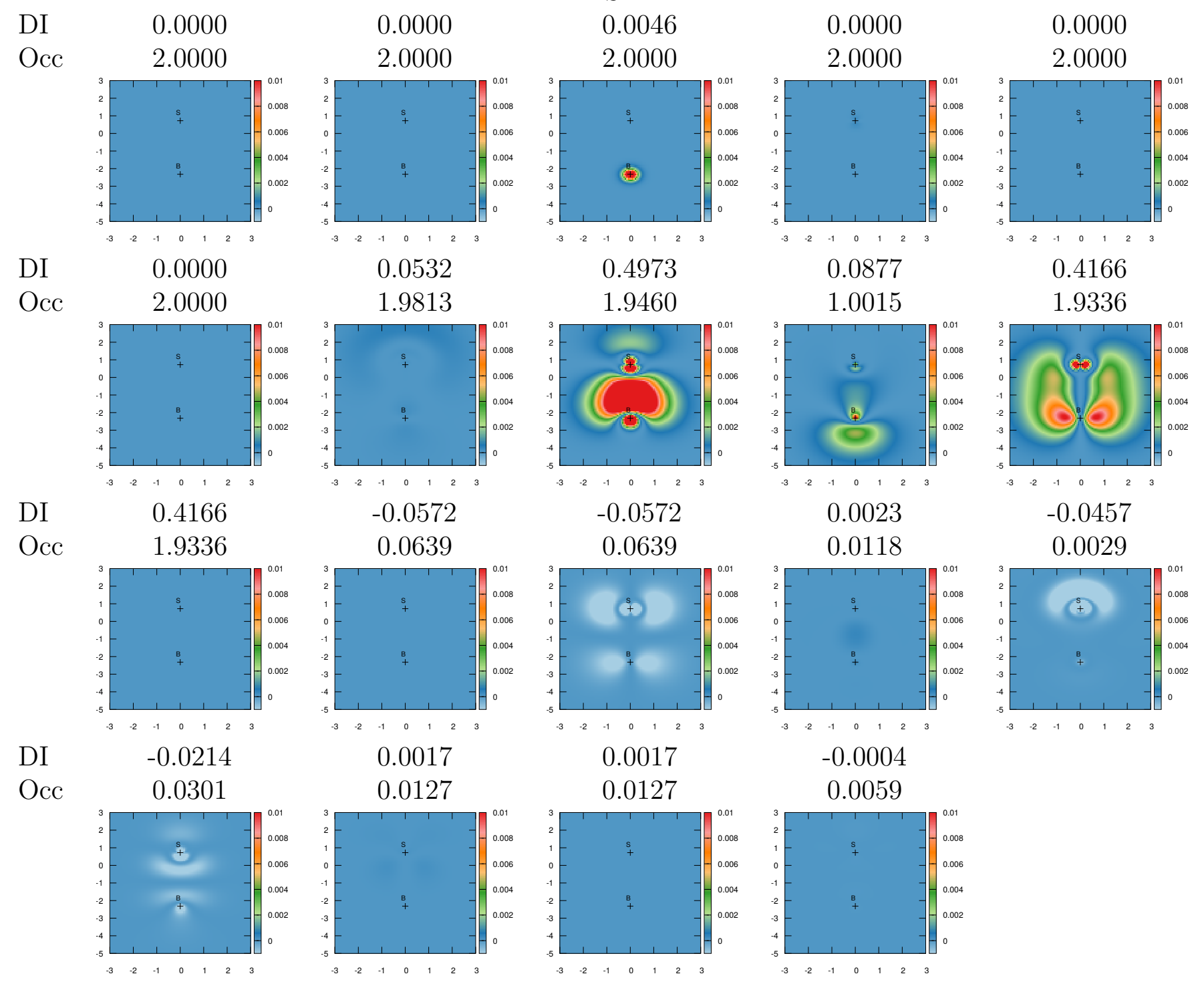


Table S 15: Bonding channels and the corresponding occupation numbers as well as maps of bonding densities for $\mathrm{CaO}$.

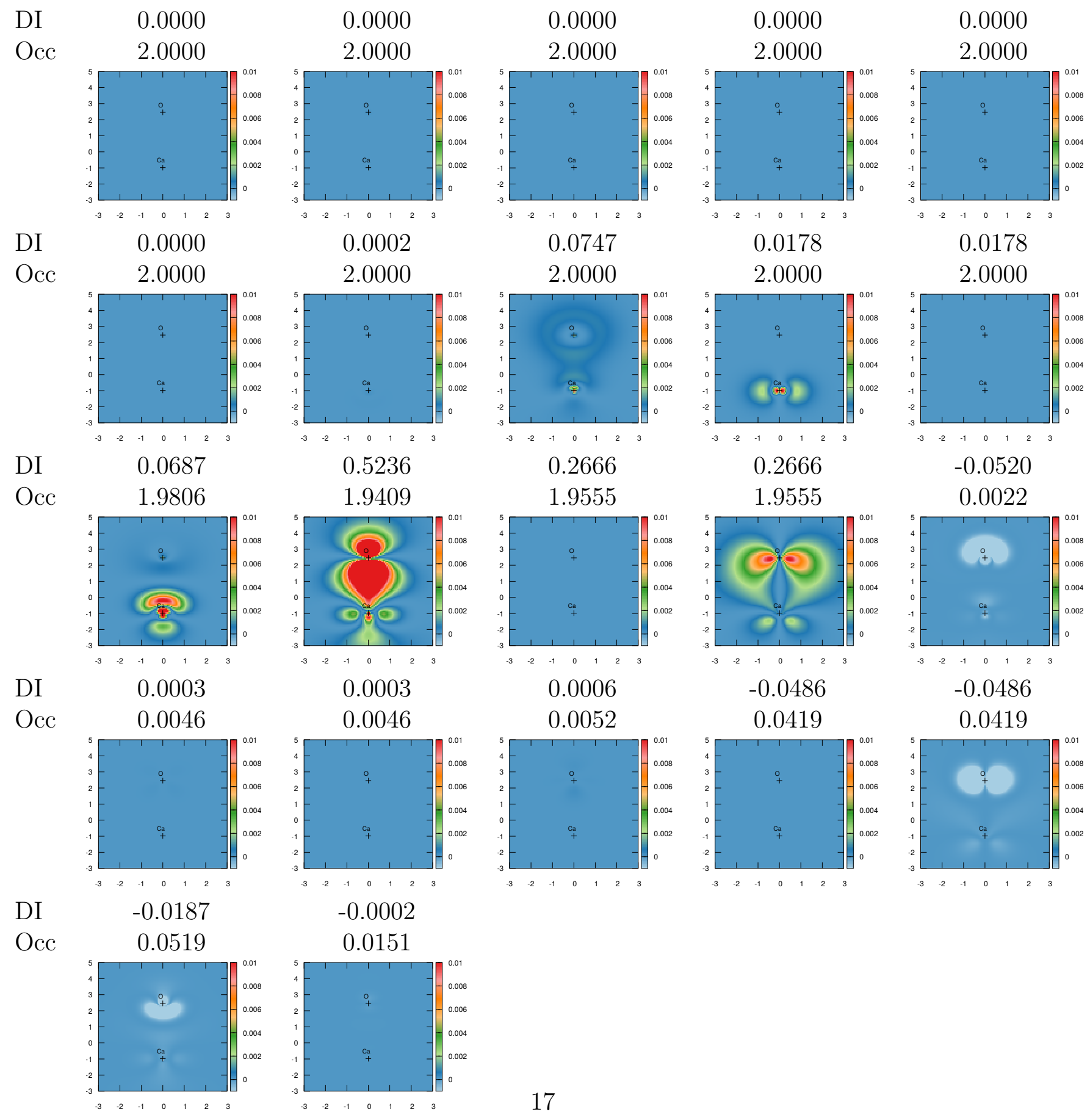


Table S 16: Bonding channels and the corresponding occupation numbers as well as maps of bonding densities for CaS.
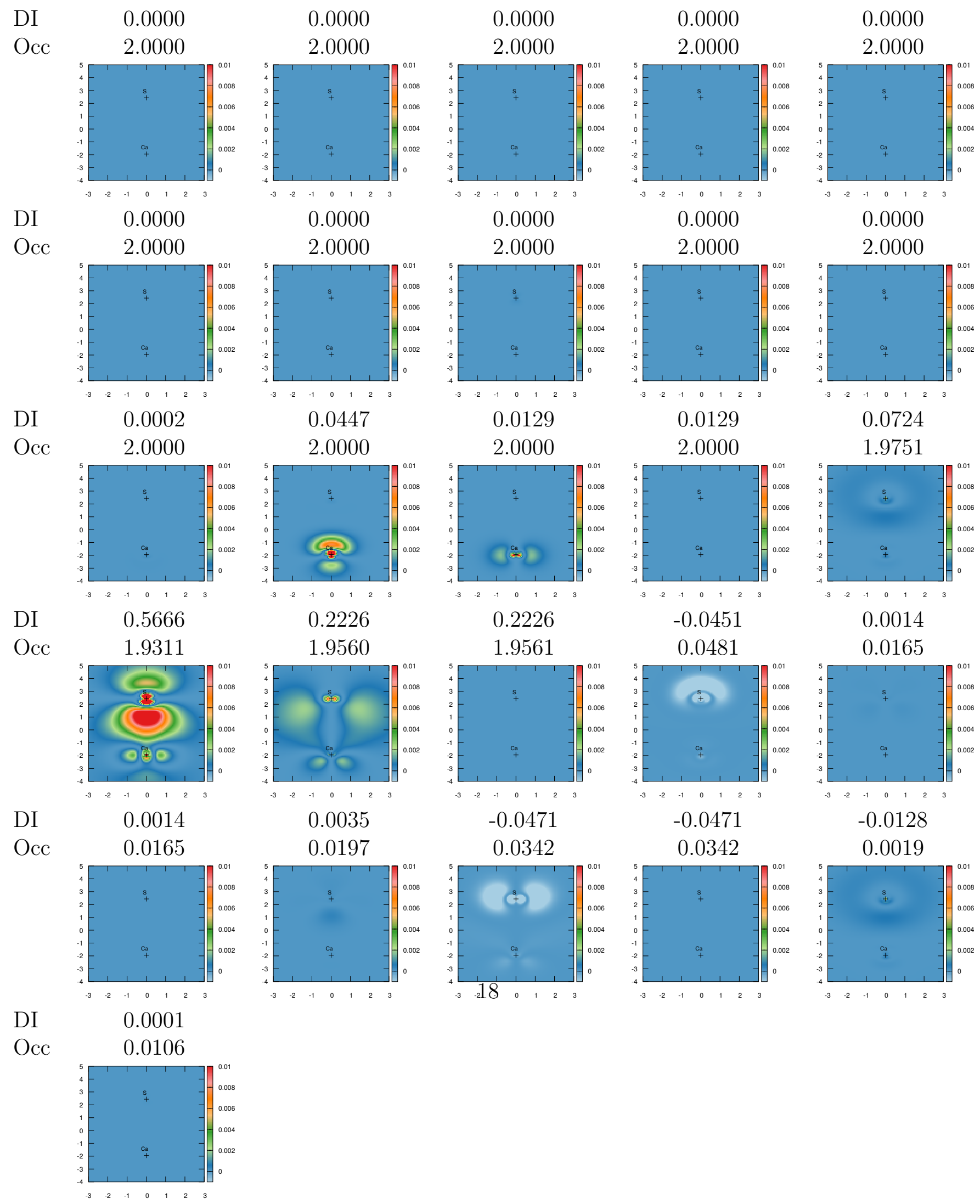
Table S 17: Bonding channels and the corresponding occupation numbers as well as maps of bonding densities for $\mathrm{CN}$.

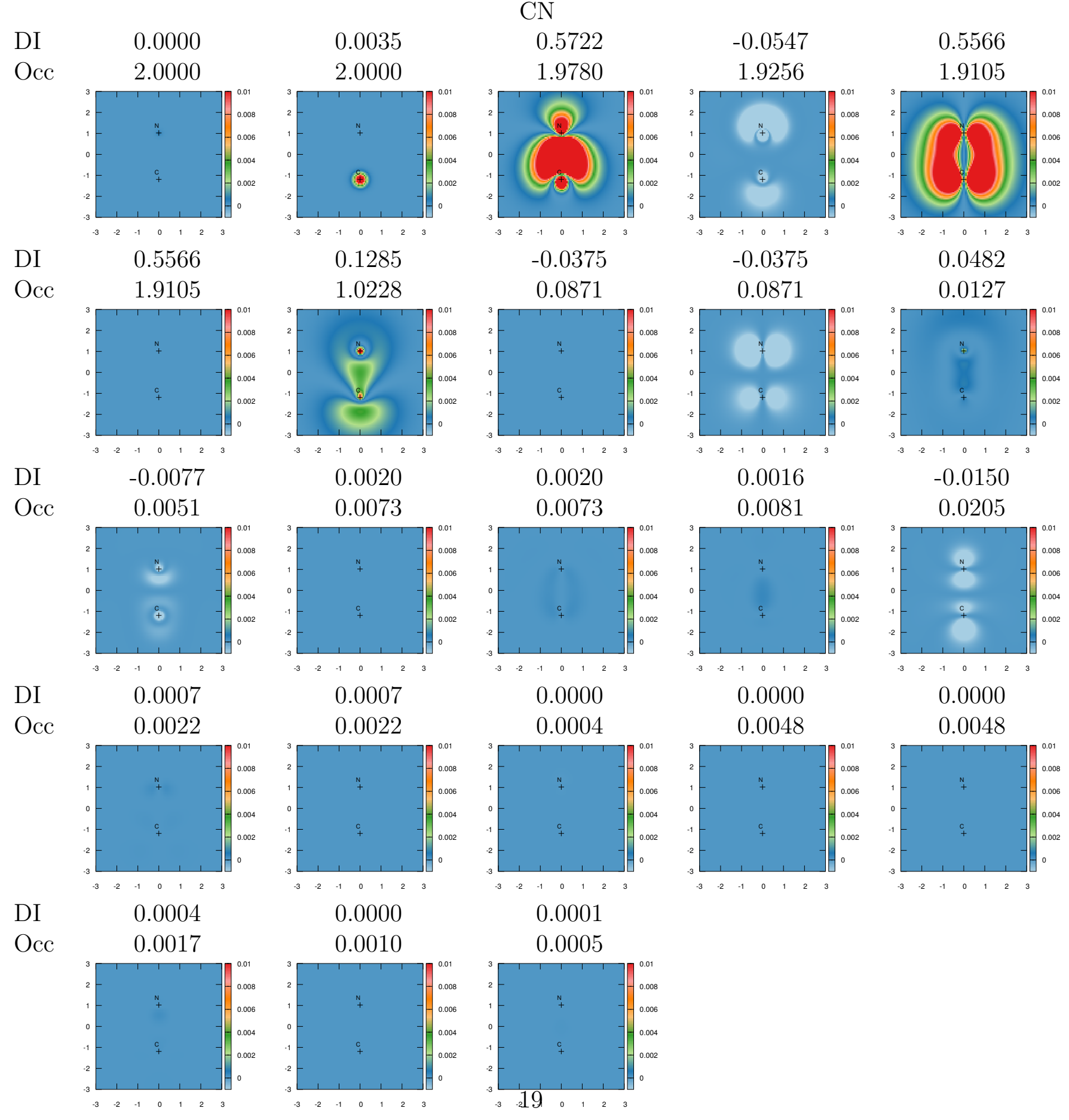


Table S 18: Bonding channels and the corresponding occupation numbers as well as maps of bonding densities for $\mathrm{CN}^{+}$.

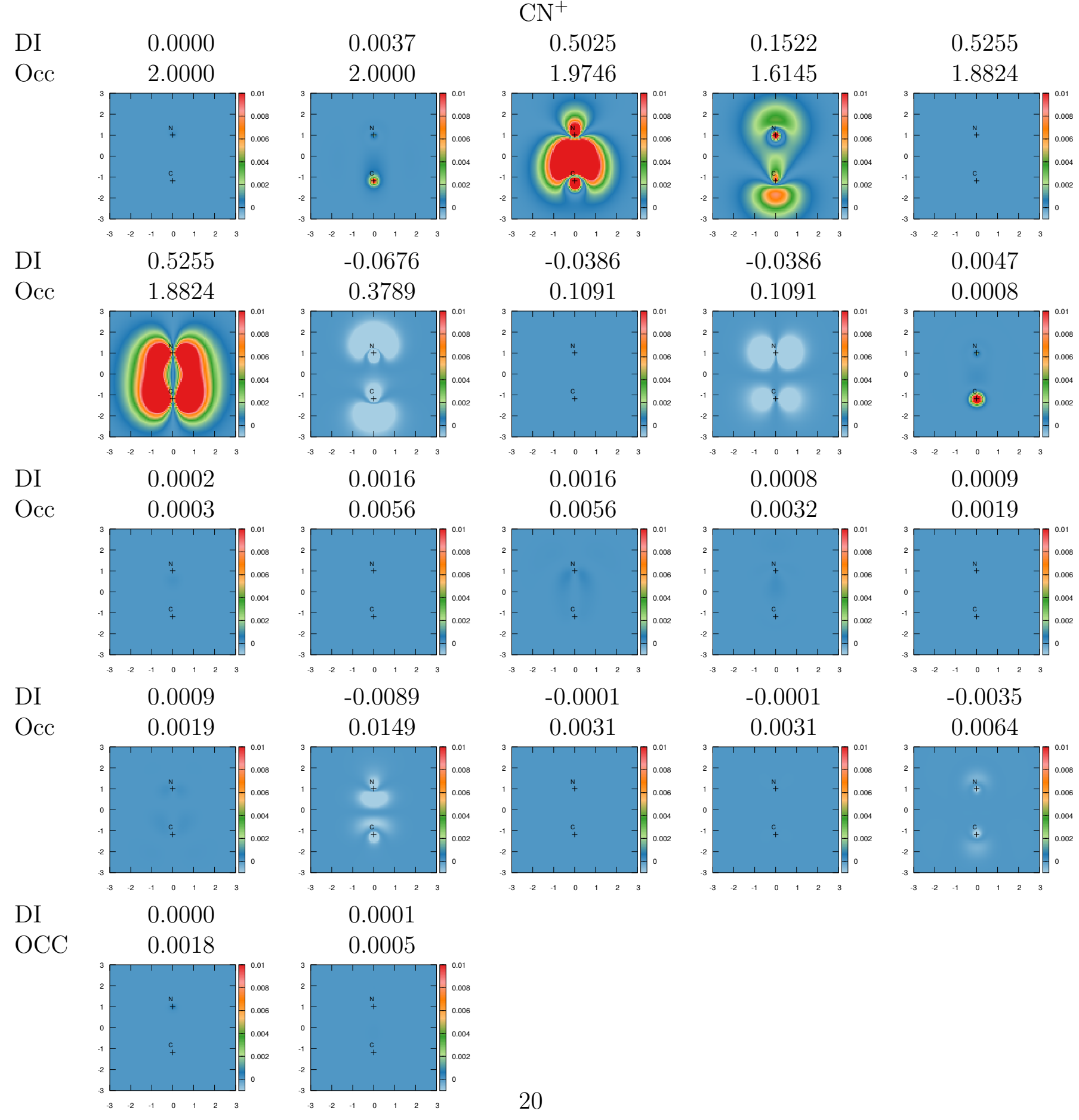


Table S 19: Bonding channels and the corresponding occupation numbers as well as maps of bonding densities for $\mathrm{CO}$.

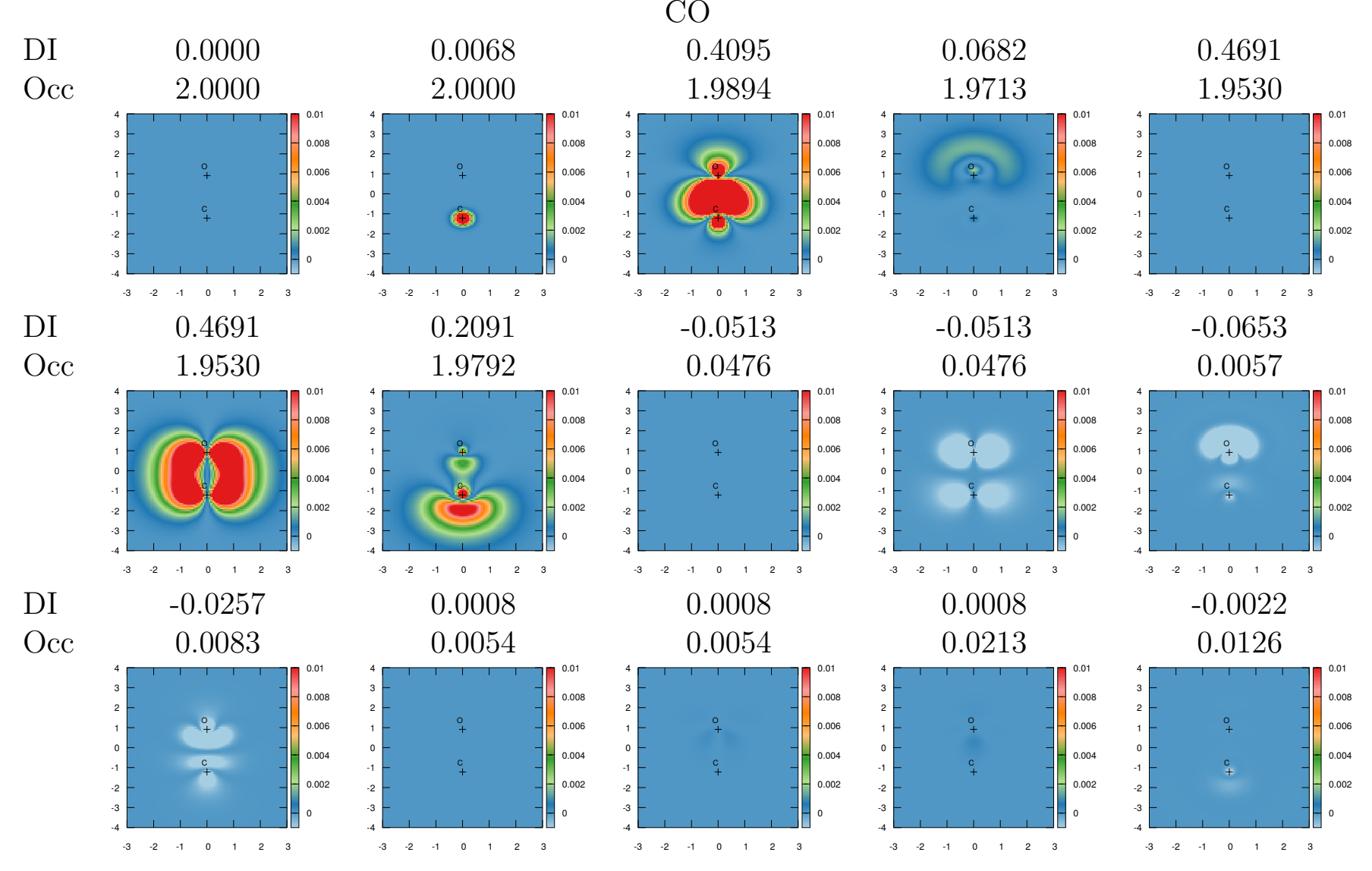


Table S 20: Bonding channels and the corresponding occupation numbers as well as maps of bonding densities for $\mathrm{CO}^{+}$.

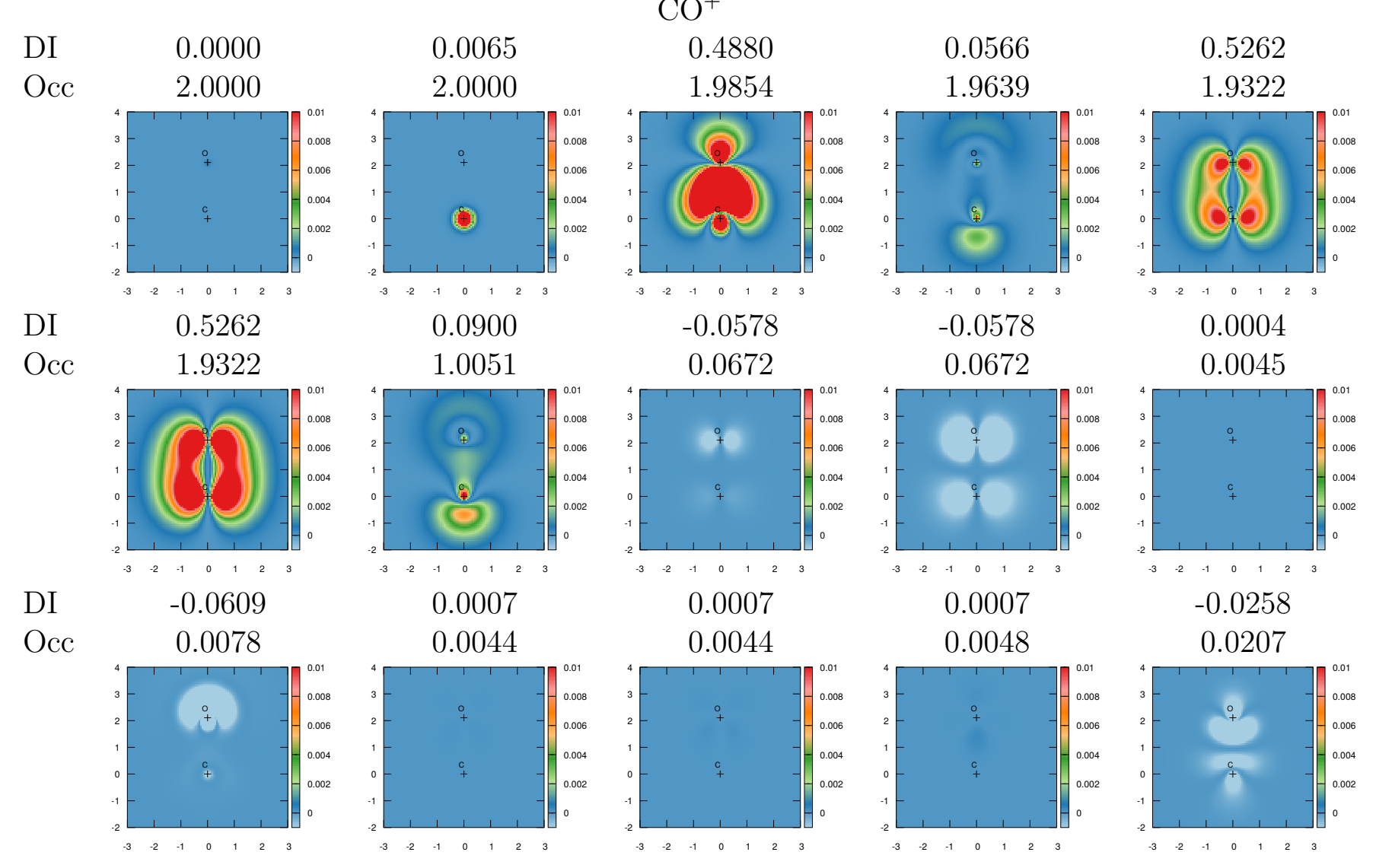


Table S 21: Bonding channels and the corresponding occupation numbers as well as maps of bonding densities for CSe.

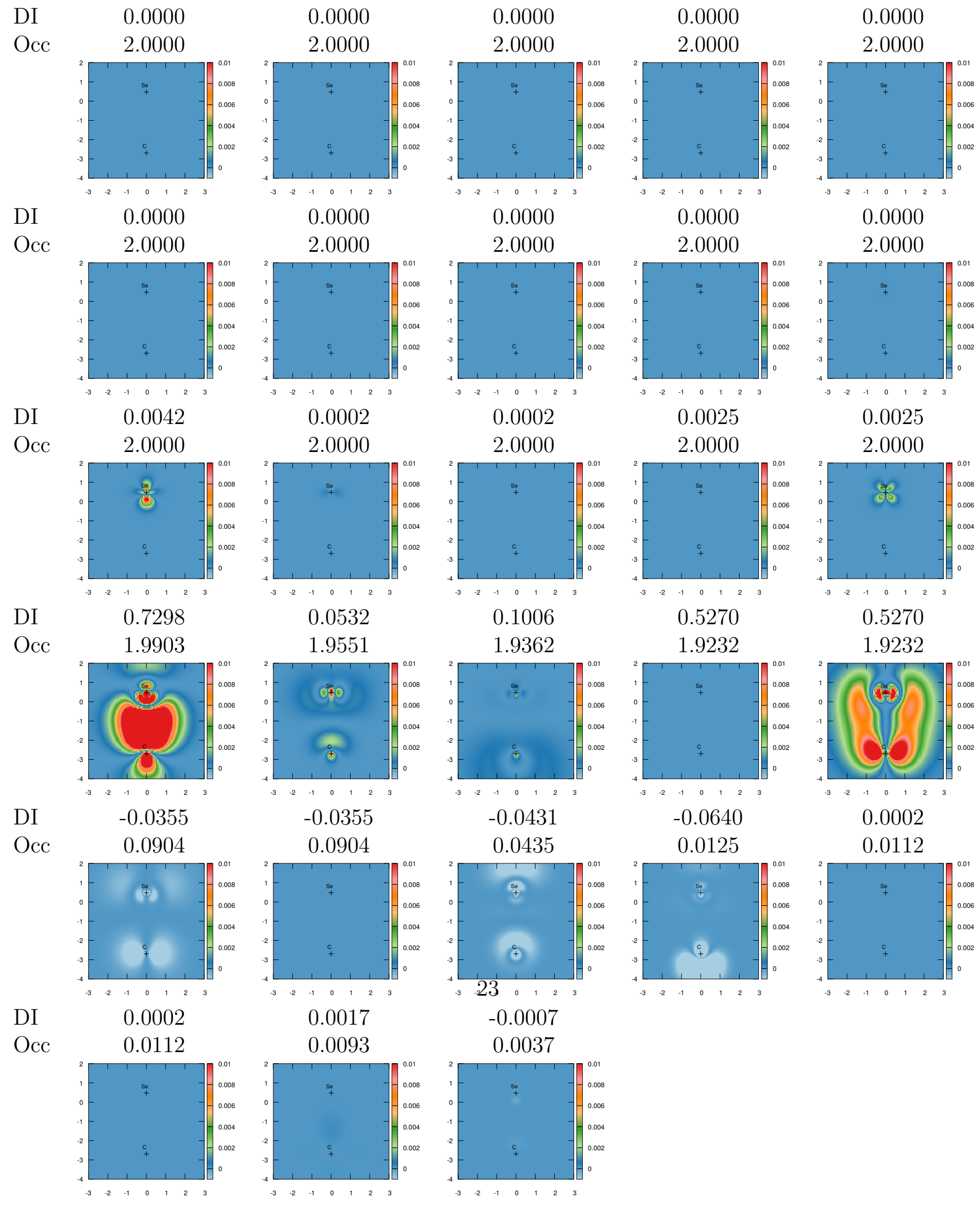


Table S 22: Bonding channels and the corresponding occupation numbers as well as maps of bonding densities for CS.

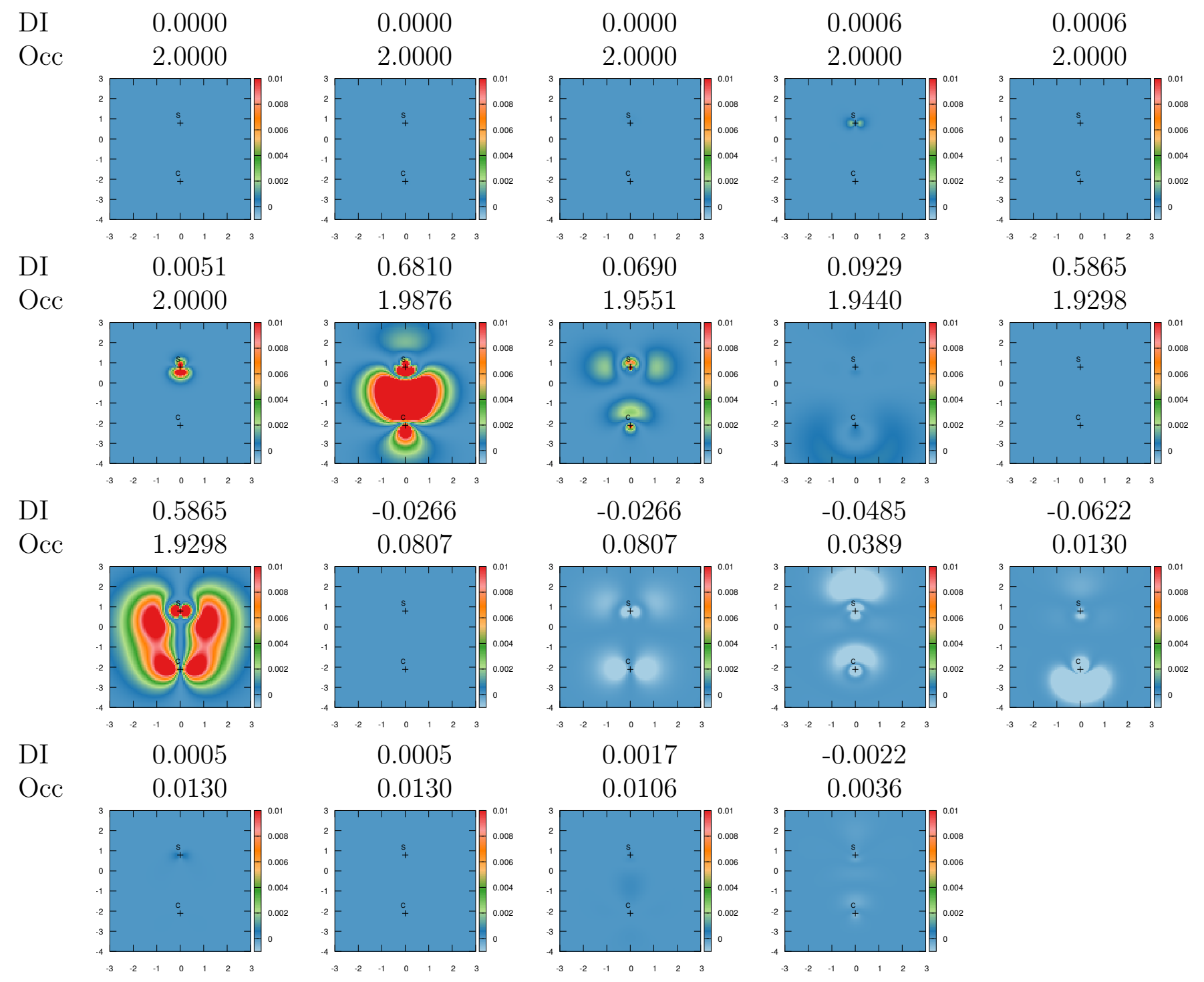


Table S 23: Bonding channels and the corresponding occupation numbers as well as maps of bonding densities for $\mathrm{GaO}$.

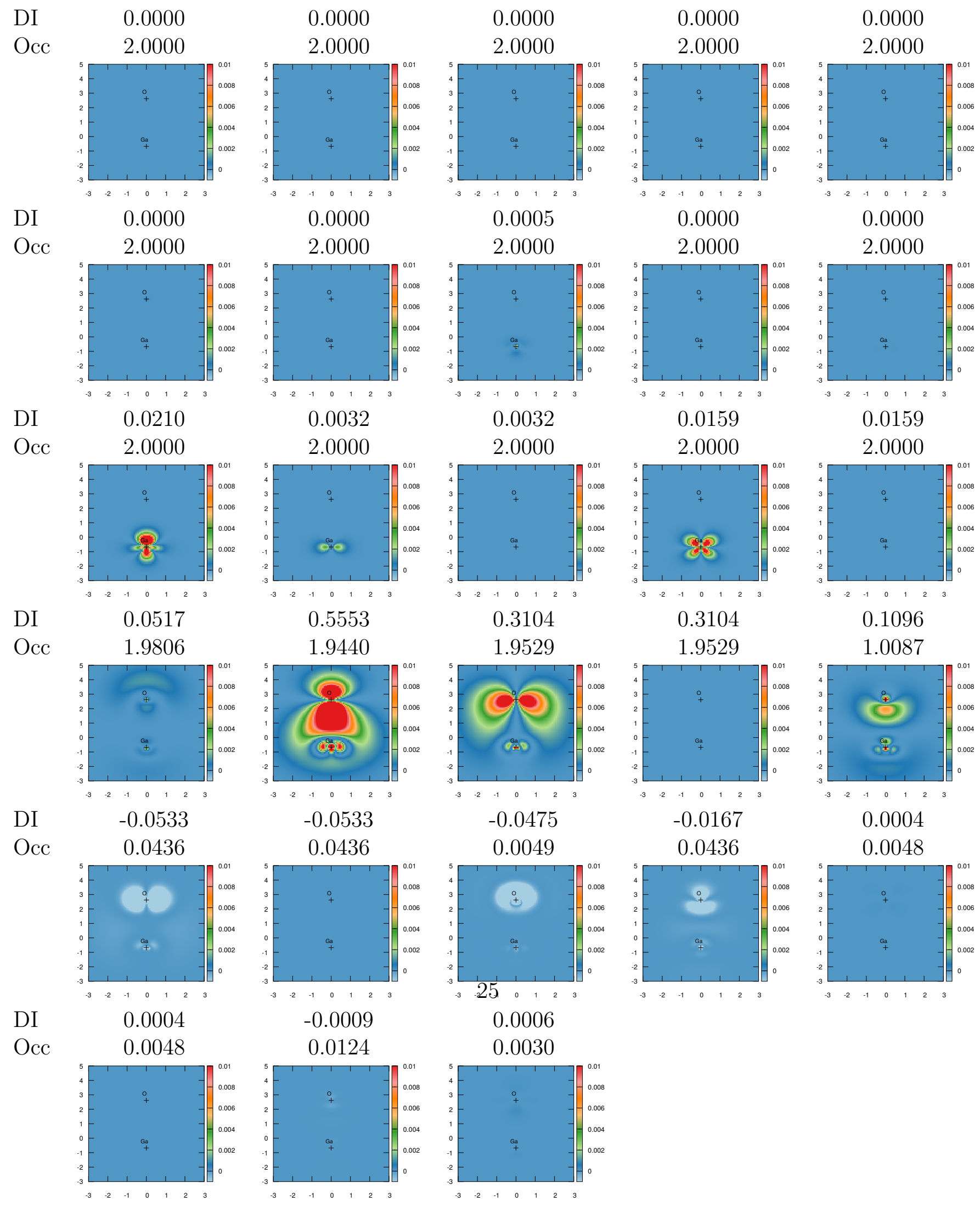


Table S 24: Bonding channels and the corresponding occupation numbers as well as maps of bonding densities for $\mathrm{GeO}$.

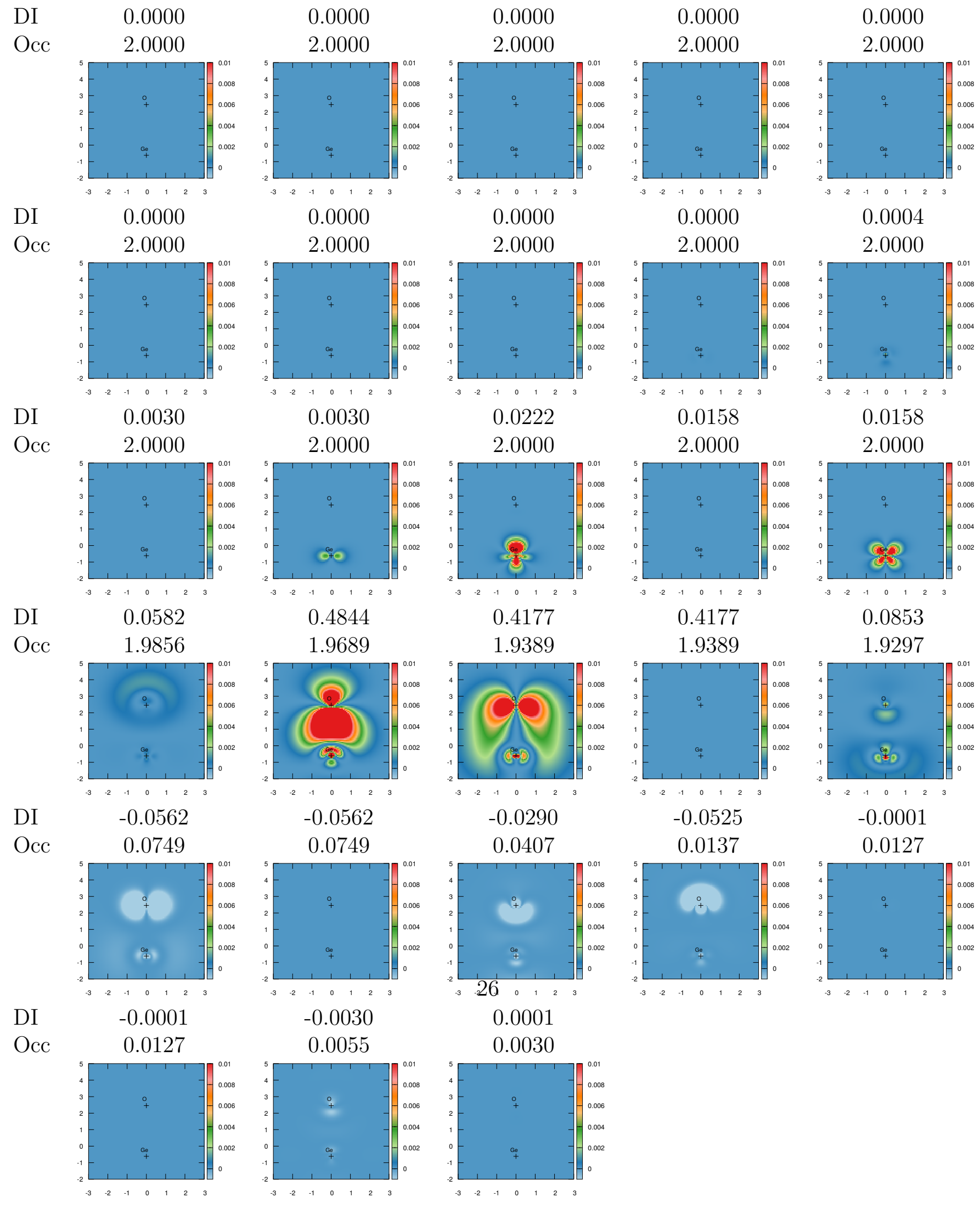


Table S 25: Bonding channels and the corresponding occupation numbers as well as maps of bonding densities for GeSe, part one.

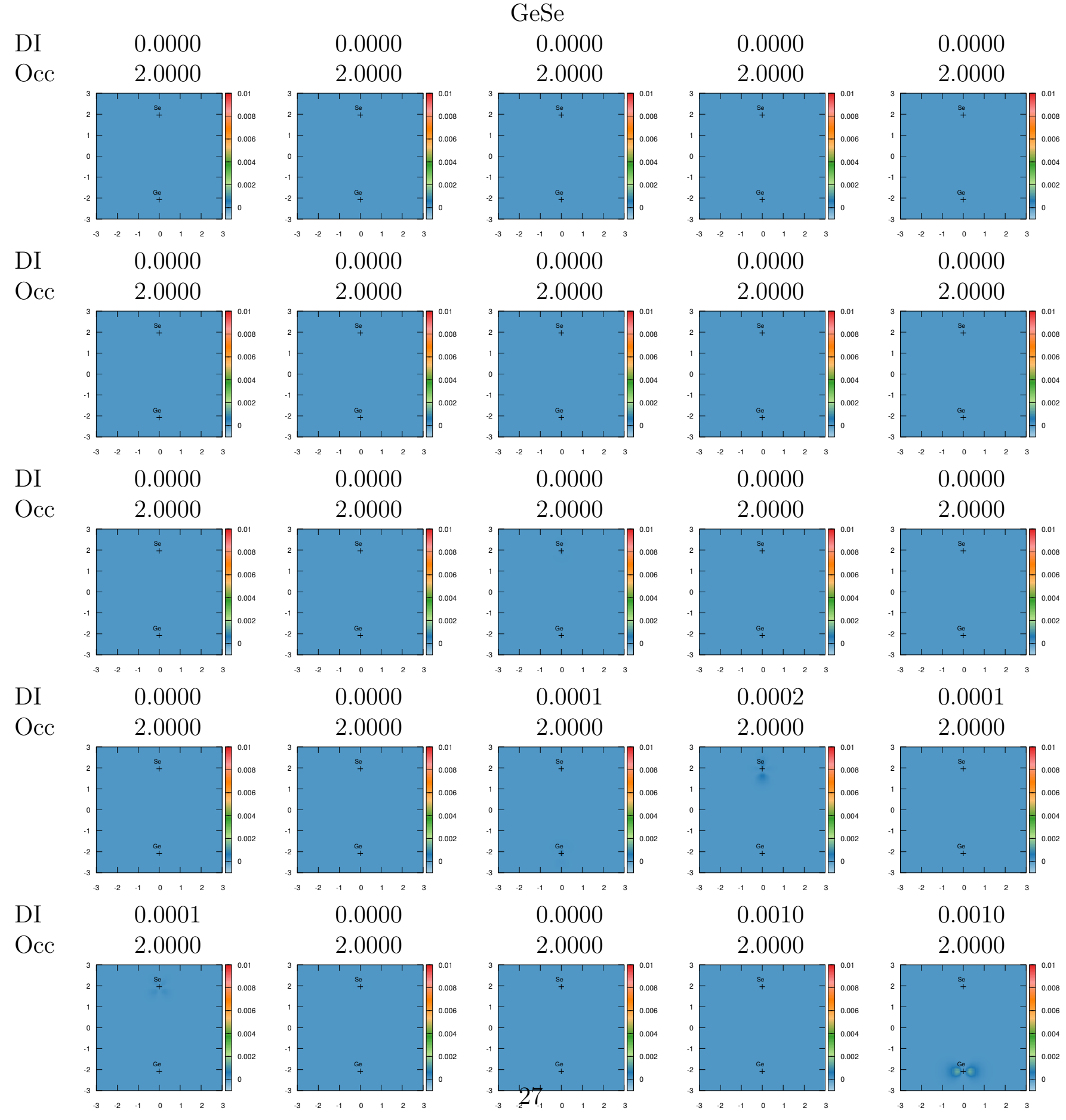


Table S 26: Bonding channels and the corresponding occupation numbers as well as maps of bonding densities for GeSe, part two.

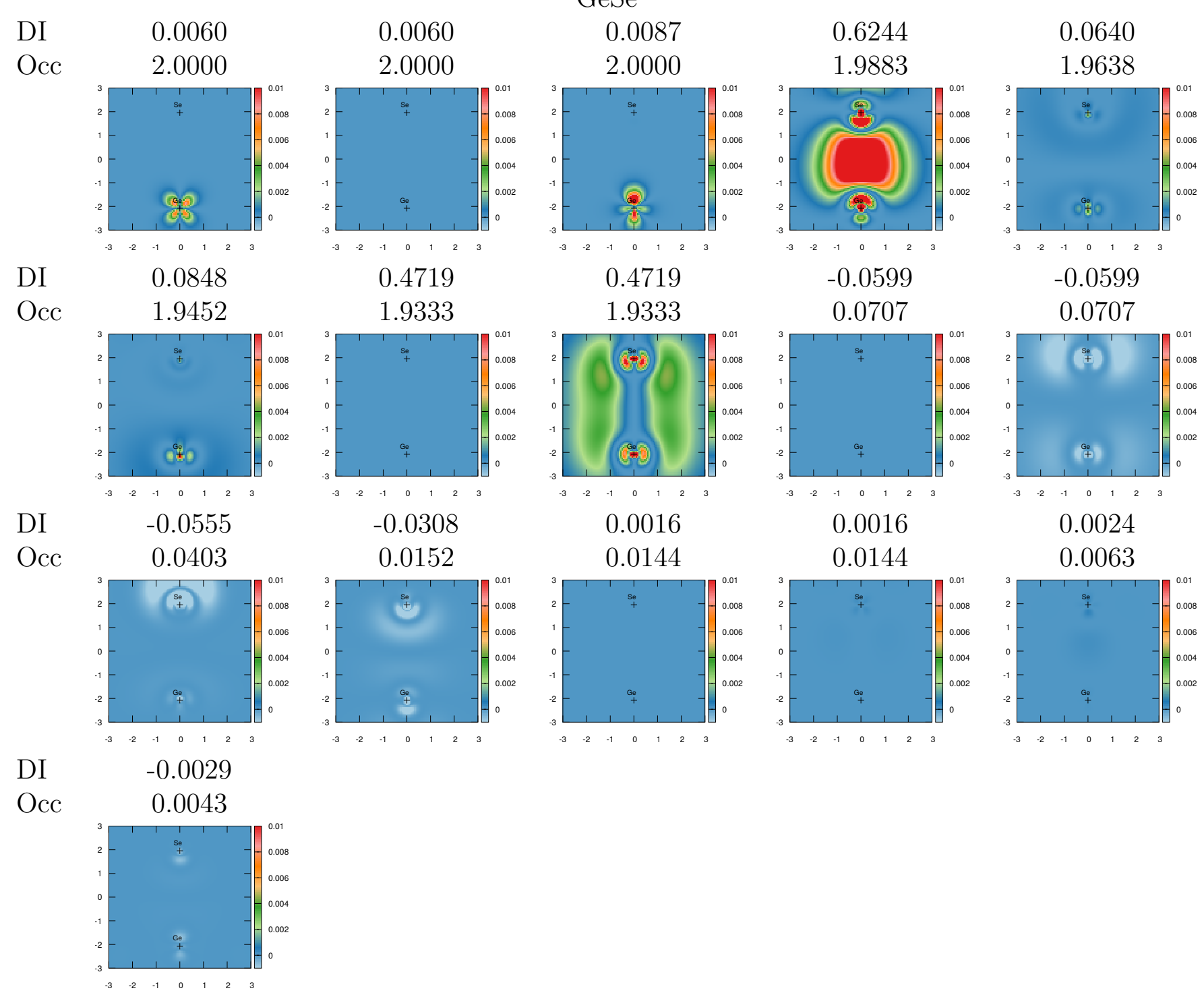


Table S 27: Bonding channels and the corresponding occupation numbers as well as maps of bonding densities for $\mathrm{MgO}$.

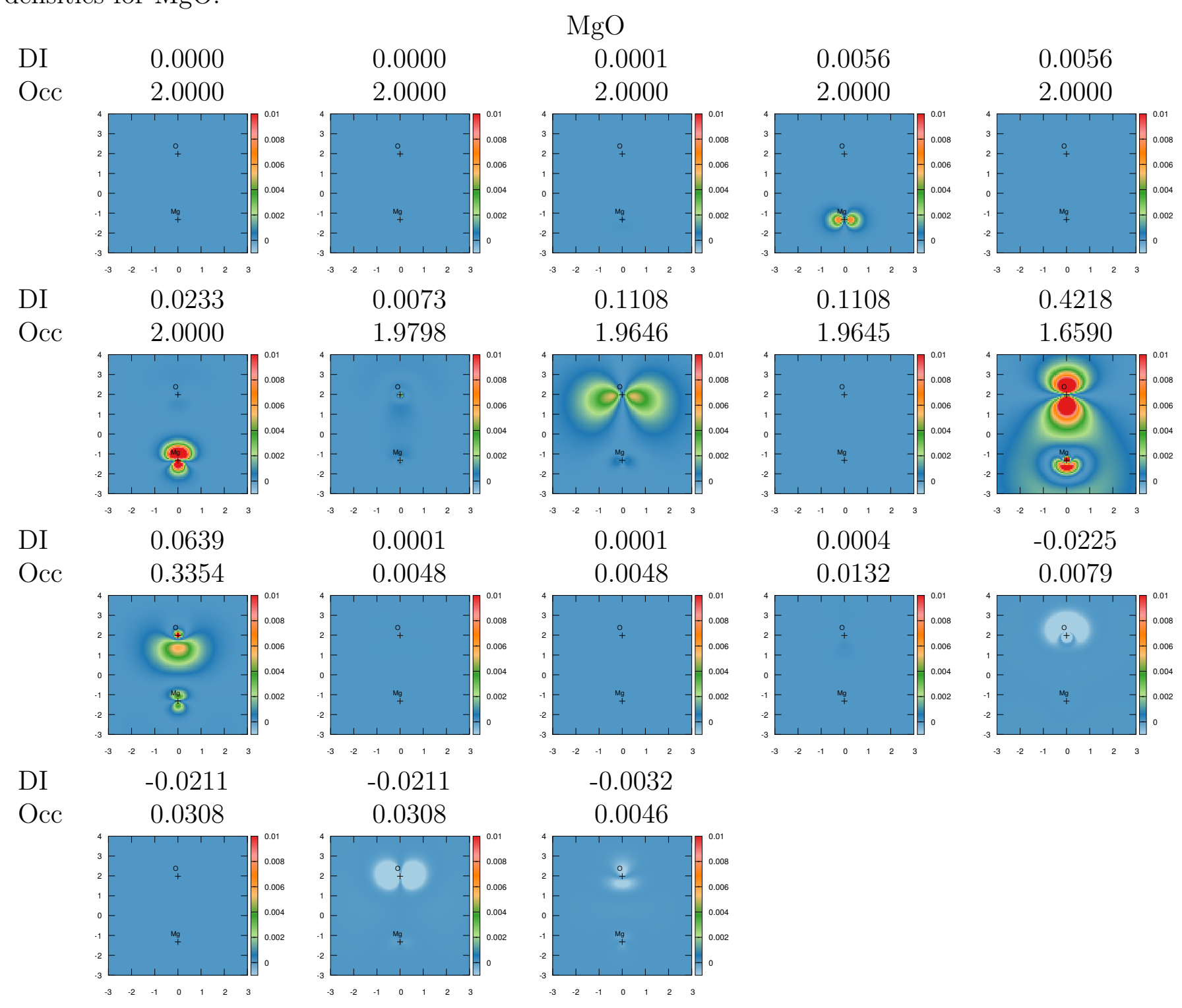


Table S 28: Bonding channels and the corresponding occupation numbers as well as maps of bonding densities for MgS.

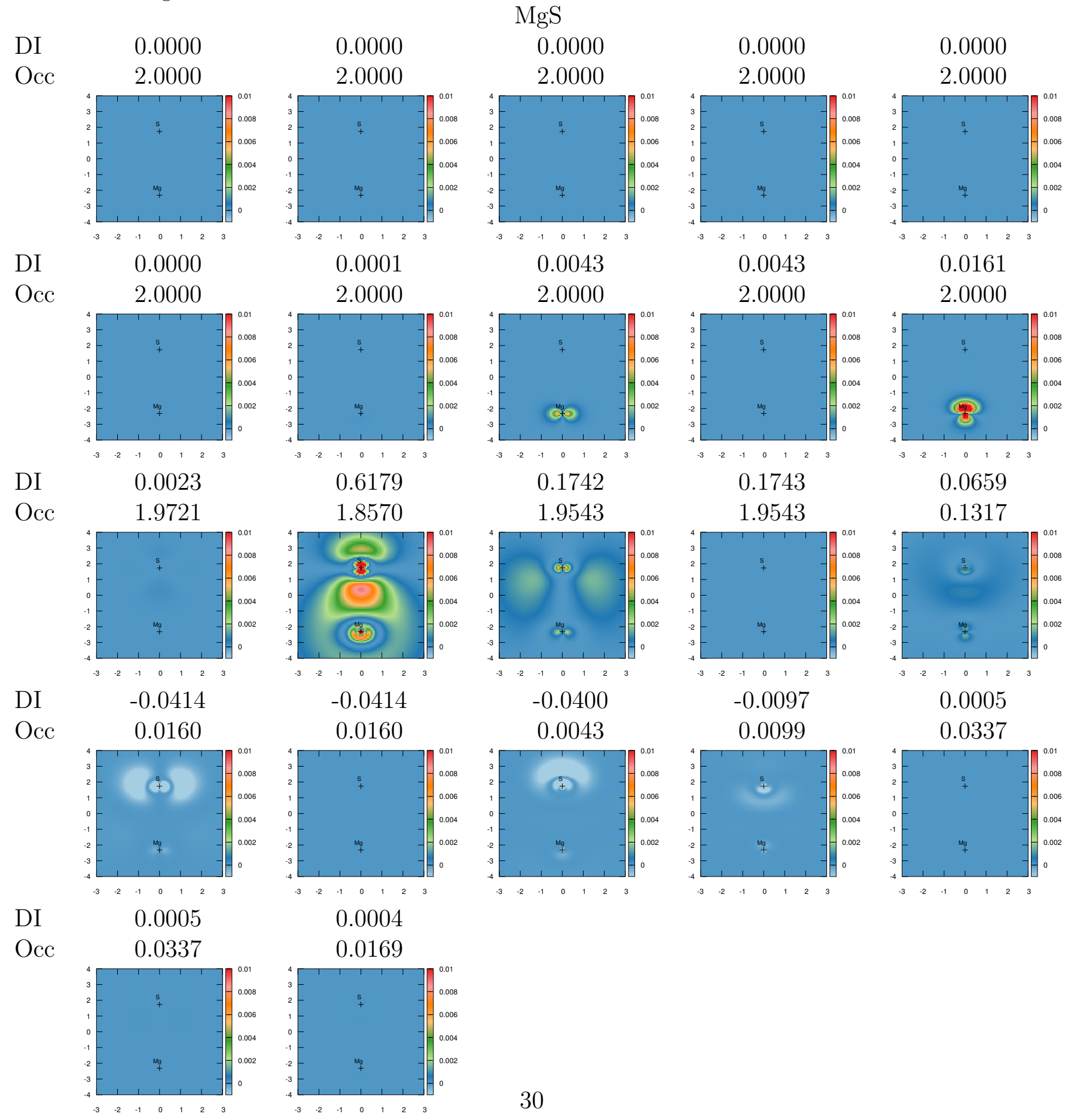


Table S 29: Bonding channels and the corresponding occupation numbers as well as maps of bonding densities for $\mathrm{NO}^{-}$.

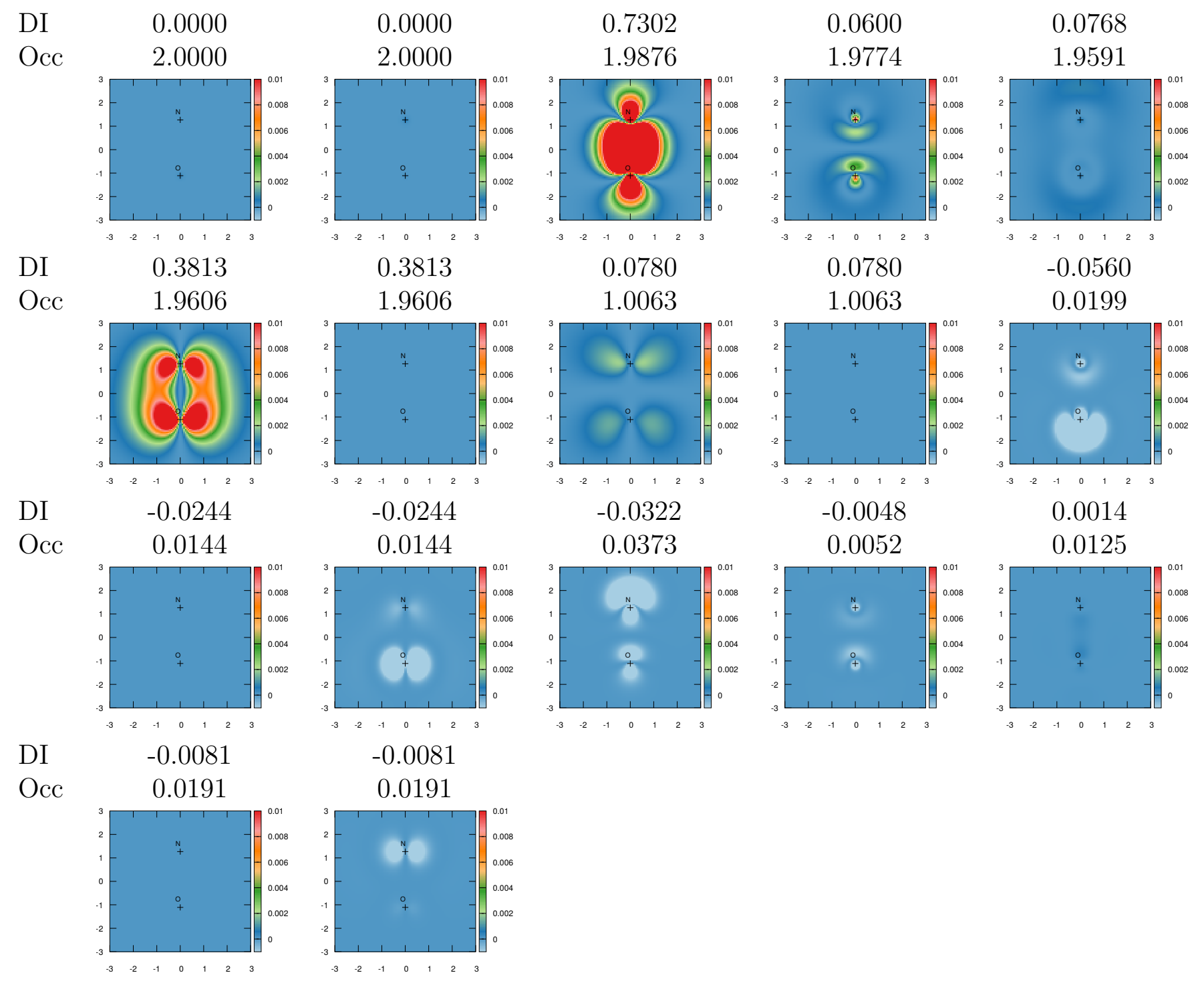


Table S 30: Bonding channels and the corresponding occupation numbers as well as maps of bonding densities for $\mathrm{NO}^{+}$.

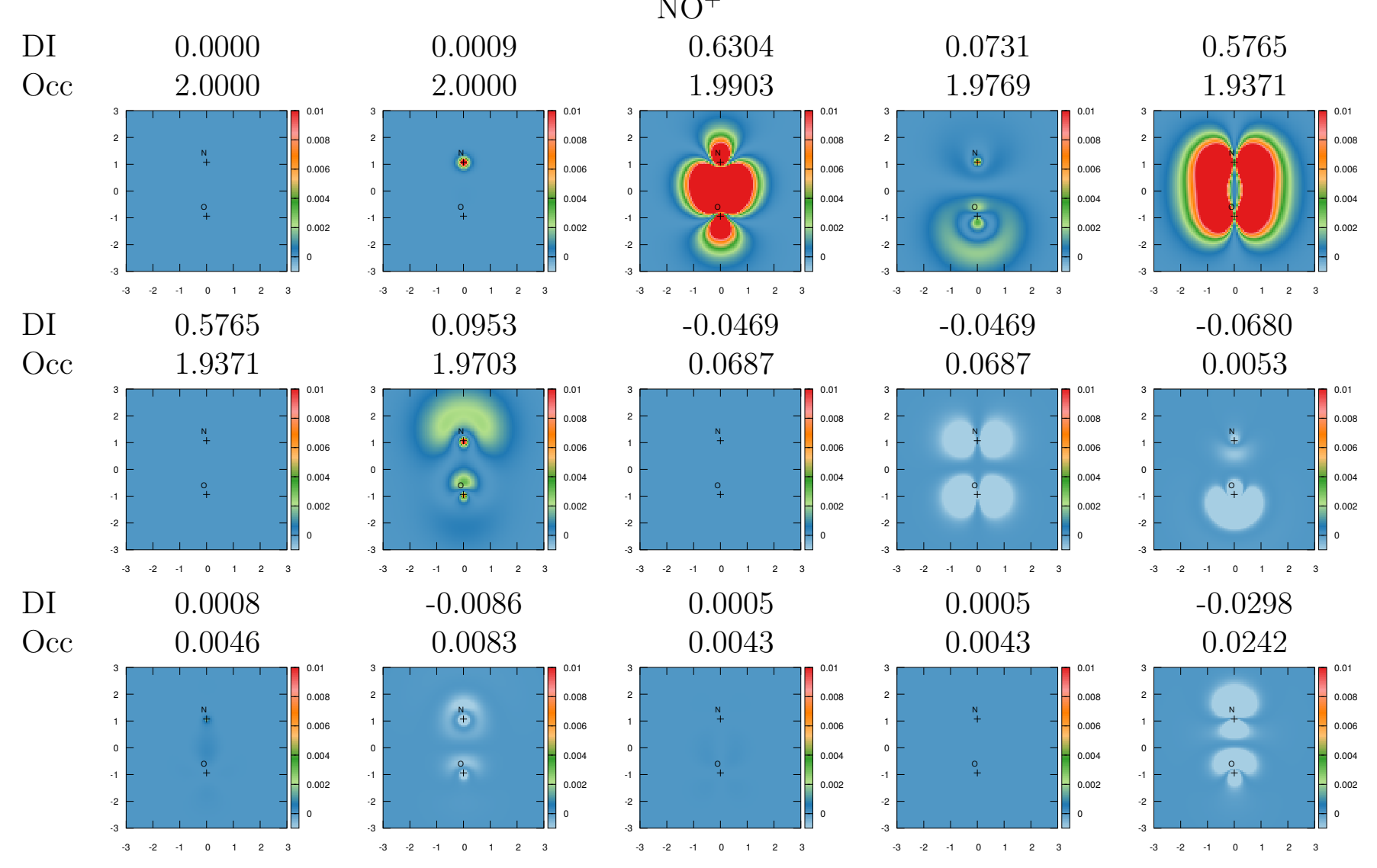


Table S 31: Bonding channels and the corresponding occupation numbers as well as maps of bonding densities for SeO.

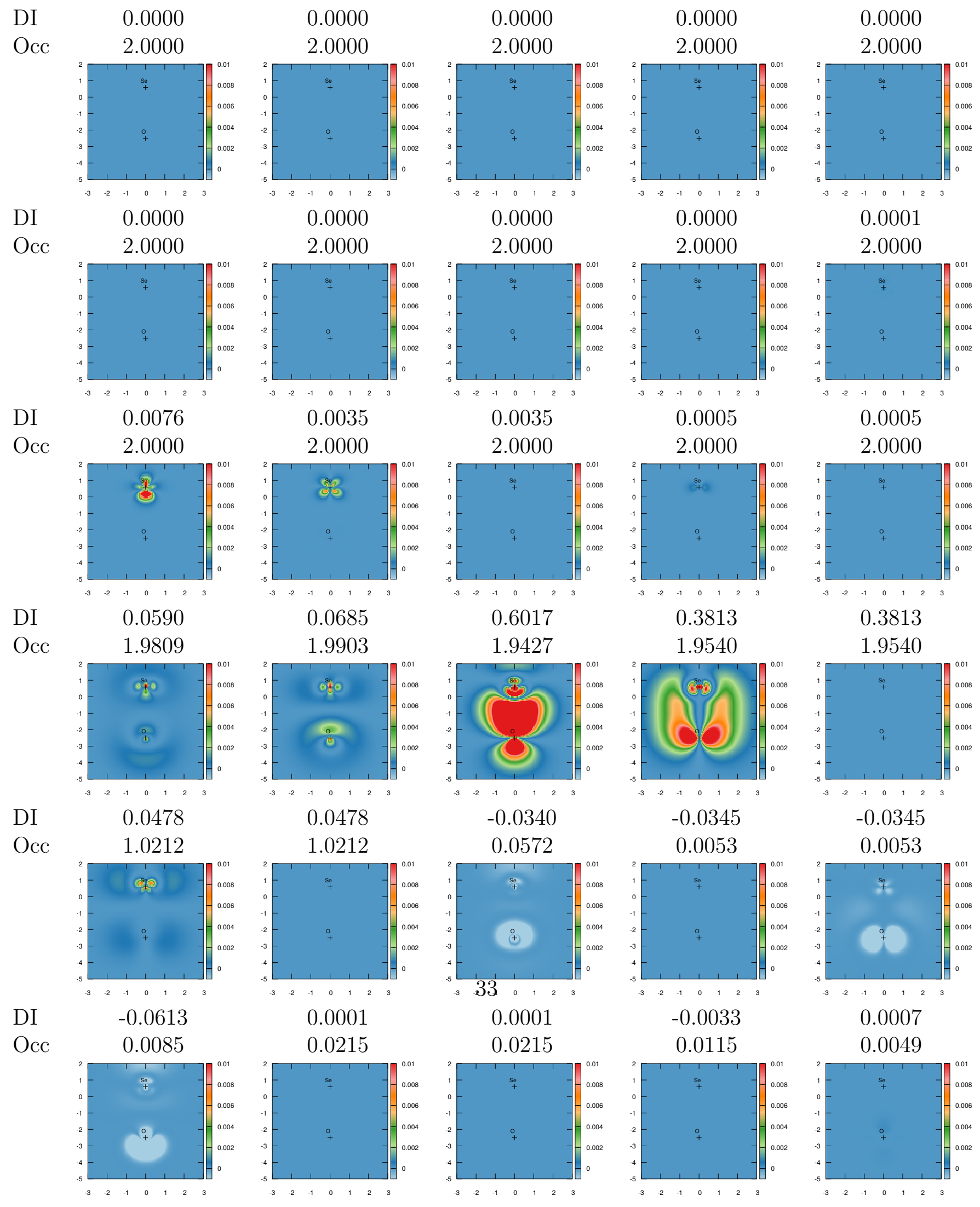


Table S 32: Bonding channels and the corresponding occupation numbers as well as maps of bonding densities for SeS, part one.

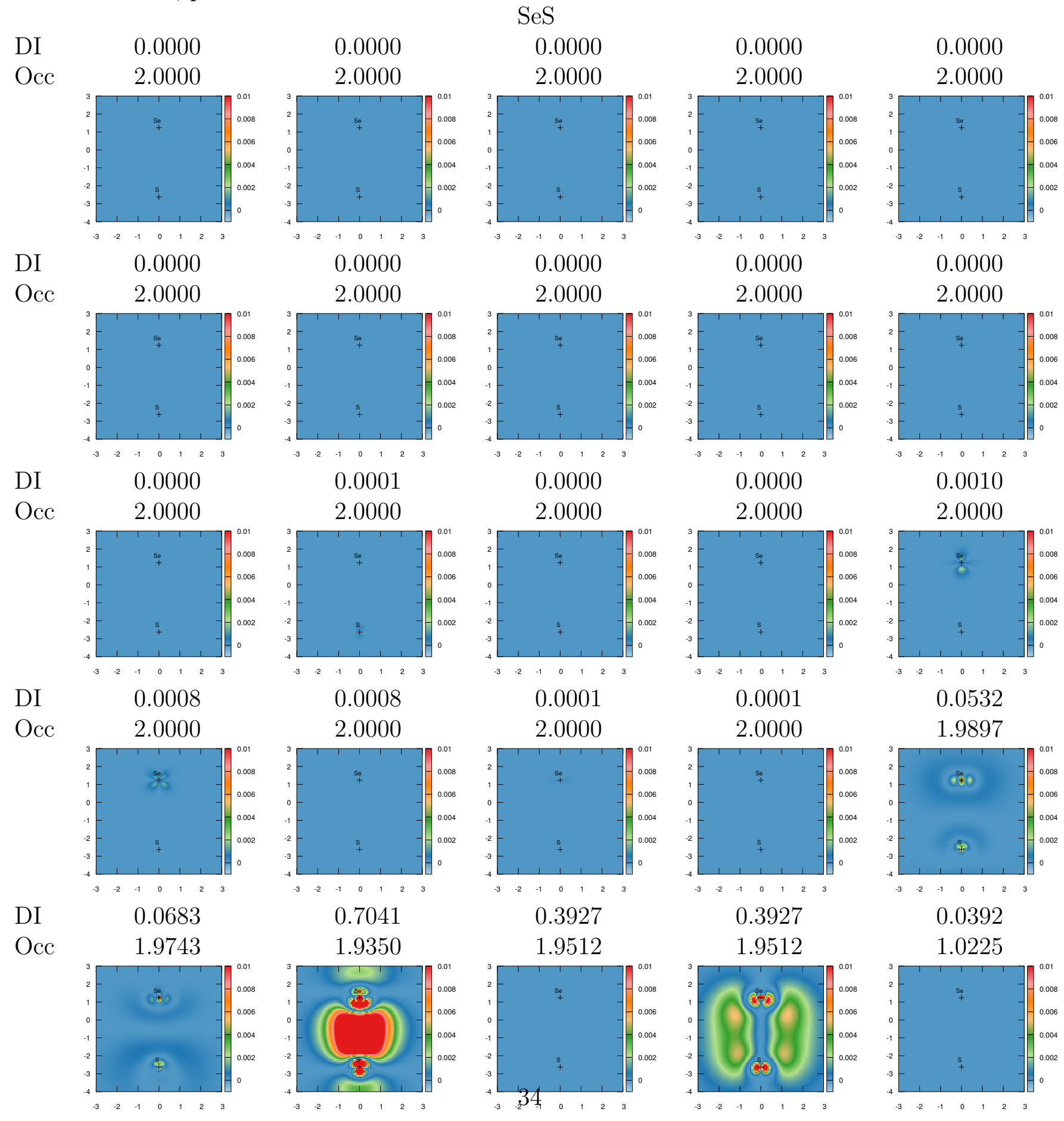


Table S 33: Bonding channels and the corresponding occupation numbers as well as maps of bonding densities for SeS, part two.

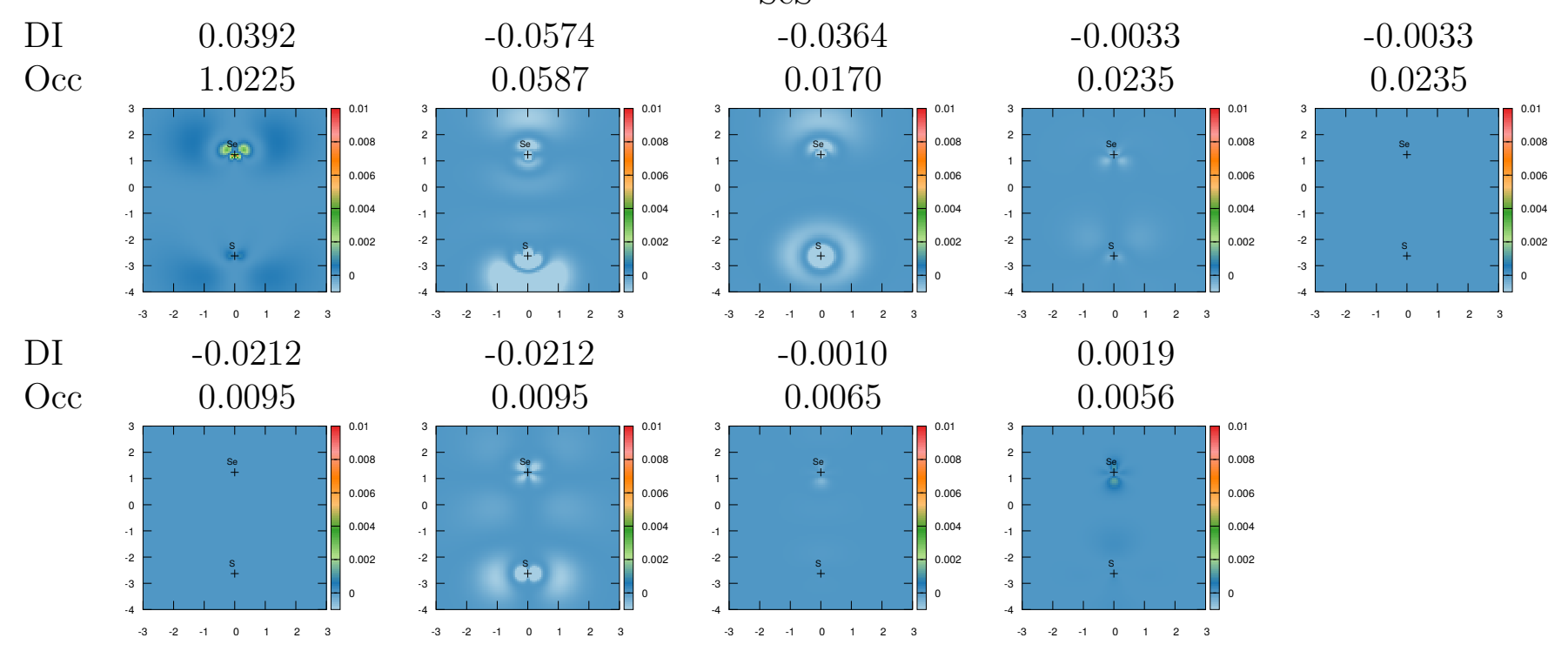


Table S 34: Bonding channels and the corresponding occupation numbers as well as maps of bonding densities for $\mathrm{SiO}$.

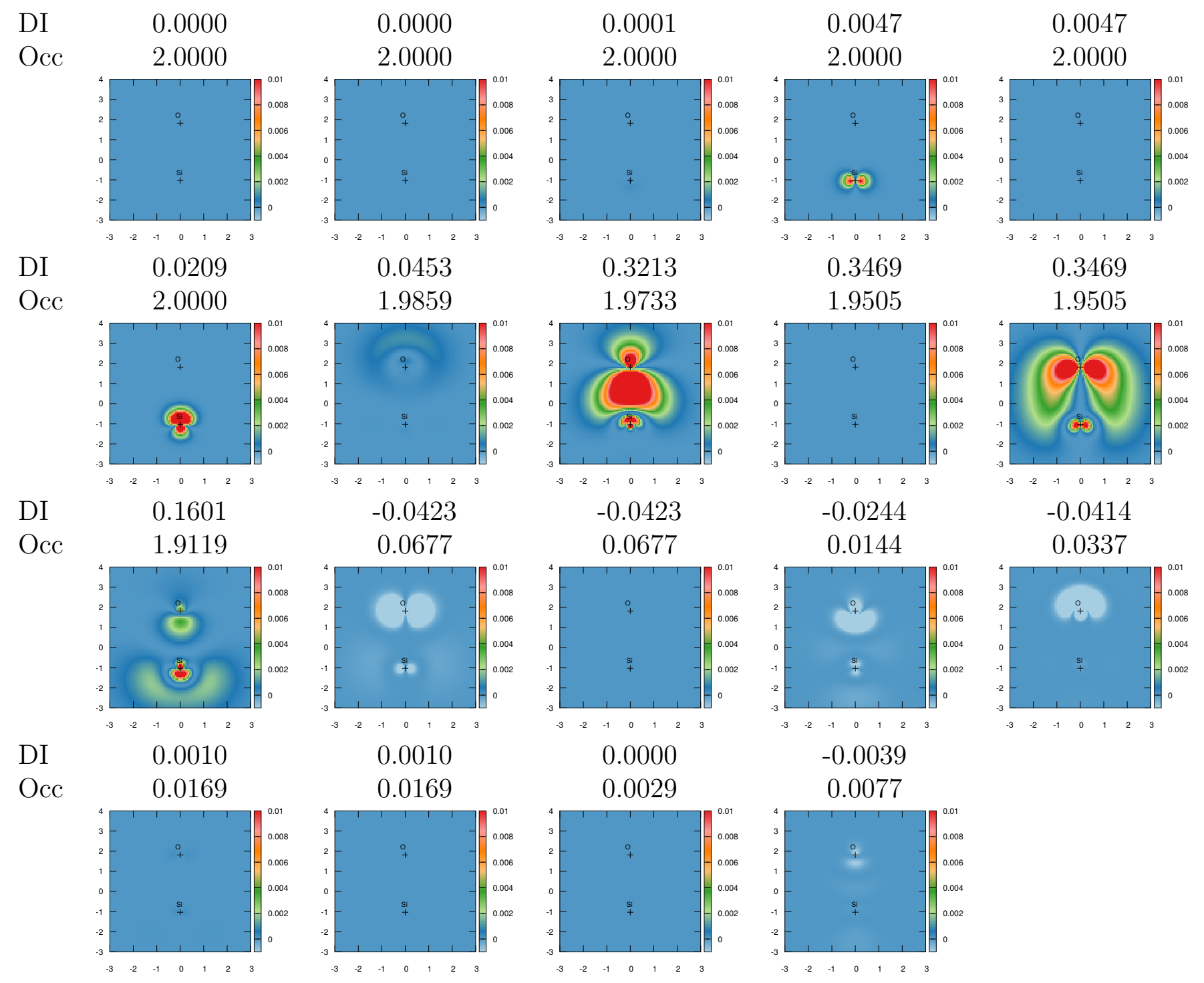


Table S 35: Bonding channels and the corresponding occupation numbers as well as maps of bonding densities for SiS.

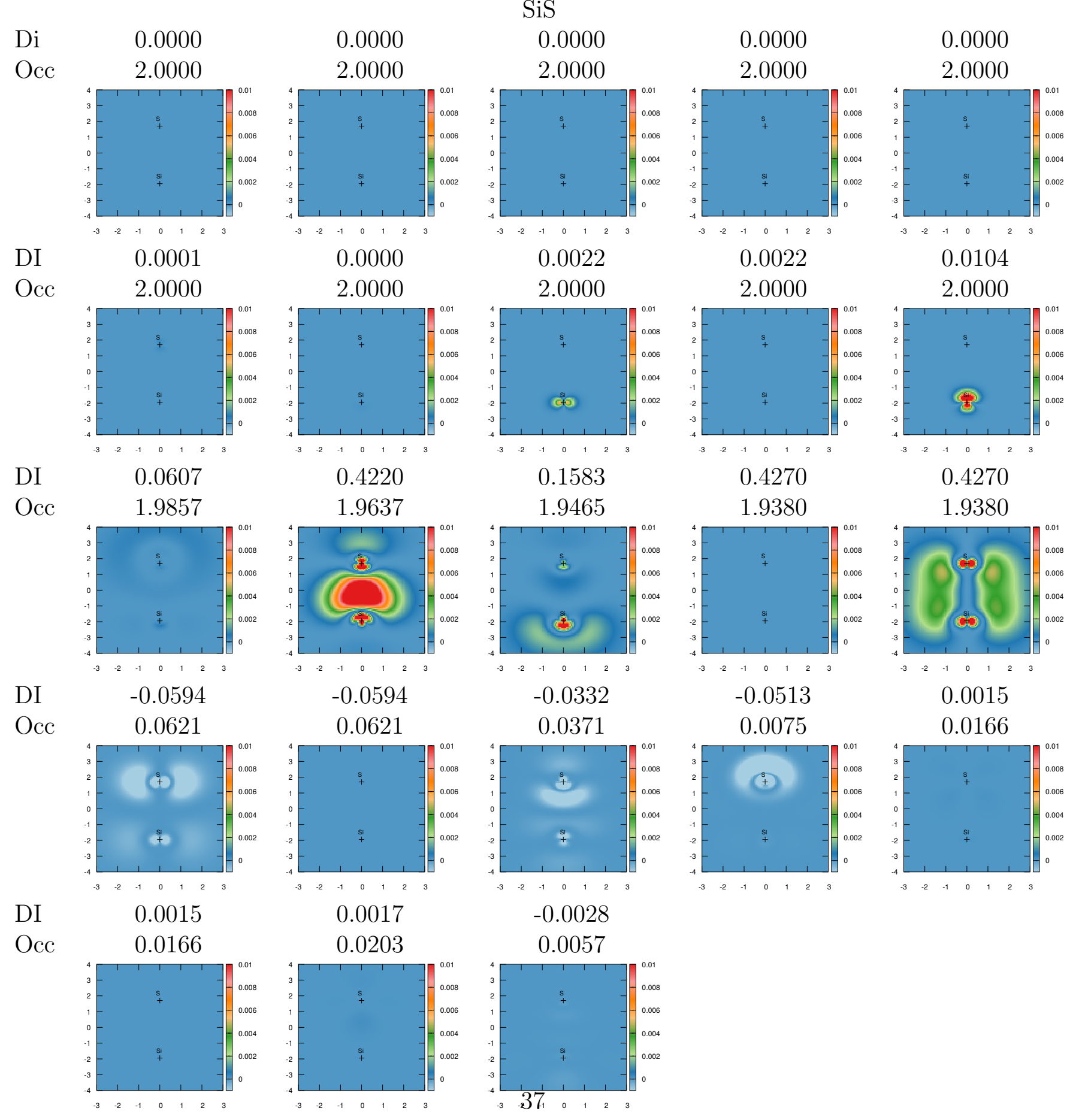


Table S 36: Bonding channels and the corresponding occupation numbers as well as maps of bonding densities for SO.

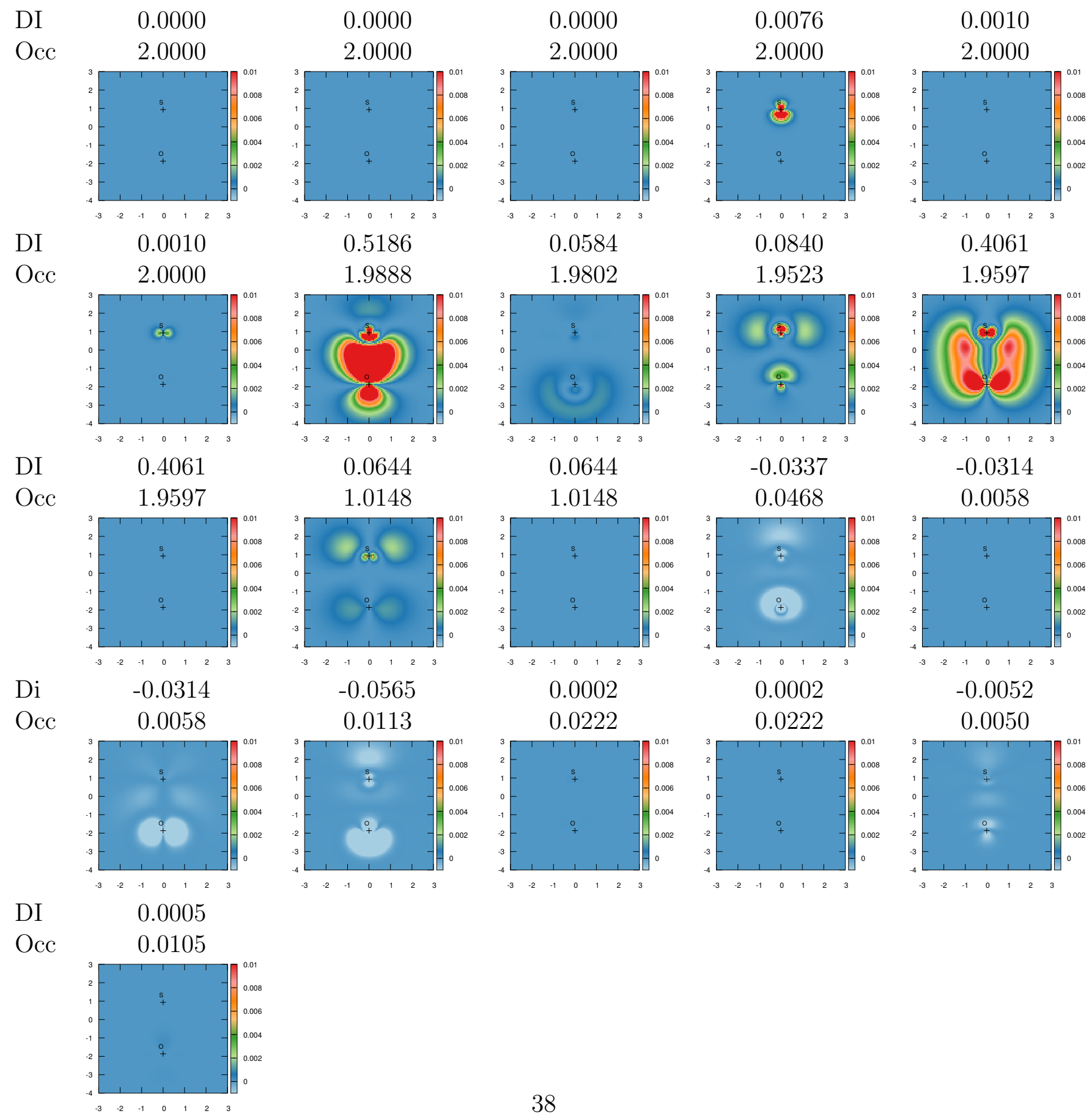


Table S 37: Bonding channels and the corresponding occupation numbers as well as maps of bonding densities for $\mathrm{CN}^{-}$.

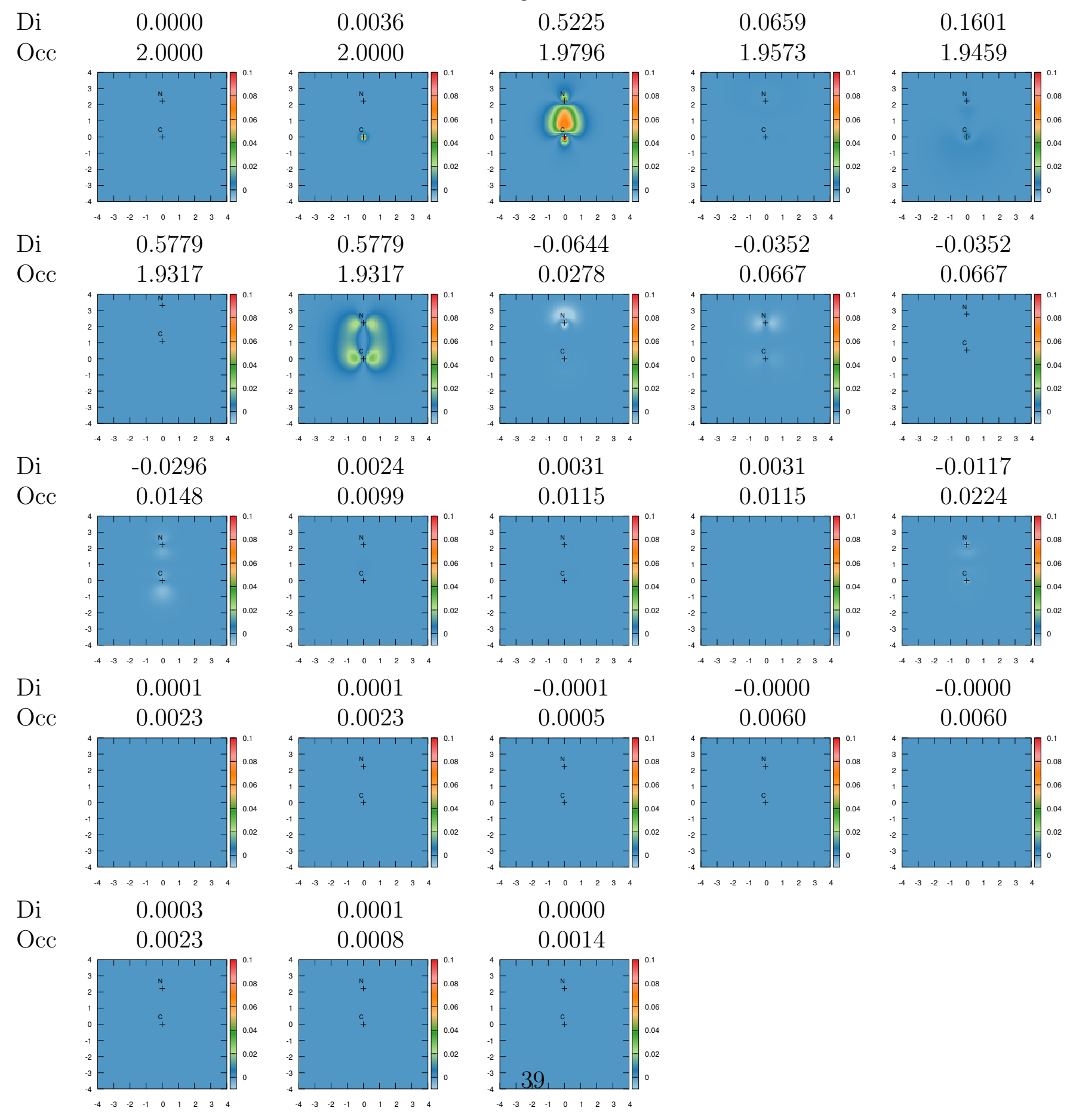


Table S 38: Bonding channels and the corresponding occupation numbers as well as maps of bonding densities for AsN.

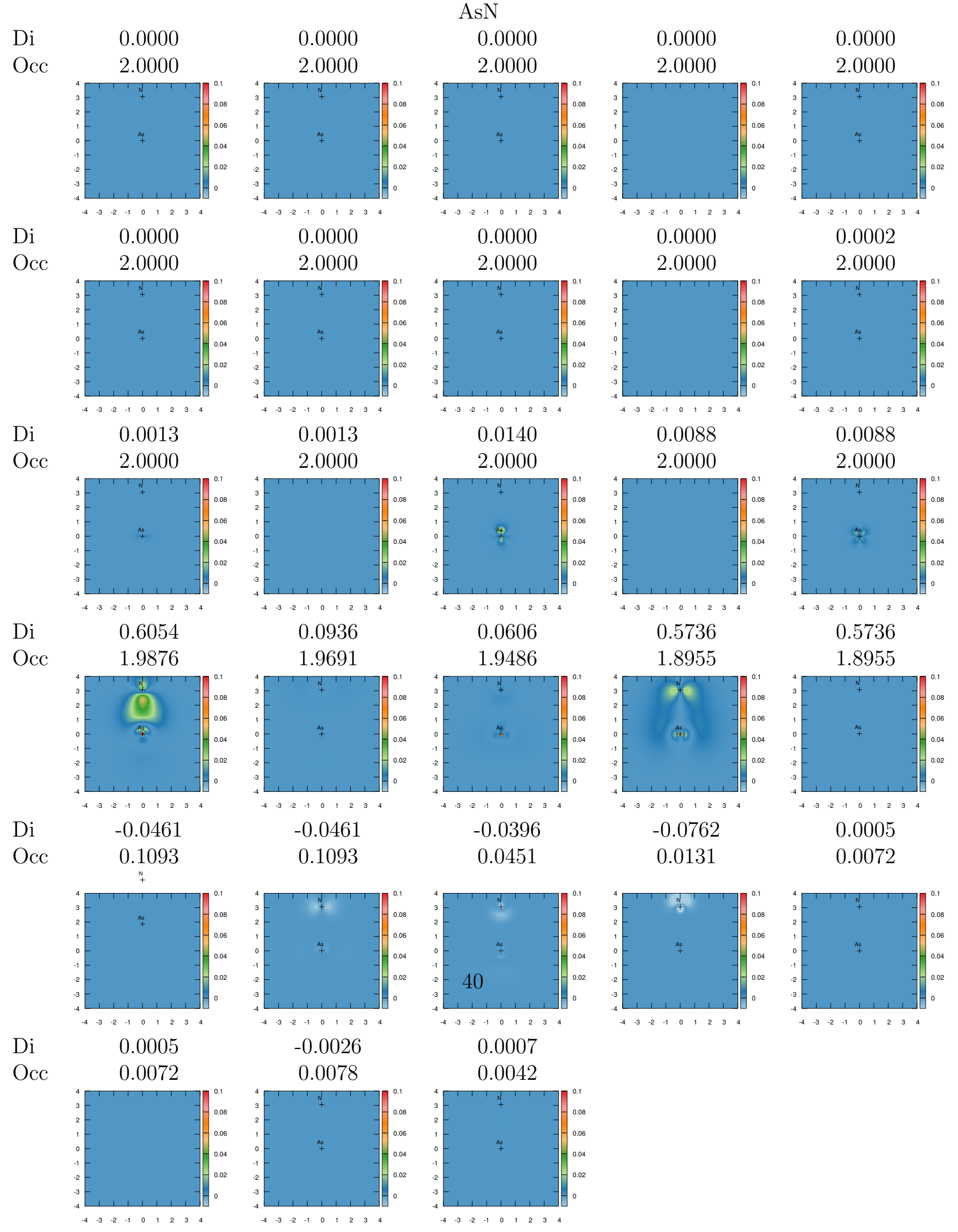


Table S 39: Bonding channels and the corresponding occupation numbers as well as maps of bonding densities for $\mathrm{BC}^{-}$.

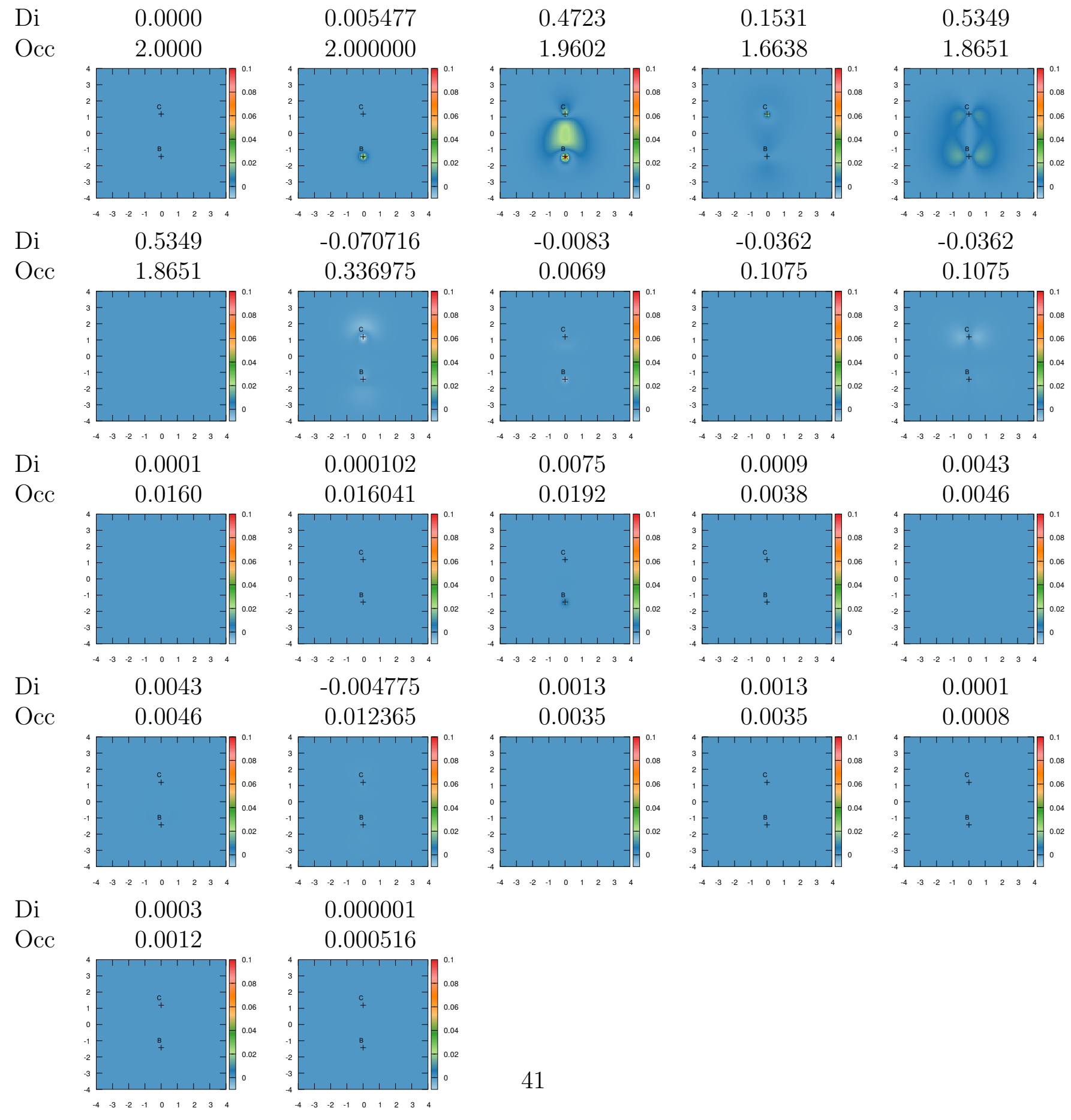


Table S 40: Bonding channels and the corresponding occupation numbers as well as maps of bonding densities for PN.

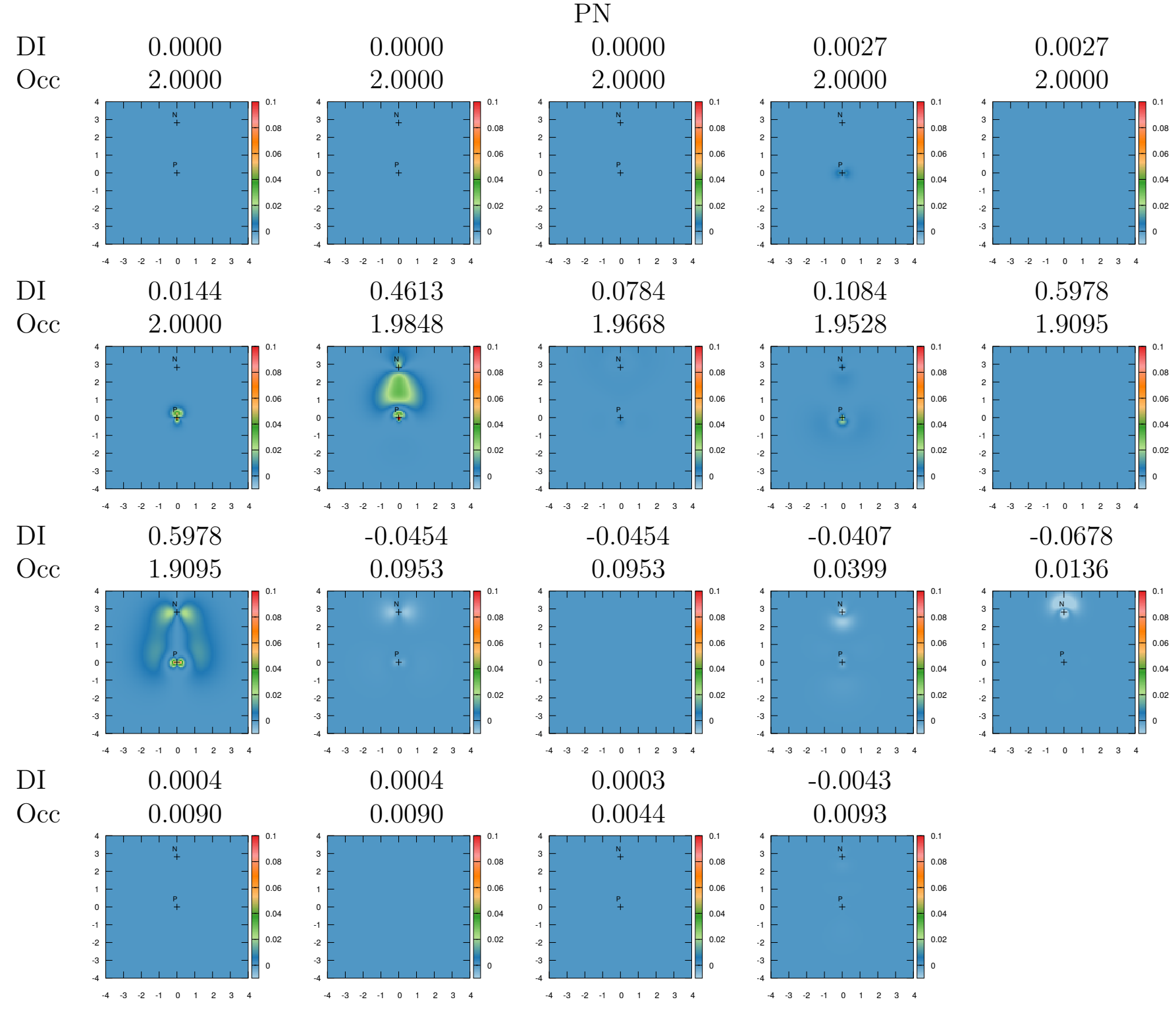


Table S 41: Bonding channels and the corresponding occupation numbers as well as maps of bonding densities for $\mathrm{As}_{2}$, part one.

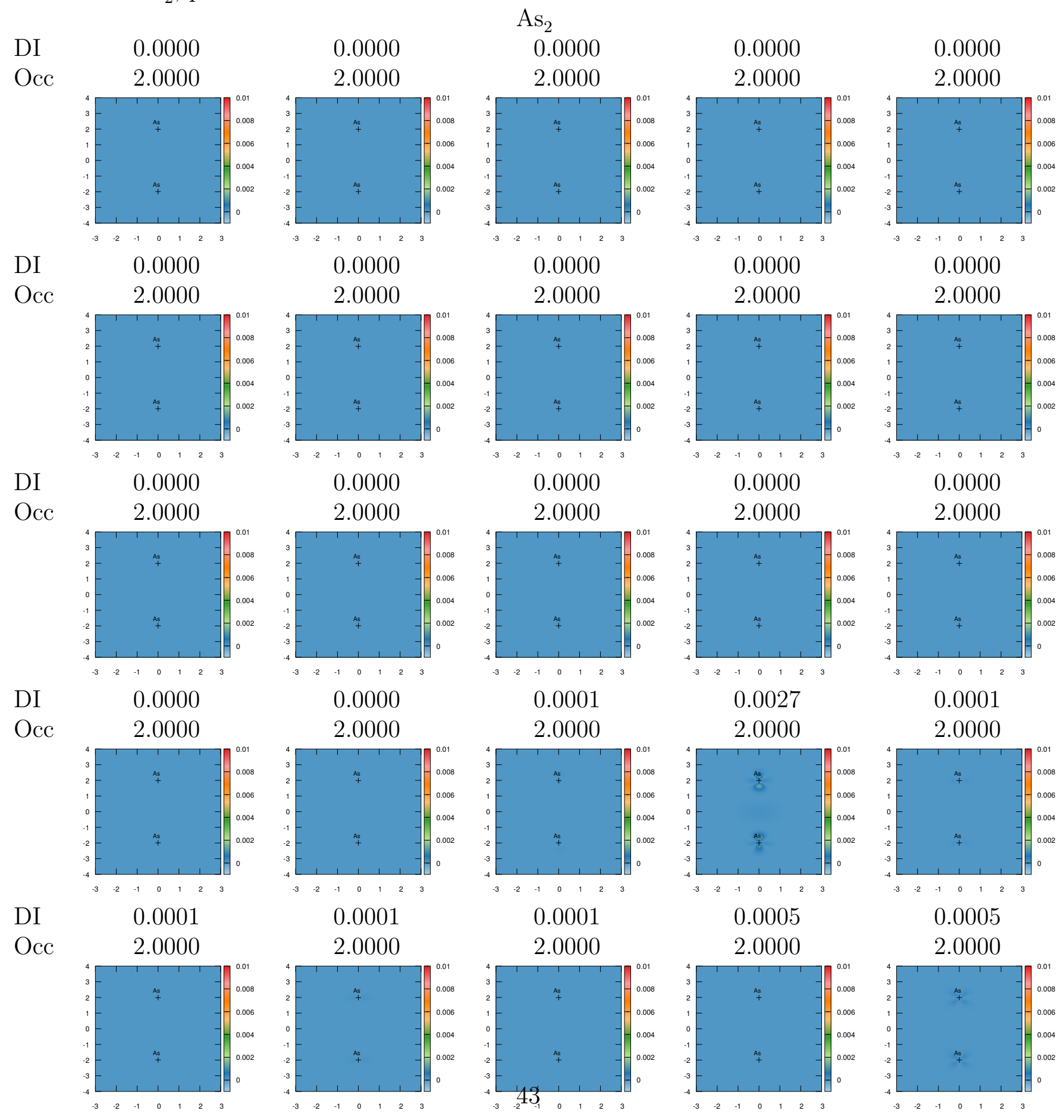


Table S 42: Bonding channels and the corresponding occupation numbers as well as maps of bonding densities for $\mathrm{As}_{2}$, part two.

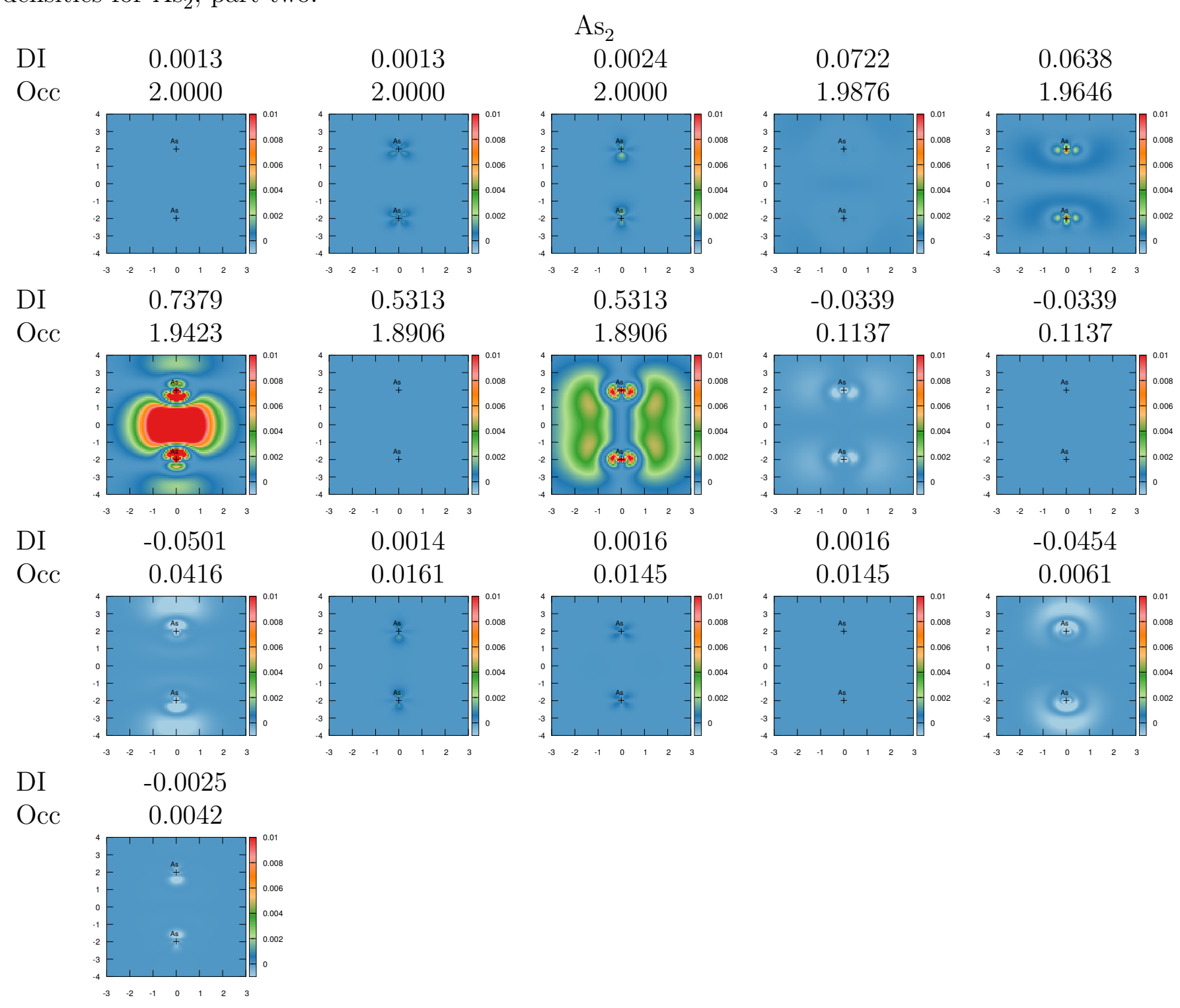


Table S 43: Bonding channels and the corresponding occupation numbers as well as maps of bonding densities for $\mathrm{B}_{2}$.

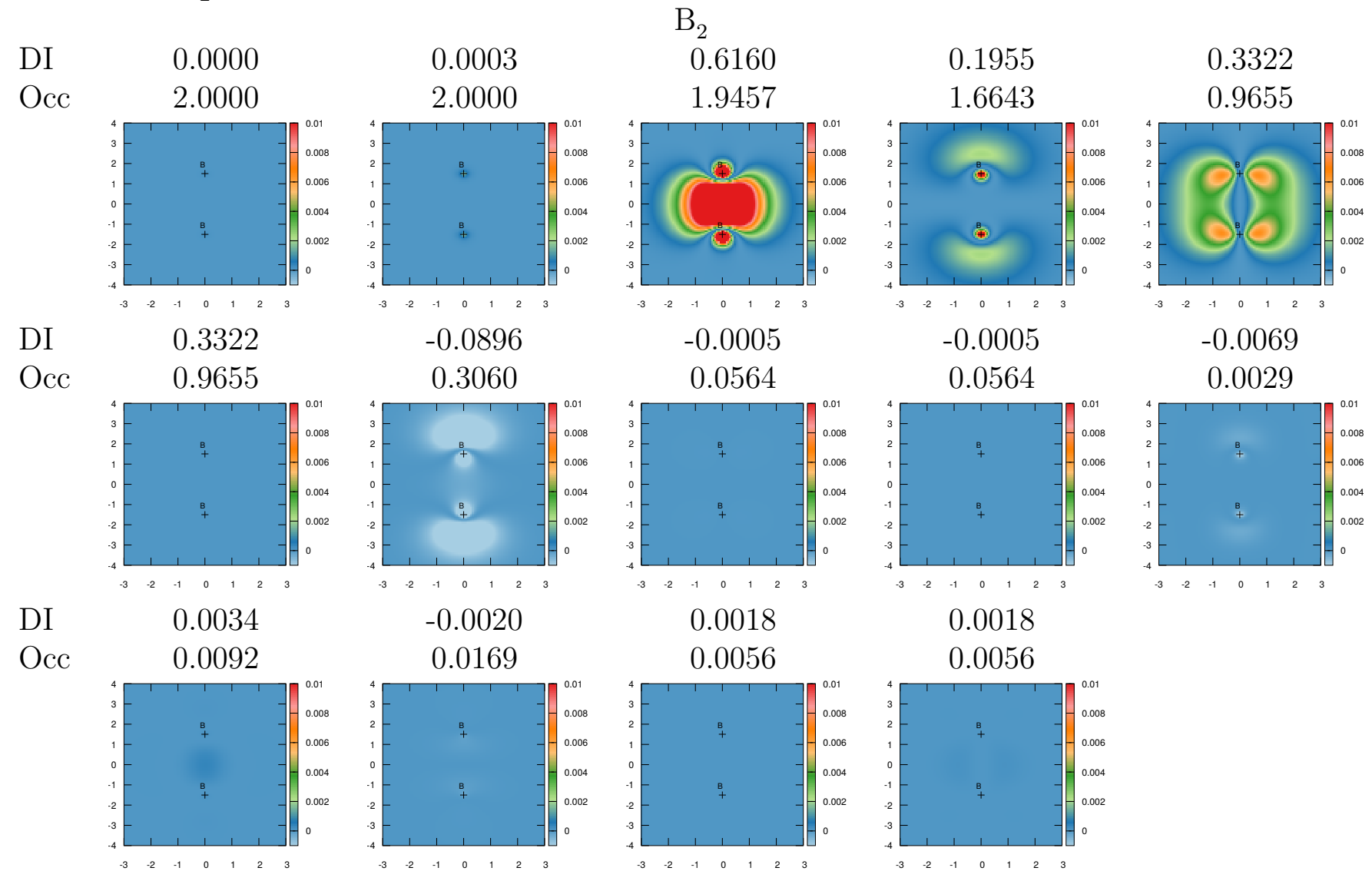


Table S 44: Bonding channels and the corresponding occupation numbers as well as maps of bonding densities for $\mathrm{B}_{2}{ }^{2-}$.

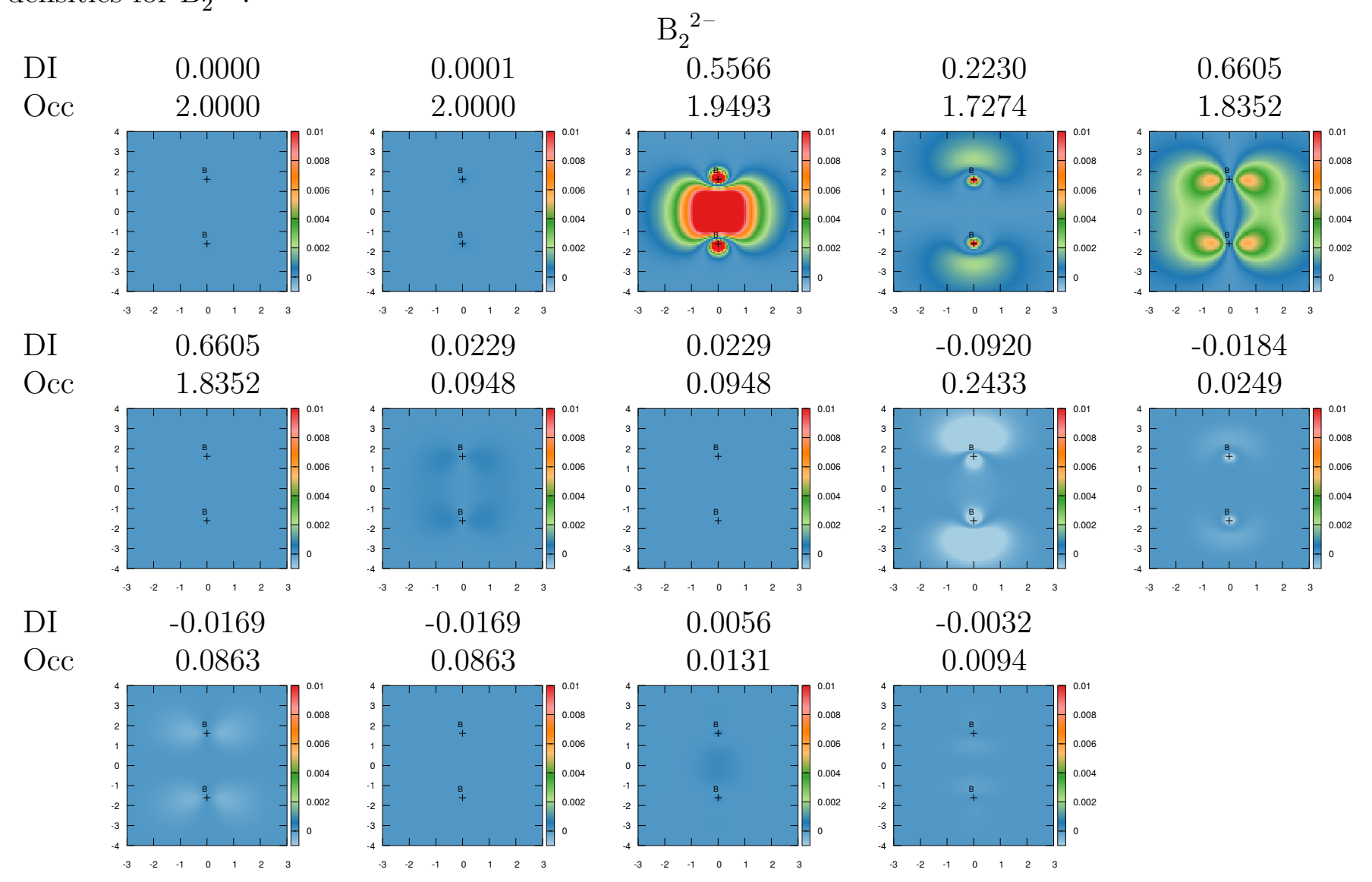


Table S 45: Bonding channels and the corresponding occupation numbers as well as maps of bonding densities for $\mathrm{Be}_{2}$.

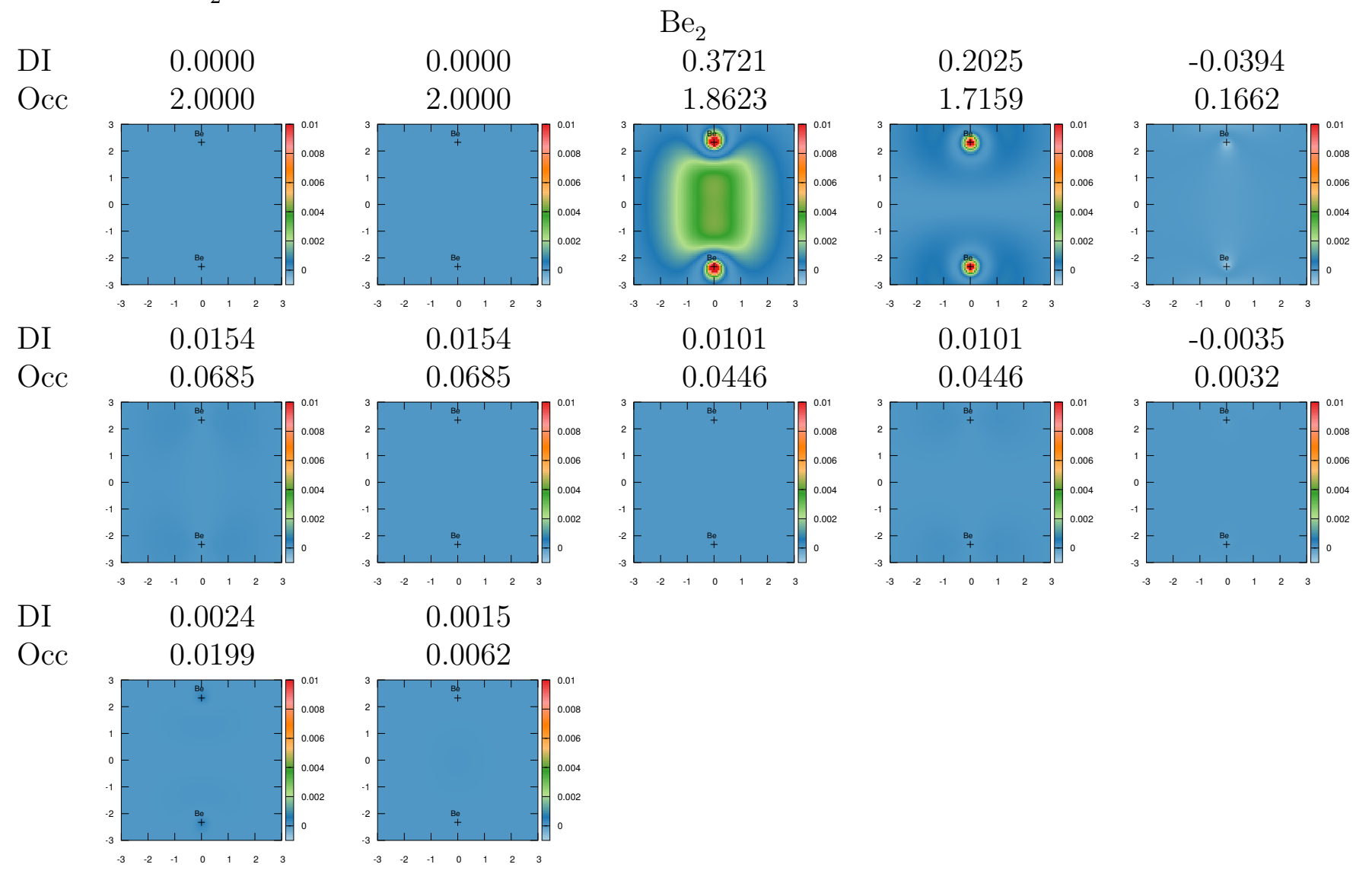


Table S 46: Bonding channels and the corresponding occupation numbers as well as maps of bonding densities for $\mathrm{Br}_{2}$, par one.

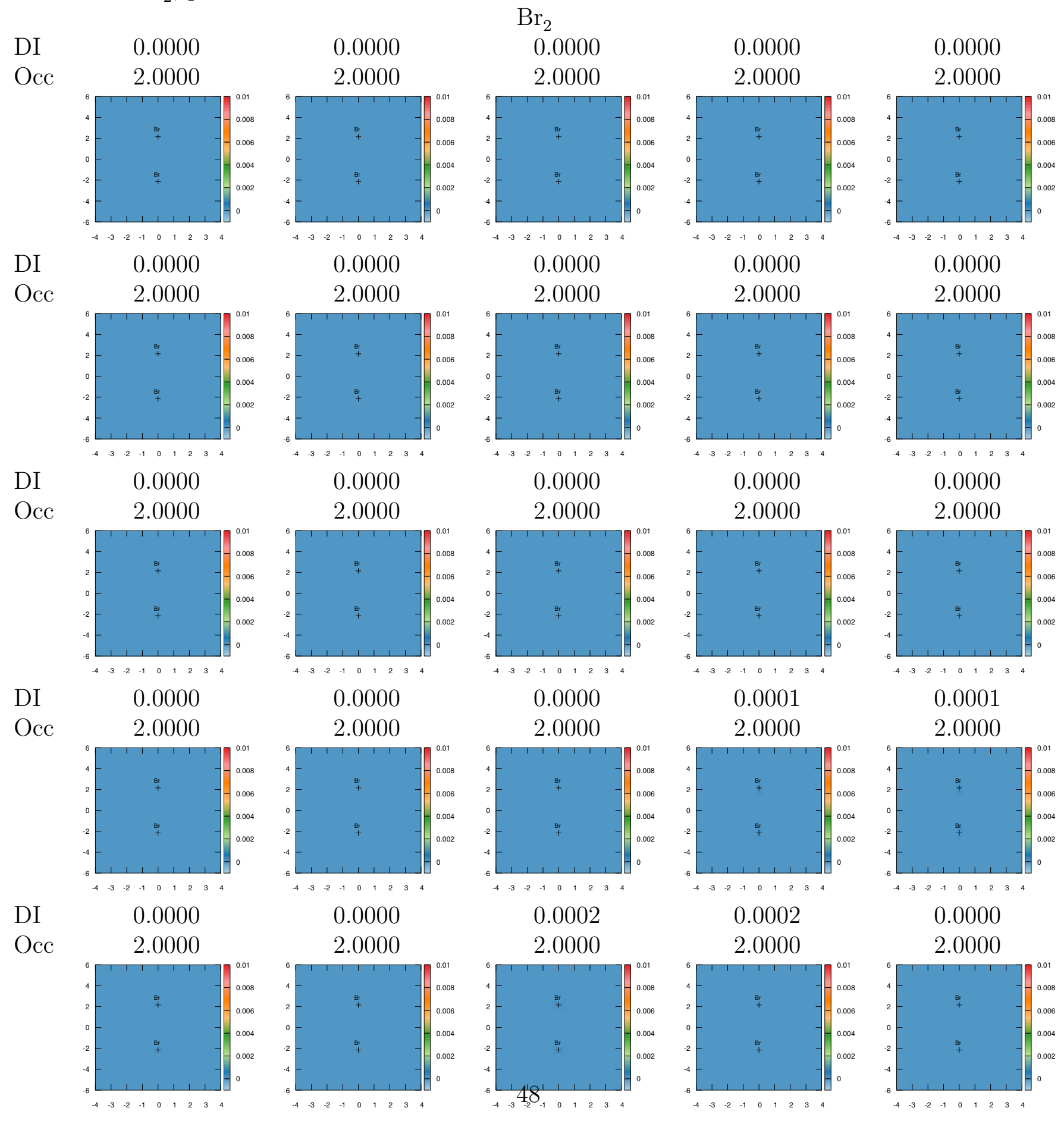


Table S 47: Bonding channels and the corresponding occupation numbers as well as maps of bonding densities for $\mathrm{Br}_{2}$, par two.

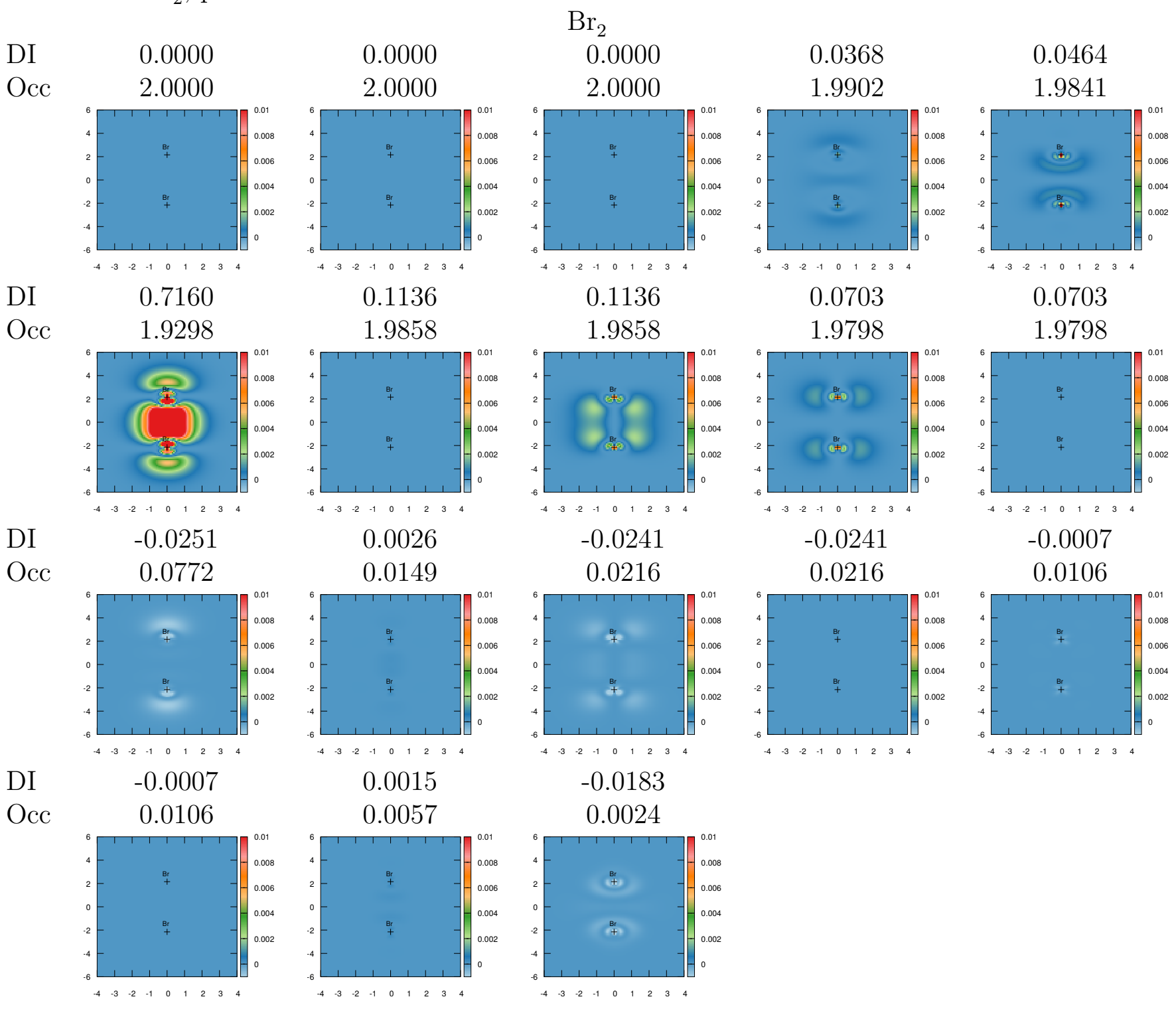


Table S 48: Bonding channels and the corresponding occupation numbers as well as maps of bonding densities for $\mathrm{C}_{2}$.

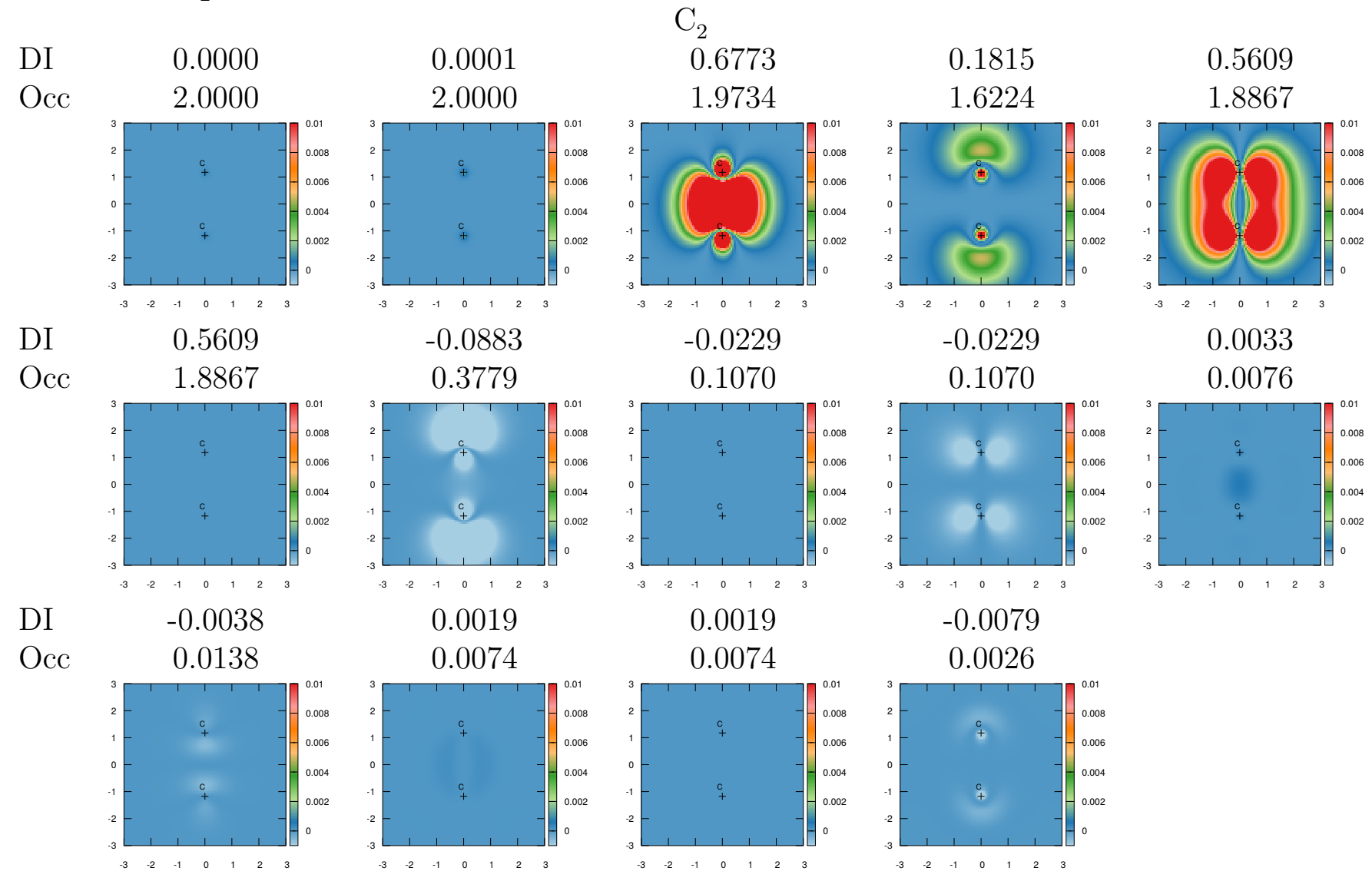


Table S 49: Bonding channels and the corresponding occupation numbers as well as maps of bonding densities for $\mathrm{C}_{2}{ }^{2-}$.

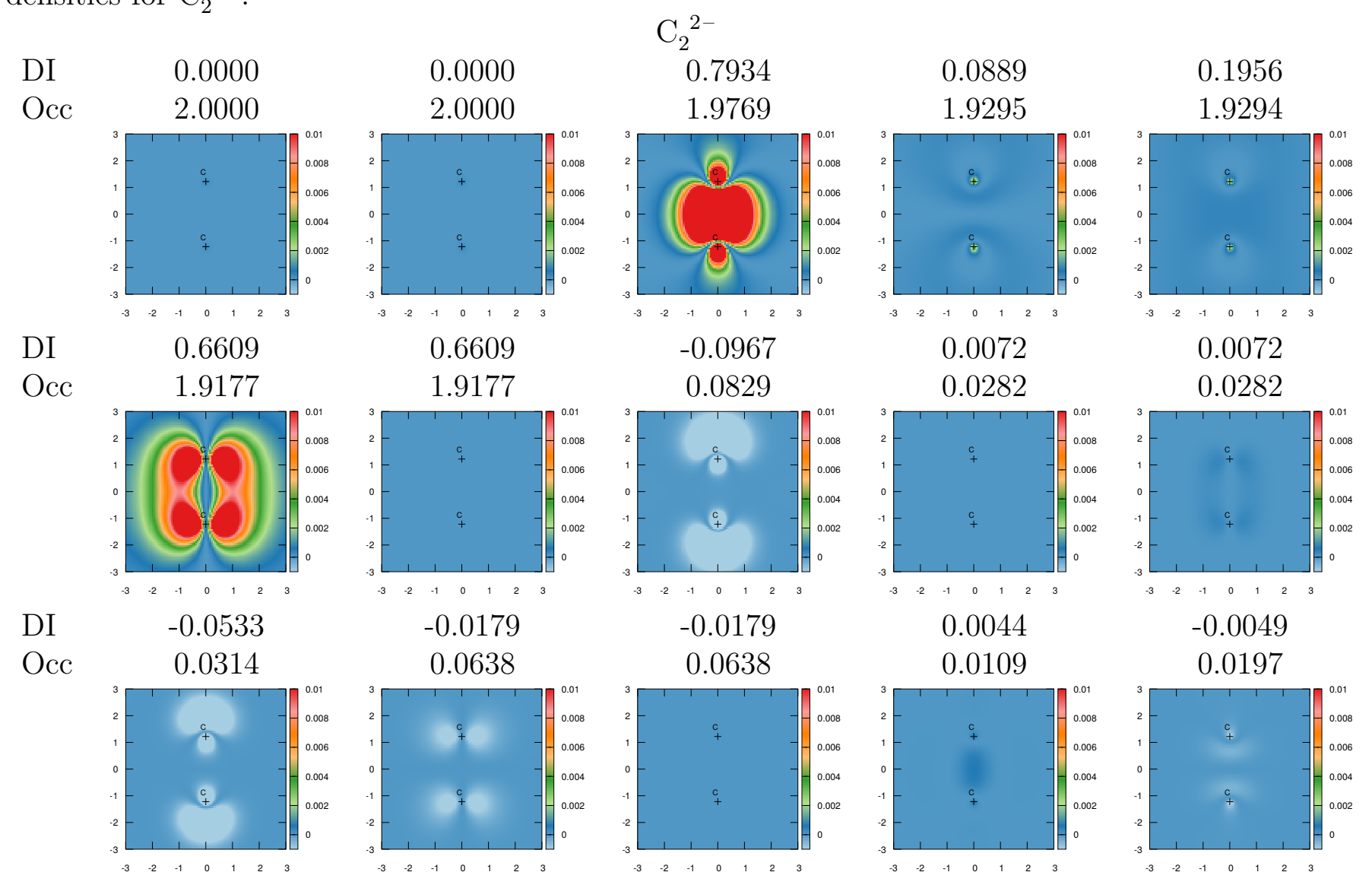


Table S 50: Bonding channels and the corresponding occupation numbers as well as maps of bonding densities for $\mathrm{Ca}_{2}$.

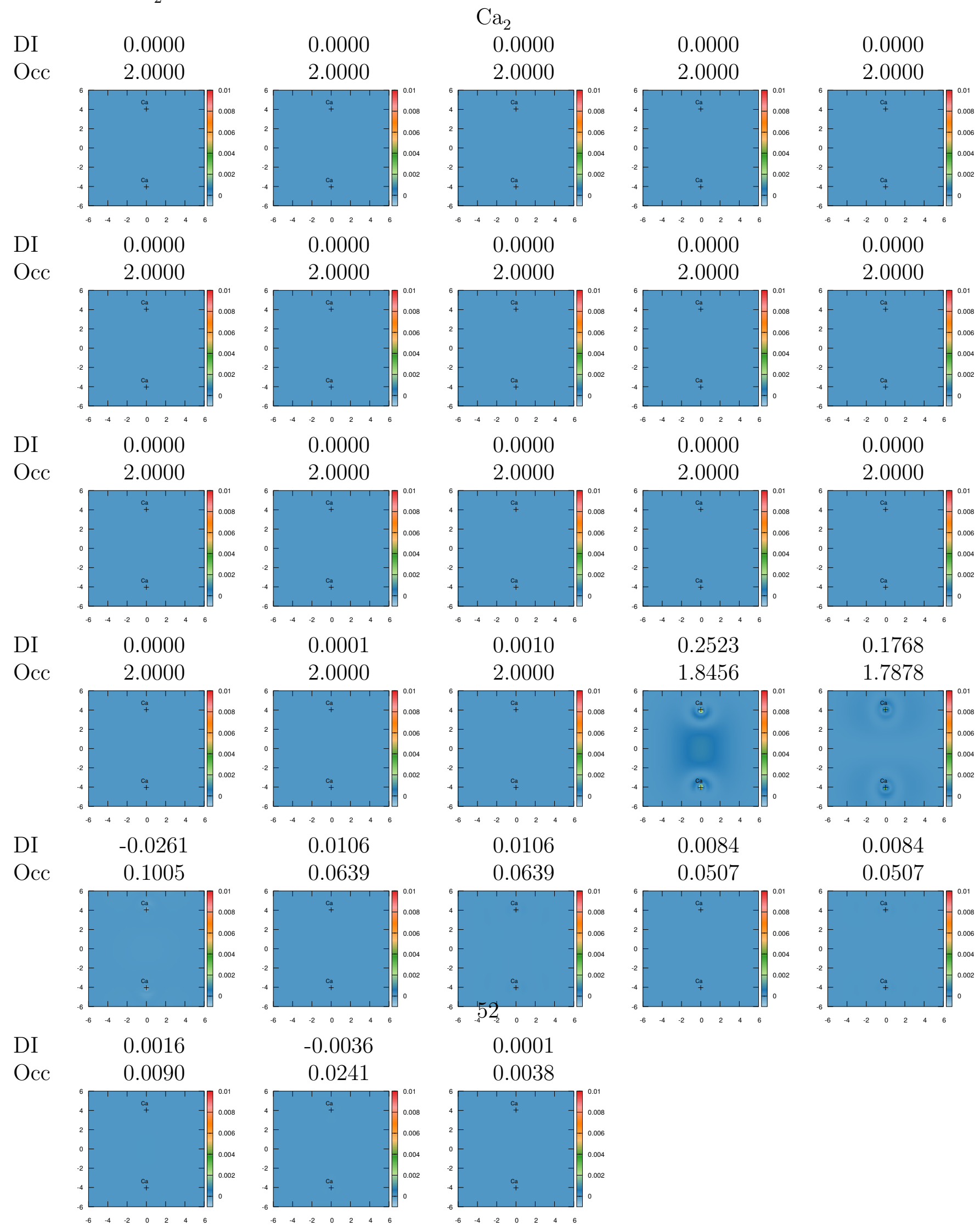


Table S 51: Bonding channels and the corresponding occupation numbers as well as maps of bonding densities for $\mathrm{Cl}_{2}$.

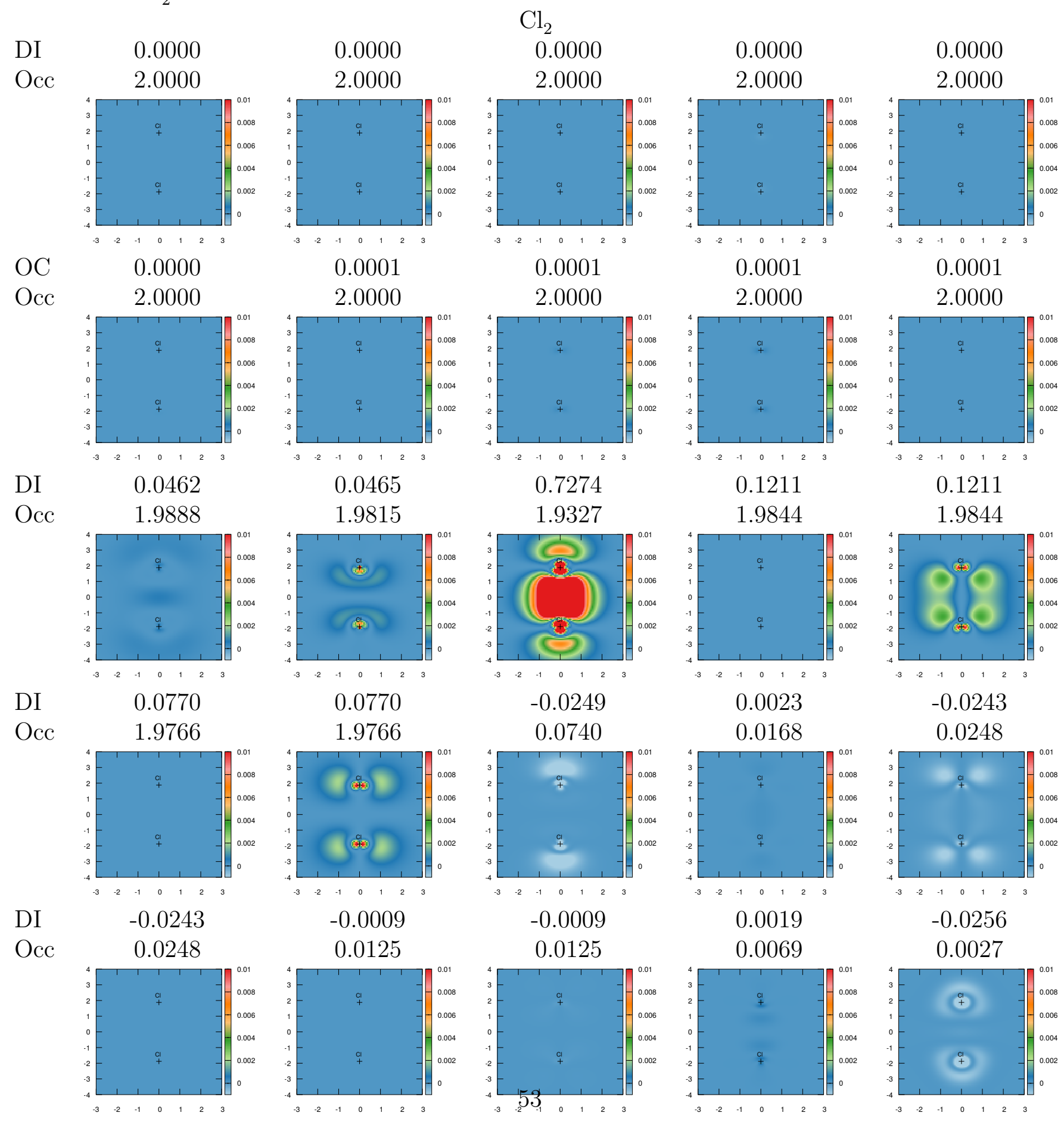


Table S 52: Bonding channels and the corresponding occupation numbers as well as maps of bonding densities for $\mathrm{F}_{2}$.

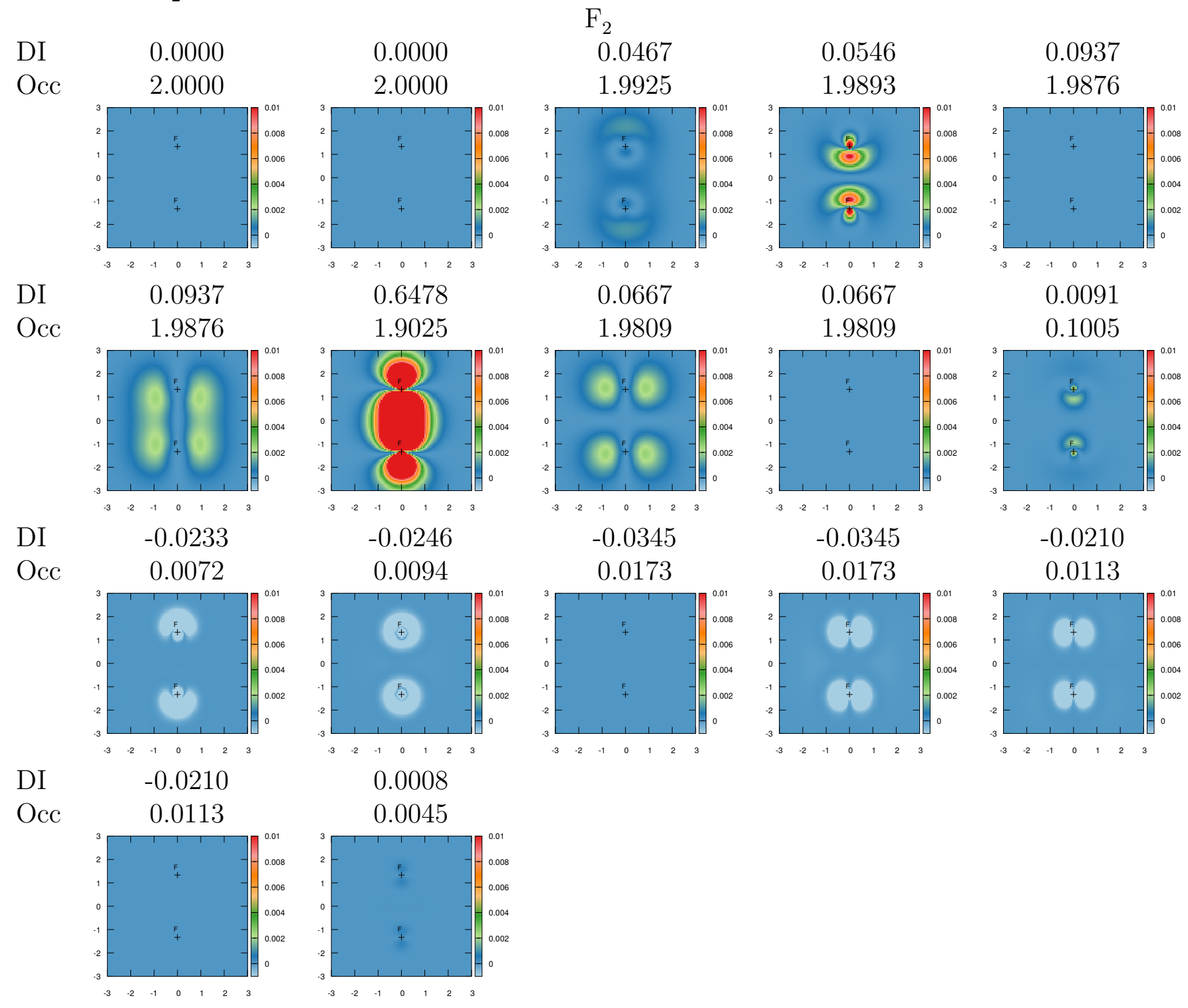


Table S 53: Bonding channels and the corresponding occupation numbers as well as maps of bonding densities for $\mathrm{Ge}_{2}$, part one.

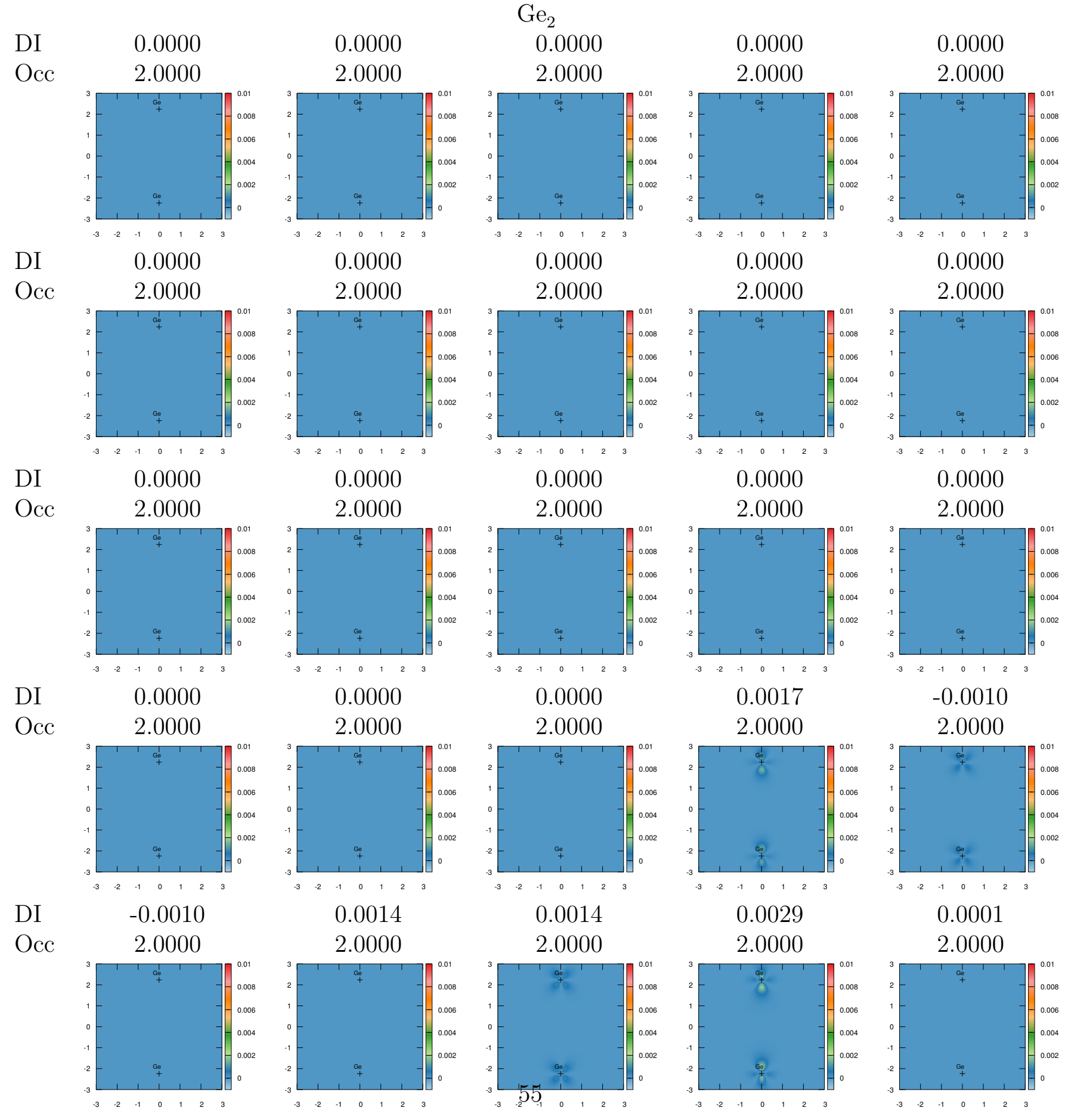


Table S 54: Bonding channels and the corresponding occupation numbers as well as maps of bonding densities for $\mathrm{Ge}_{2}$, part two.

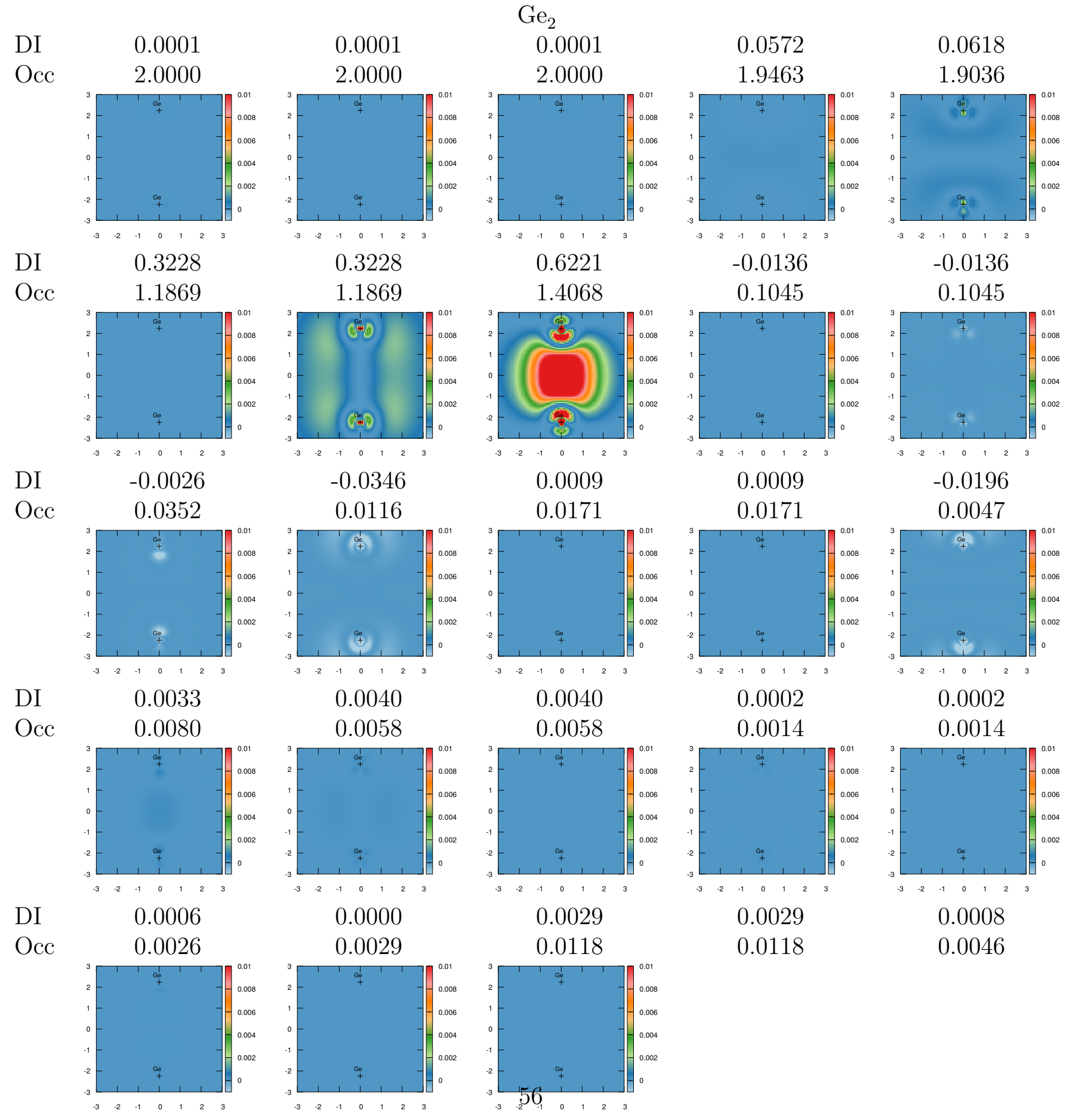


Table S 55: Bonding channels and the corresponding occupation numbers as well as maps of bonding densities for $\mathrm{H}_{2}$.

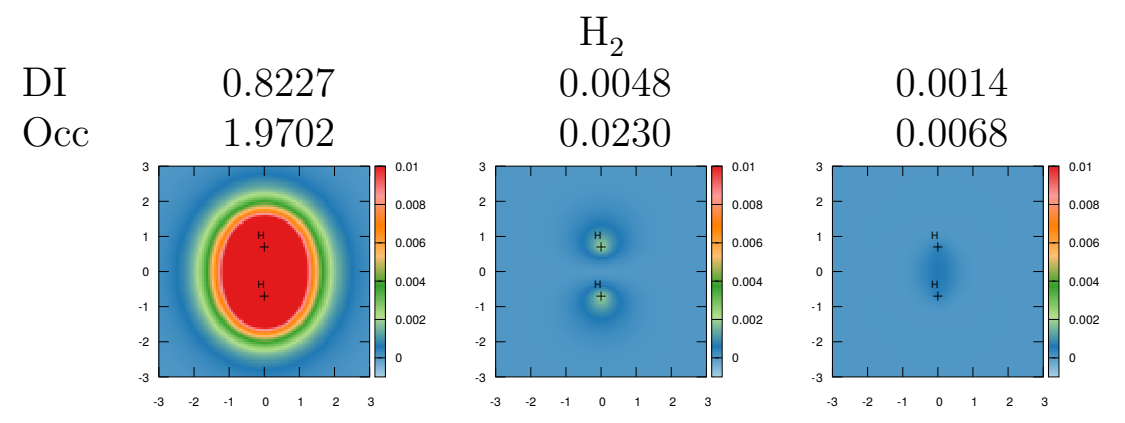


Table S 56: Bonding channels and the corresponding occupation numbers as well as maps of bonding densities for $\mathrm{K}_{2}$.

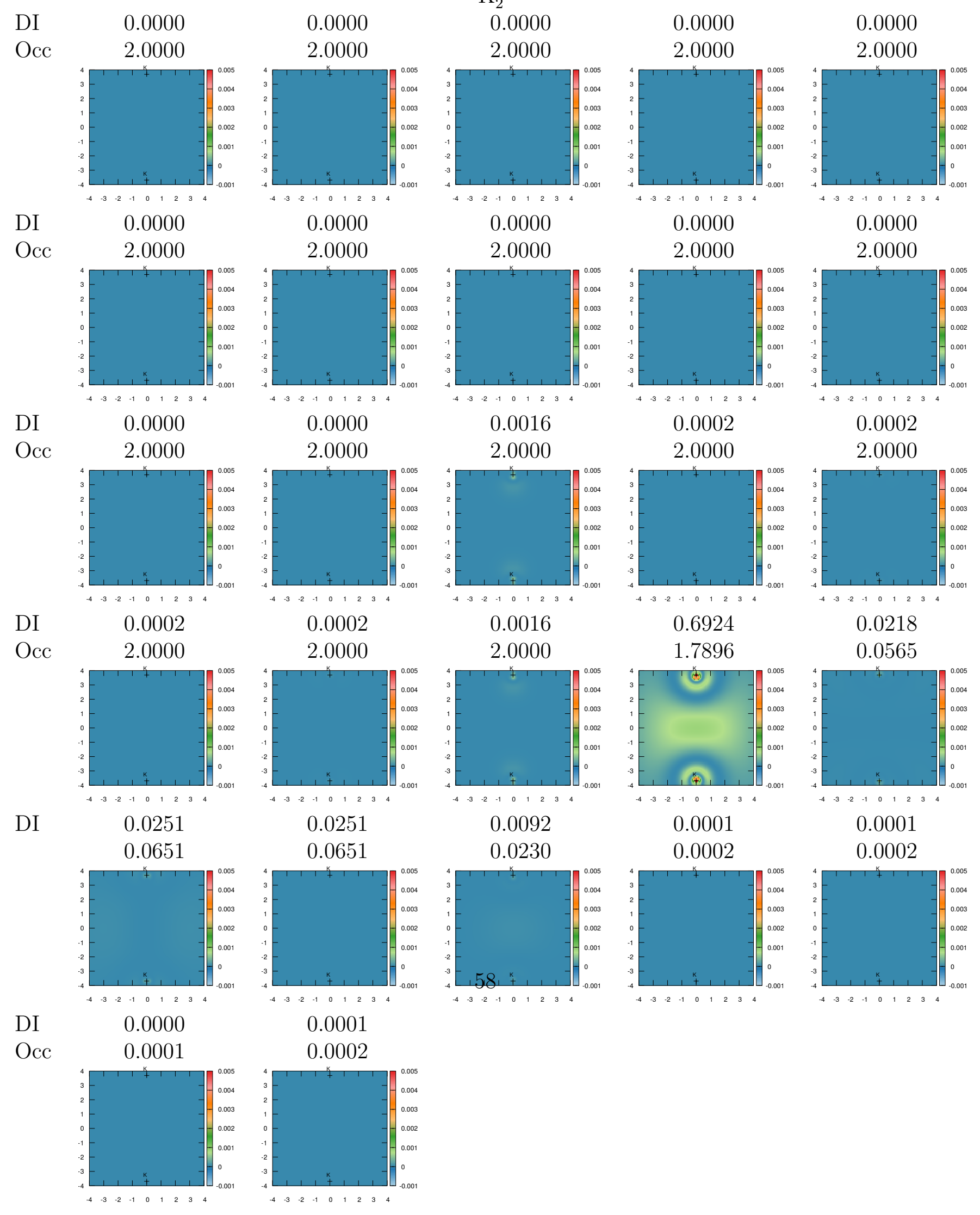


Table S 57: Bonding channels and the corresponding occupation numbers as well as maps of bonding densities for $\mathrm{Li}_{2}$.

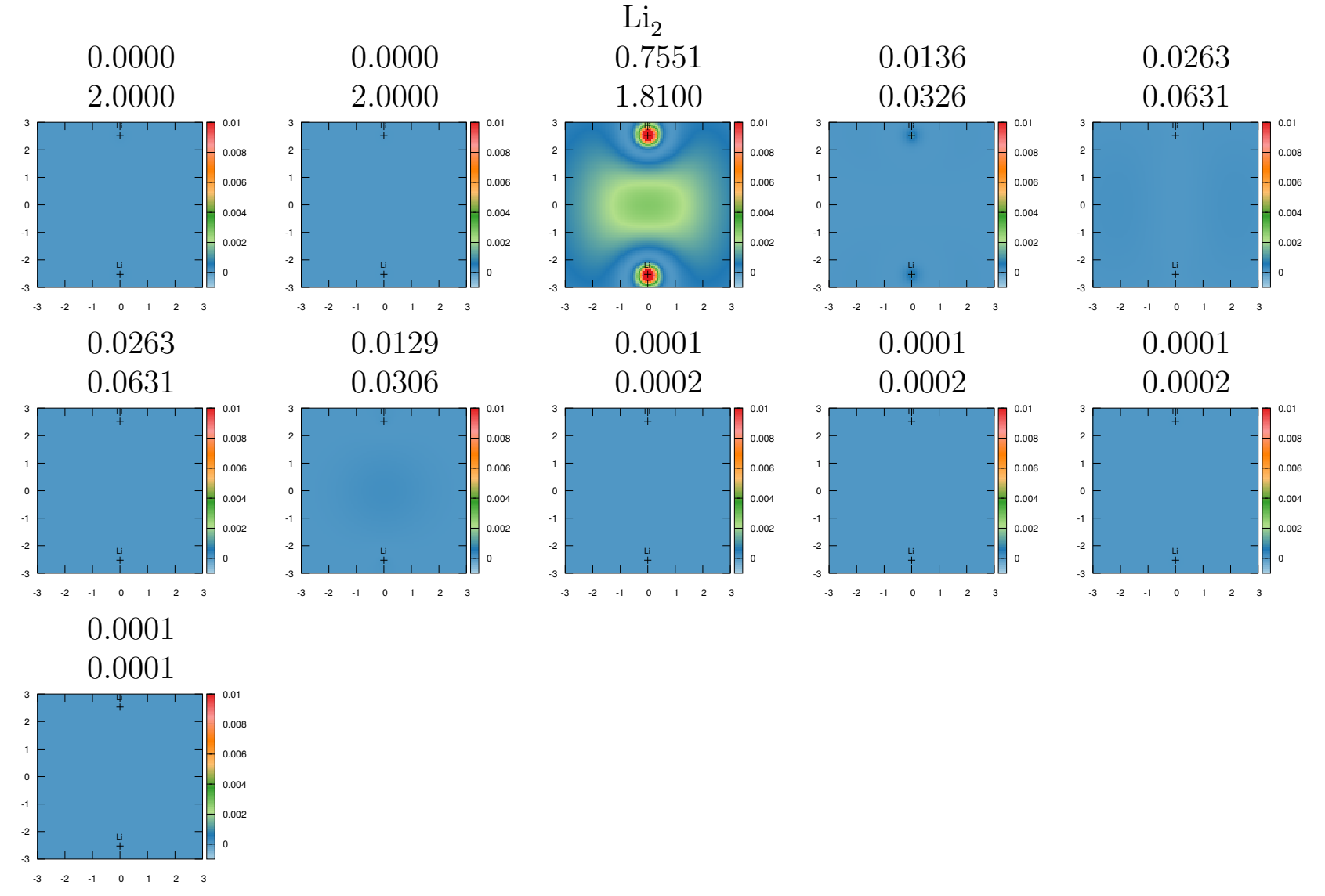


Table S 58: Bonding channels and the corresponding occupation numbers as well as maps of bonding densities for $\mathrm{Mg}_{2}$.

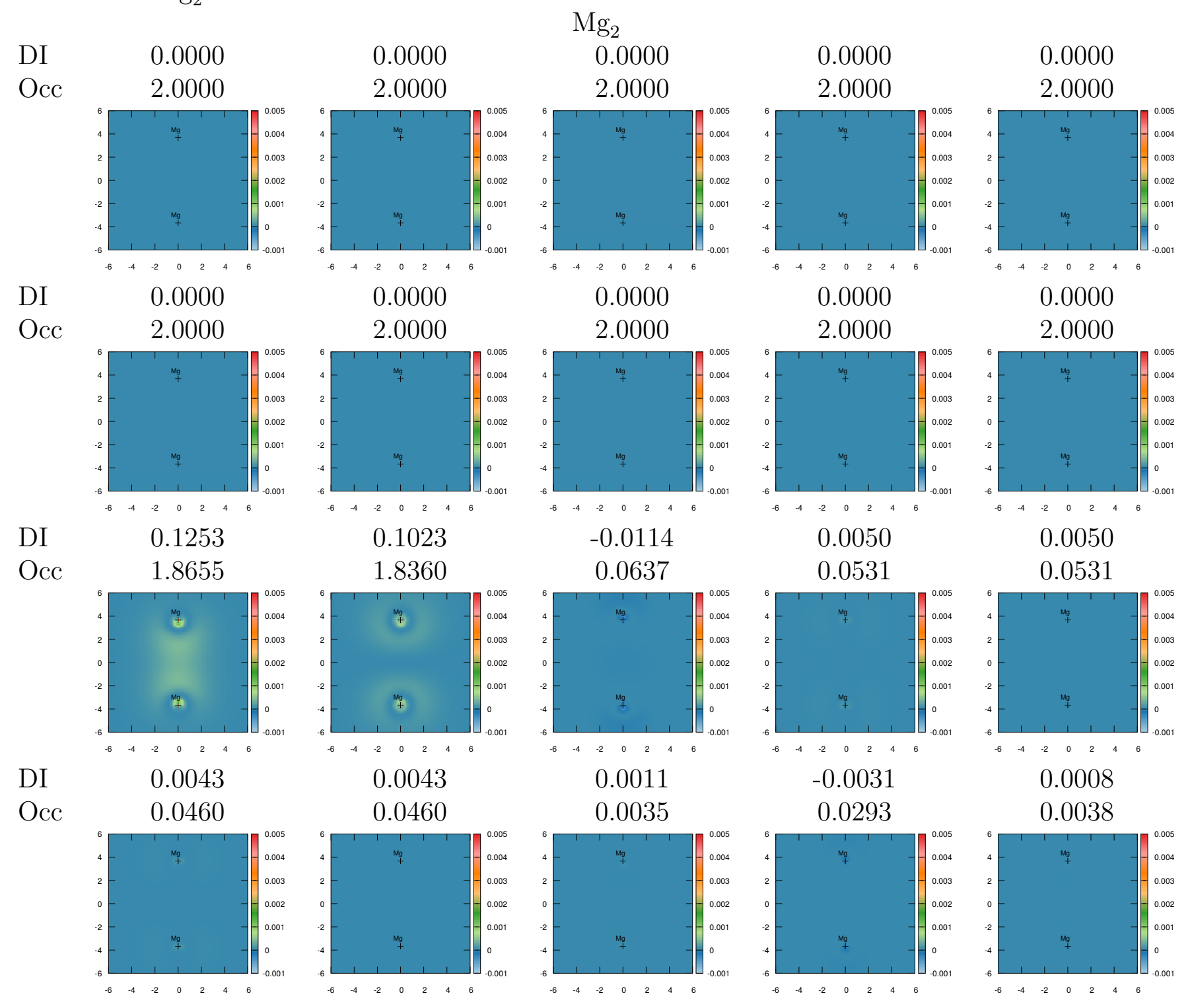


Table S 59: Bonding channels and the corresponding occupation numbers as well as maps of bonding densities for $\mathrm{N}_{2}$.

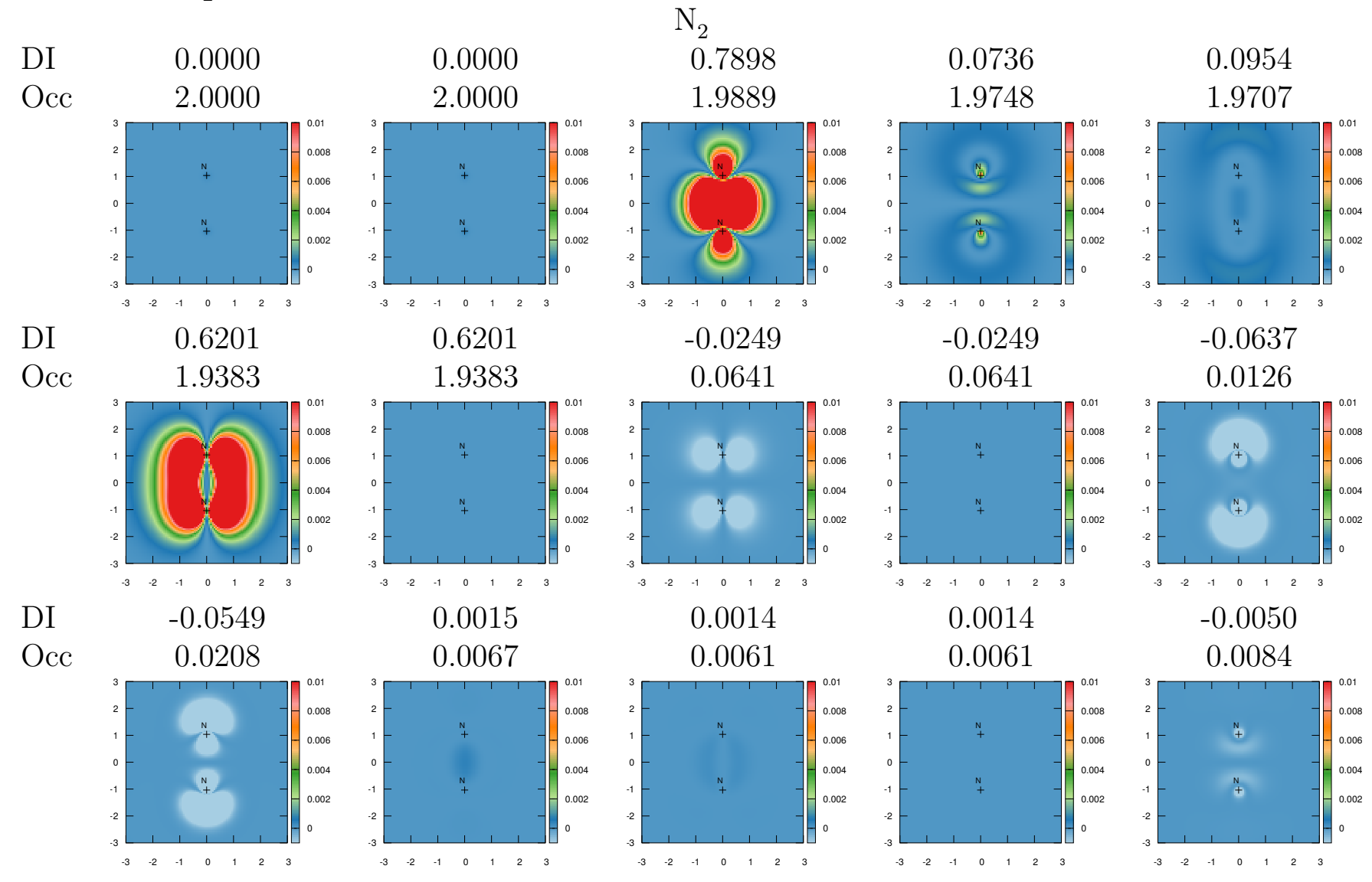


Table S 60: Bonding channels and the corresponding occupation numbers as well as maps of bonding densities for $\mathrm{Na}_{2}$.

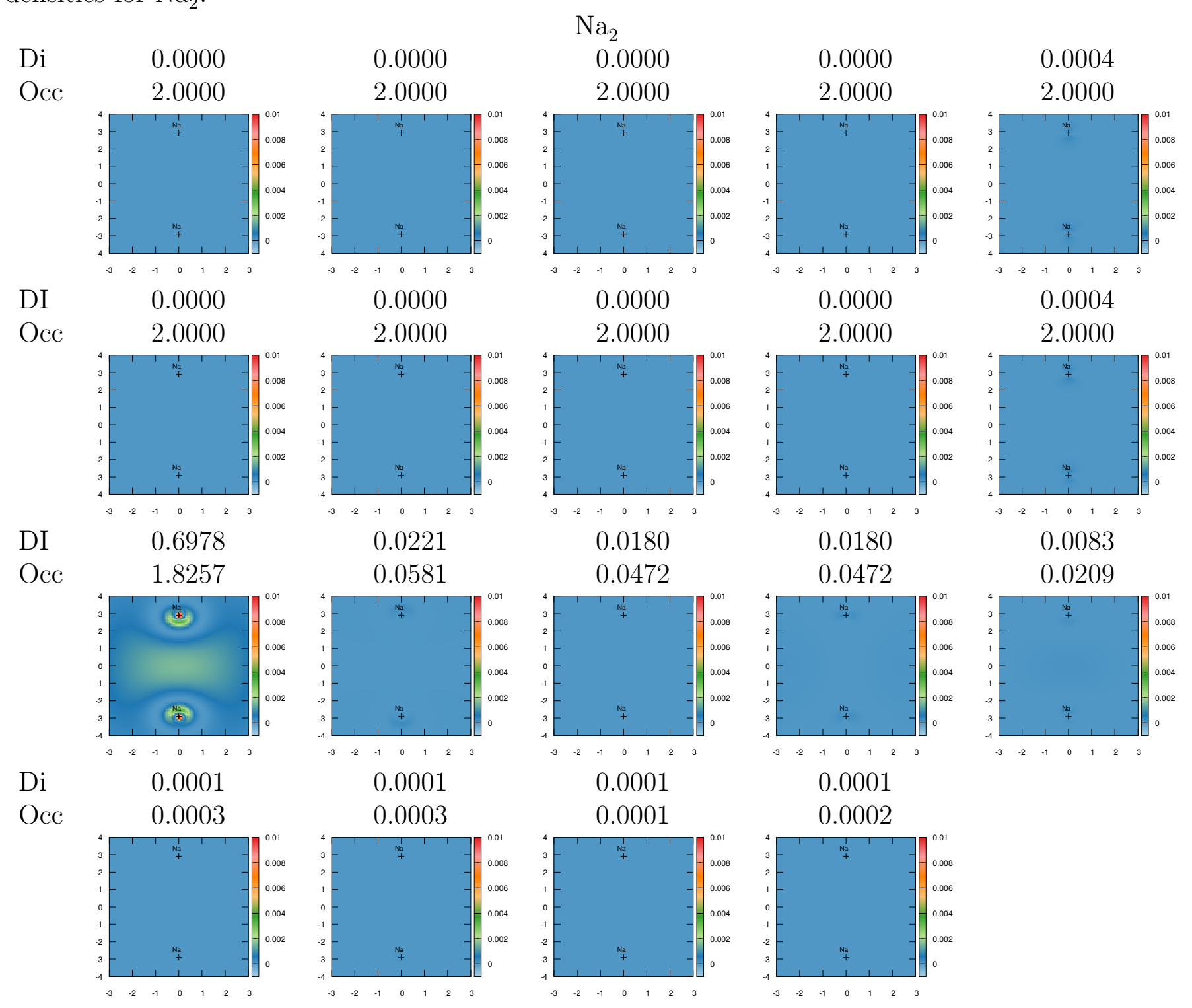


Table S 61: Bonding channels and the corresponding occupation numbers as well as maps of bonding densities for $\mathrm{O}_{2}$.

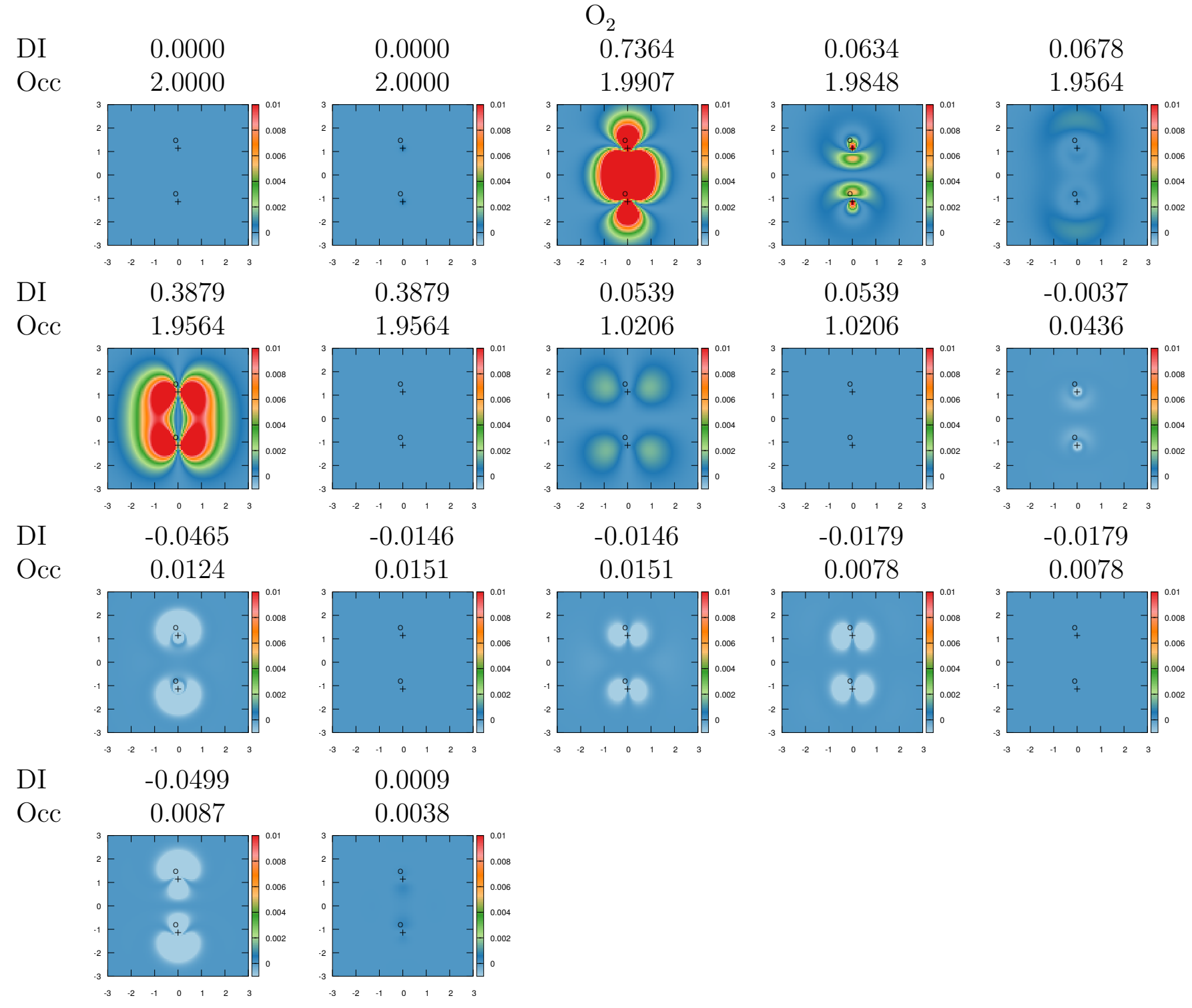


Table S 62: Bonding channels and the corresponding occupation numbers as well as maps of bonding densities for $\mathrm{P}_{2}$.

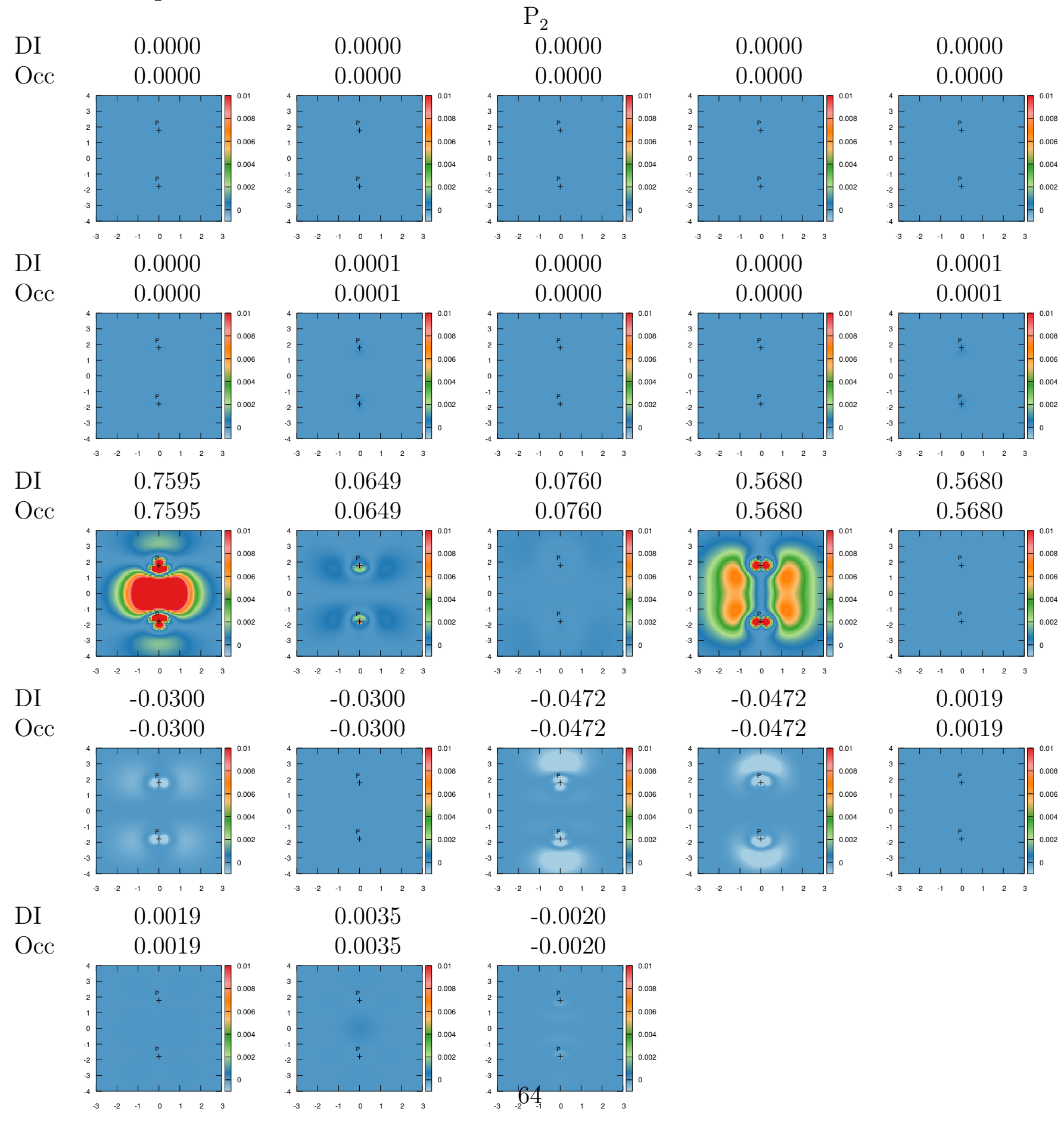


Table S 63: Bonding channels and the corresponding occupation numbers as well as maps of bonding densities for $\mathrm{Se}_{2}$, part one.

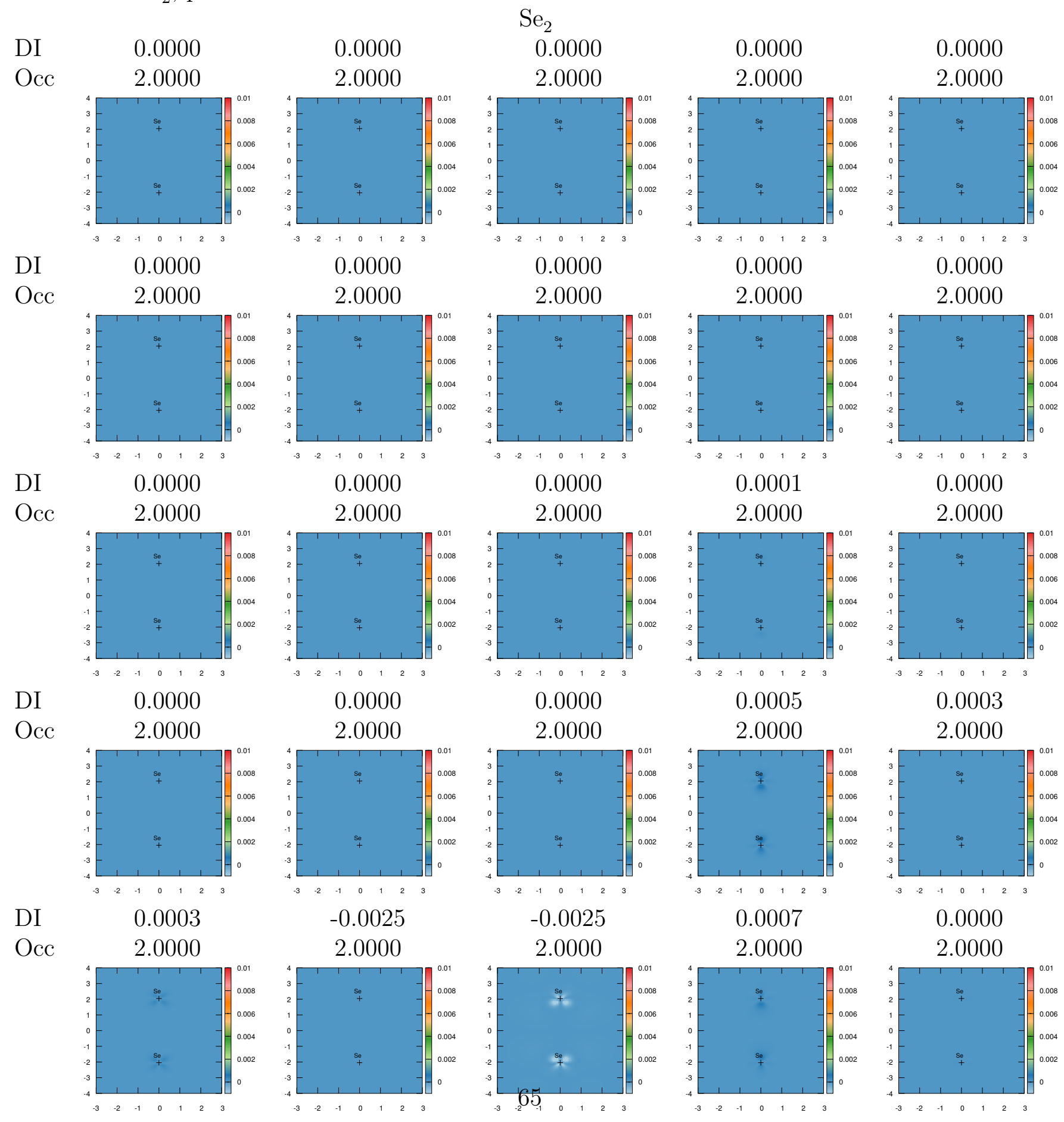


Table S 64: Bonding channels and the corresponding occupation numbers as well as maps of bonding densities for $\mathrm{Se}_{2}$, part two.

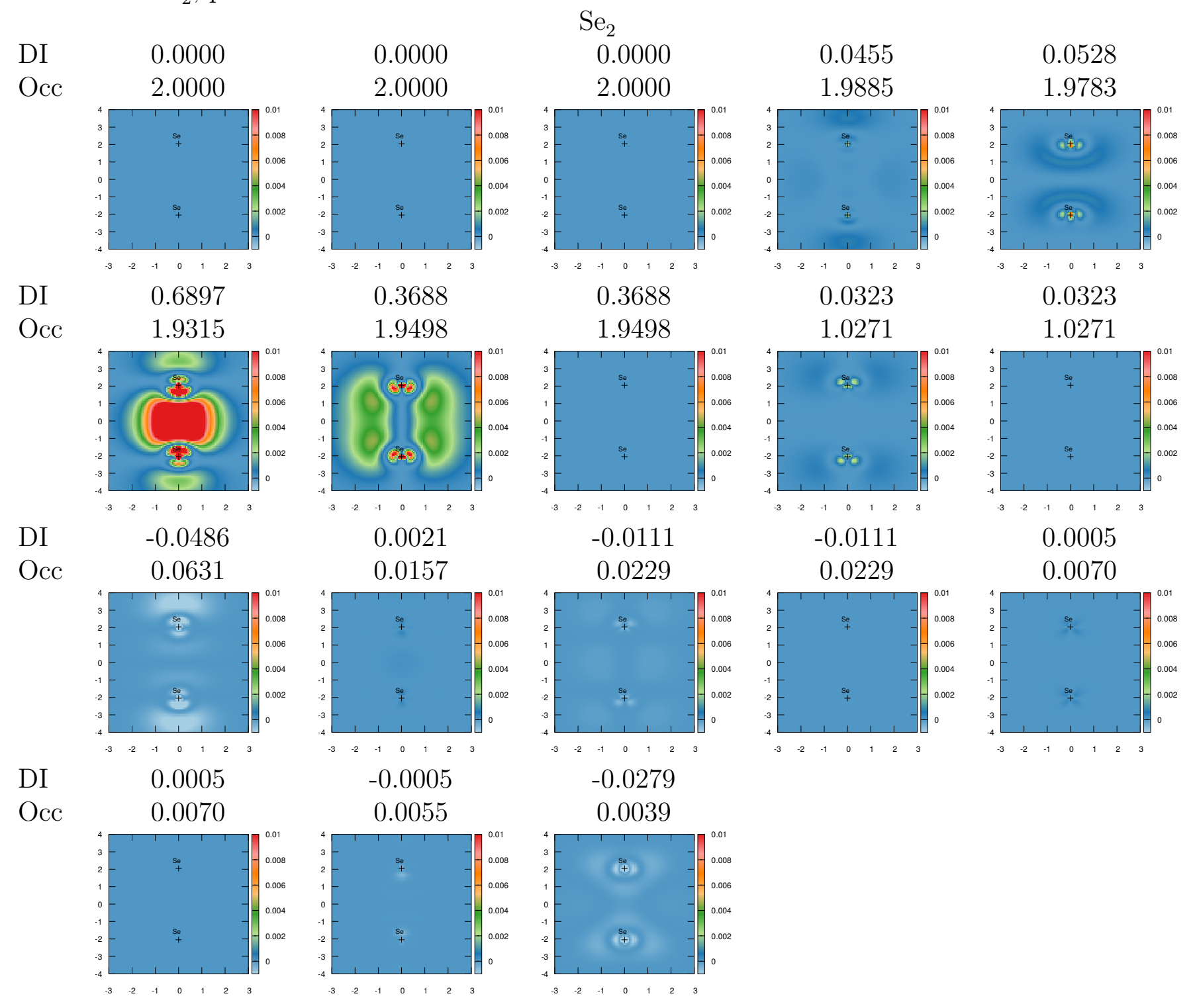


Table S 65: Bonding channels and the corresponding occupation numbers as well as maps of bonding densities for $\mathrm{Si}_{2}$.

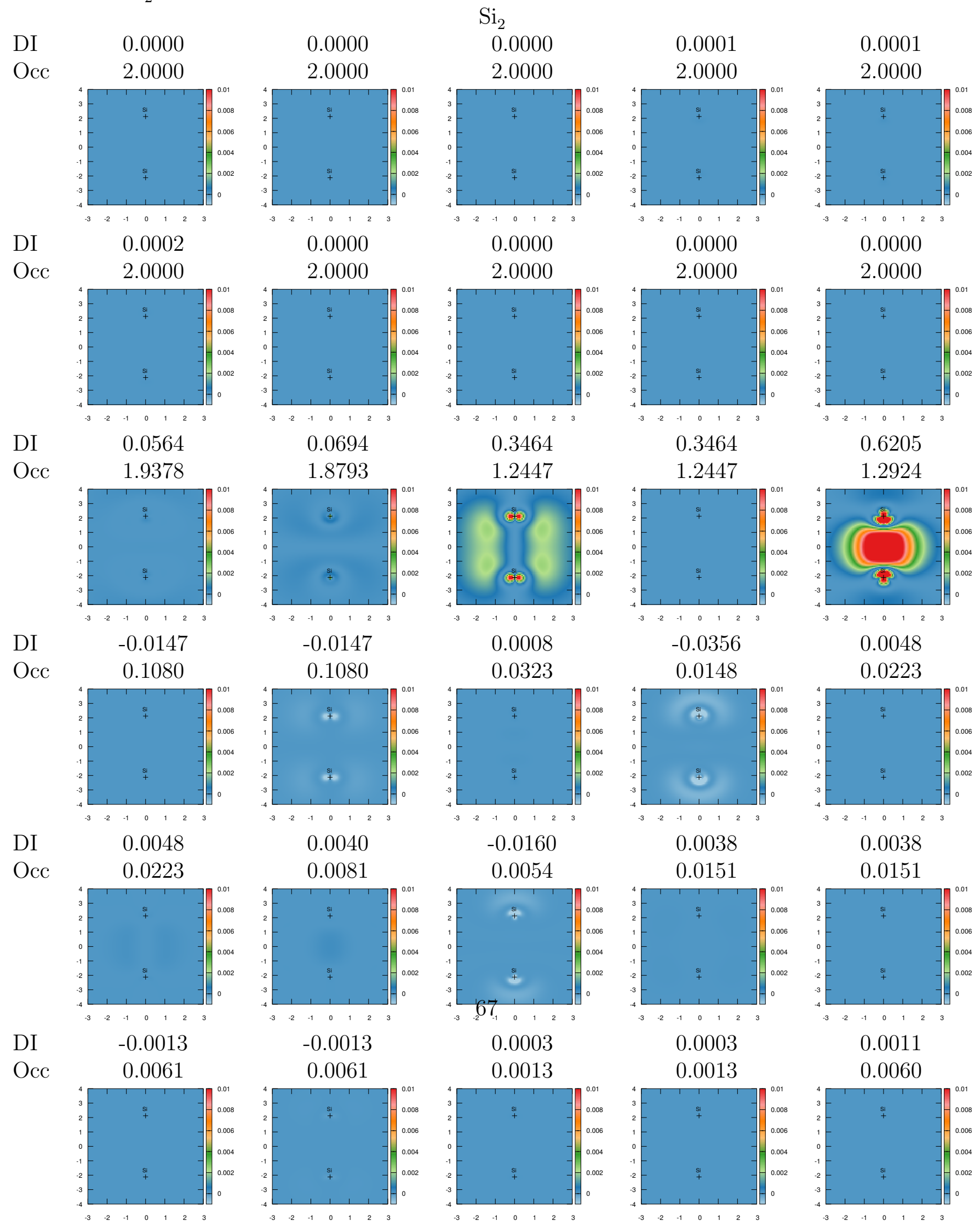

